\title{
Organ saving treatment after chemoradiotherapy for rectal cancer : MRI as a selection tool
}

Citation for published version (APA):

Lambregts, D. M. J. (2013). Organ saving treatment after chemoradiotherapy for rectal cancer : MRI as a selection tool. [Doctoral Thesis, Maastricht University]. Datawyse / Universitaire Pers Maastricht. https://doi.org/10.26481/dis.20130508dl

Document status and date:

Published: 01/01/2013

DOI:

$10.26481 /$ dis.20130508dl

Document Version:

Publisher's PDF, also known as Version of record

\section{Please check the document version of this publication:}

- A submitted manuscript is the version of the article upon submission and before peer-review. There can be important differences between the submitted version and the official published version of record.

People interested in the research are advised to contact the author for the final version of the publication, or visit the DOI to the publisher's website.

- The final author version and the galley proof are versions of the publication after peer review.

- The final published version features the final layout of the paper including the volume, issue and page numbers.

Link to publication

\footnotetext{
General rights rights.

- You may freely distribute the URL identifying the publication in the public portal. please follow below link for the End User Agreement:

www.umlib.nl/taverne-license

Take down policy

If you believe that this document breaches copyright please contact us at:

repository@maastrichtuniversity.nl

providing details and we will investigate your claim.
}

Copyright and moral rights for the publications made accessible in the public portal are retained by the authors and/or other copyright owners and it is a condition of accessing publications that users recognise and abide by the legal requirements associated with these

- Users may download and print one copy of any publication from the public portal for the purpose of private study or research.

- You may not further distribute the material or use it for any profit-making activity or commercial gain

If the publication is distributed under the terms of Article $25 \mathrm{fa}$ of the Dutch Copyright Act, indicated by the "Taverne" license above, 


\section{Organ saving treatment after}

chemoradiotherapy for rectal cancer

- MRI as a selection tool - 
(C) copyright D.M.J. Lambregts, Maastricht 2013

Universitaire Pers Maastricht

ISBN 9789461592187

Printing: Datawyse / Universitaire Pers Maastricht

Omslagontwerp: Remko von Berg (remkoo.nl) 


\title{
Organ saving treatment after
}

\section{chemoradiotherapy for rectal cancer}

\section{- MRI as a selection tool -}

\author{
Proefschrift
}

Ter verkrijging van de graad van doctor aan de Universiteit Maastricht op gezag van de Rector Magnificus Prof. dr. L.L.G. Soete, volgens het besluit van het College van Decanen,

in het openbaar te verdedigen op woensdag 8 mei 2013 om 14.00 uur

door

Doenja Marina Johanna Lambregts

Geboren op 4 november 1983 te Breda

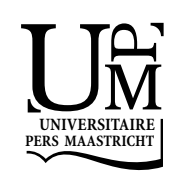




\section{Promotor}

Prof. dr. R.G.H. Beets-Tan

\section{Co-promotor}

Dr. G.L. Beets

\section{Beoordelingscommissie}

Prof. dr. J.M.A. van Engelshoven (voorzitter)

Prof. dr. K. Haustermans, UZ Leuven

Prof. dr. F.M. Mottaghy, RWTH Aachen / UM

Prof. dr. K. Nicolay, TU Eindhoven

Dr. H.J.T. Rutten, Catharina Ziekenhuis Eindhoven 


\section{Contents}

Chapter 1 Introduction and aim of the thesis .................................................

\section{PART I - TUMOUR RESPONSE}

Chapter 2 Diffusion-weighted MRI for selection of complete responders after chemoradiation for locally advanced rectal cancer: a multicenter study

Chapter 3 Rectal cancer: assessment of complete response to preoperative combined radiation therapy with chemotherapy: conventional MR volumetry versus diffusion-weighted MR imaging

Chapter 4 Tumour ADC measurements in rectal cancer: effect of ROI methods on $A D C$ values and interobserver variability

\section{PART II - LYMPH NODES}

Chapter 5 Value of ADC measurements for nodal staging after chemoradiation in locally advanced rectal cancer - a per lesion validation study

Chapter 6 Accuracy of gadofosveset-enhanced MRI for nodal staging and restaging in rectal cancer.

\section{PART III - FOLLOW-UP}

Chapter 7 Long-term follow-up features on rectal MRI during a wait-andsee approach after a clinical complete response in rectal cancer patients treated with chemoradiotherapy

Chapter 8 Value of MRI and diffusion-weighted MRI for the diagnosis of locally recurrent rectal cancer.

Chapter 9 Discussion and recommendations.

Summary / Samenvatting .....

Dankbetuigingen

Curriculum vitae

List of publications. 

INTRODUCTION AND AIM OF THE THESIS

\section{CHAPTER}




\section{Introduction}

Per year, approximately 3000 patients are diagnosed with rectal cancer in the Netherlands. ${ }^{1}$ For a long time the treatment for the whole patient group has been a blunt surgical resection of the rectum and tumour: a 'one size fits all' strategy. The main concern with this strategy was the relatively high local recurrence rates after rectal cancer surgery, reported to range from $10 \%$ to up to $40 \%{ }^{2}$ These local recurrences were mainly caused by incomplete resection of the tumour. With the introduction of standardised total mesorectal excision (TME) and the addition of preoperative radio- and/or chemotherapy the local recurrence rates dropped to below $10 \%{ }^{3,4}$ The aim of preoperative treatment is to induce tumour downsizing and downstaging in order to increase the number of complete (R0) resections. In patients with high risk, locally advanced tumours a long course of combined chemoradiation therapy (CRT) followed by TME is now considered the standard of treatment. Patients with low or intermediate risk tumours are treated either with TME only or TME preceded by a short course of radiation treatment. The required treatment for each individual patient is based on the tumour stage as determined by preoperative imaging. Worldwide, magnetic resonance imaging (MRI) is well adopted as a standard treatment stratification tool. Additionally, endorectal ultrasound (EUS) is used for the assessment of small, superficial tumours.

As a result of current chemoradiation schemes, the locally advanced tumours often show a phenomenal response. In up to $24 \%$ of patients no residual tumour cells are found in the resection specimen. ${ }^{5}$ These complete responders are known to have a better prognostic outcome than patients with residual tumour. ${ }^{5}$ The above findings have opened the debate whether it is still necessary in this selected well responding patient group to resect the whole rectum and expose patients to the associated morbidity and mortality. The first observational studies that have applied a smaller, local excision (transanal endoscopic microsurgery; ' $T E M$ ') in patients with a good response after chemoradiation (yT1-2N0) or a nonoperative 'wait-and-see' approach in patients with a complete tumour response (yTONO) showed very promising results. ${ }^{6-9}$ With such minimally invasive, organ saving treatments, morbidity and mortality can be significantly reduced without compromising outcome. Despite these potential benefits, clinicians remain reluctant to opt for organ saving treatment strategies. This is partly because level one evidence from randomised controlled trials supporting their efficacy is still lacking. Moreover, to safely perform organ saving treatments, an accurate patient selection is critical. If the tumour response to chemoradiotherapy would be overestimated and/or non-sterilised locoregional lymph nodes overlooked, patients would not receive a proper TME resection, thus exposing them to an unnecessary 
high risk for local and/or nodal recurrence. If the tumour response is underestimated, the good or complete responding patients will not be considered for local excision or wait-and-see, even though these might actually be good treatment alternatives with associated reduced morbidity and mortality rates.

To date, treatment response after completion of chemoradiotherapy is mainly assessed by clinical examination (digital rectal examination) and endoscopy + biopsies. Digital rectal examination is, however, known to underestimate the tumour response in the majority of patients. ${ }^{10}$ Endoscopic assessment after chemoradiation is not always accurate, especially if a fibrotic mass has replaced the former tumour bed. Biopsies are subject to sampling errors. ${ }^{11}$ The role of imaging for response evaluation after CRT is not yet consolidated. Until recently, response assessment with imaging was not so much of an issue because the imaging findings would not have altered the treatment strategy. Surgical resection was routinely performed, regardless of the response to CRT. Meanwhile, studies have shown that MRI can be beneficial to visualise tumour shrinkage and downstaging. In many centres these findings are now used to consider alteration of the surgical approach from a more extensive resection to a standard TME resection or - for the low tumours that show retraction from the anal sphincter - from an abdominoperineal to a sphincter preserving resection. ${ }^{12,13}$ Currently ongoing treatment developments have furthermore opened the discussion whether a restaging MRI could also be valuable to support the clinical selection of patients who may be considered for organ saving treatments in stead of a standard surgical resection: those patients with a significant treatment response or even a complete tumour regression. There are, however, two critical problems that need to be solved before imaging can truly prove its benefit in this regard.

First, there is the issue of nodal staging. It is known that in patients with a good (ypT1-2) and complete (ypT0) tumour response there is still a 15\% and 5\% chance, respectively, of a positive lymph node status after chemoradiotherapy. ${ }^{5}$ If remaining metastatic nodes are not identified, the risk for a local recurrence is significant regardless of the response of the primary tumour. ${ }^{14}$ Second, there is the problem of the interpretation of chemoradiation-induced fibrosis. Imaging cannot discriminate between fibrosis with or without (small) vital tumour remnants, while it is obviously critical to differentiate the two if patients are to be deferred from surgery and offered a 'wait-and-see' policy.

Standard anatomical/morphological (MR) imaging has only little value in solving these two issues. We know that the evaluation of nodal metastases with imaging, which is traditionally performed through size measurements, does not reach sensitivities and specificities higher than $55 \%$ and $78 \%$, respectively. Understaging of the nodal status occurs in up to $16 \%$ of patients using MRI and in up 
to $25 \%$ of patients using EUS. ${ }^{15,16,17}$ Furthermore, we know that MRI and EUS are insufficiently accurate to detect residual tumour after CRT, mainly due to above described difficulties in the interpretation of fibrosis. It is well known that in $50 \%$ of the cases in which the tumour bed has become fibrotic as a result of the CRT, this fibrosis may still contain small tumour remnants which are difficult to discern with imaging. As a result, limited sensitivities, ranging between 33 and 63\%, have been reported for MRI and EUS in detecting residual disease after CRT. ${ }^{11,18-20}$

Should organ saving treatments continue to set the pace, a precise selection of the eligible patients is a requirement. Imaging has the potential to complement clinical assessment tools, but only if it also proves beneficial in evaluating lymph nodes and identifying small residual disease. Since the morphological information obtained from conventional imaging methods is not sufficient, there is a need to investigate additional imaging techniques.

New functional imaging techniques in magnetic resonance imaging, such as diffusion-weighted MRI (DWI) and (dynamic) contrast-enhanced MRI, are on their way to become cornerstones in oncologic imaging. DWI is at the time of writing the technique most frequently investigated because of its potential value to discriminate between healthy and tumoural tissue types. DWI analyses the extracellular movement ('diffusion') of water protons, which differs dependent on a tissue's cellular density and microarchitecture. ${ }^{21}$ The diffusion of tissues can be quantitatively measured as the 'apparent diffusion coefficient' or 'ADC'. Since malignant tumours tend to have a higher cellular density than their surrounding tissues, they will exhibit different diffusion characteristics and ADC values. This 'functional' information can complement the morphological information from standard MRI and potentially help solve the problem of interpreting fibrosis. We hypothesise that - after CRT - the functional data from DWI combined with the morphological data from conventional MRI will improve the performance of imaging to detect small tumour remnants within the irradiated fibrotic tumour bed. The potential of DWI for assessment of lymph nodes has previously successfully been studied in head and neck and gynaecological tumours. ${ }^{22,23}$ However, the available evidence is not yet strong - even absent in rectal cancer nodes - and thorough lesion by lesion histological validation studies are lacking. Apart from DWI, the use of lymph node specific contrast agents (in specific Ultrasmall Superparamagnetic Particles of Iron Oxide or 'USPIO') has in prostate and rectal cancer studies proven valuable for nodal imaging. ${ }^{24,25}$ USPIO has, however, not been approved by the food and drug administration and is therefore not on the market. Our group has continued the search for potential contrast agents for nodal imaging and investigated an alternative MR contrast agent, gadofosveset trisodium, which is originally marketed for vascular MR imaging. Our feasibility study showed that 
gadofosveset can visualise tumoural nodes in rectal cancer patients and further research to investigate its diagnostic value for nodal staging is justified. ${ }^{26,27}$

\section{Aim of this thesis}

The aim of this thesis is to determine whether modern MR technology can improve the response evaluation of conventional MRI in patients with locally advanced rectal cancer preoperatively treated with chemoradiotherapy.

The two main study questions are:

1. What is the value of diffusion-weighted MRI for the assessment of the local tumour response after chemoradiation treatment in patients with locally advanced rectal cancer?

2. What is the value of diffusion-weighted MRI and MRI using a new lymph node contrast agent (gadofosveset trisodium) for the evaluation of lymph node status in patients with rectal cancer?

\section{Outline of this thesis}

\section{Part I - Tumour response}

Chapter 2 evaluates the diagnostic value of visual interpretation of DWI for tumour response evaluation after chemoradiation treatment and for the identification of patients who have undergone a complete tumour response.

In Chapter 3 the role of quantitative interpretation of DWI for tumour response evaluation is assessed by measuring tumour volumes on DWI, by analysing the tumour apparent diffusion coefficient $(A D C)$, and by comparing DWI to tumour volumetry performed on standard T2-weighted MRI.

Chapter 4 assesses the effect of different methods of measuring tumour ADCs on tumour ADC values and interobserver variability.

\section{Part II - Lymph nodes}

Chapter 5 evaluates the use of DWI for the discrimination between benign and malignant rectal cancer lymph nodes in the restaging of nodes after neoadjuvant chemoradiation treatment.

Chapter 6 describes the diagnostic value of lymph node contrast enhanced MRI using gadofosveset trisodium for the primary staging of rectal cancer lymph nodes and for the restaging of nodes after neoadjuvant chemoradiation treatment. 


\section{Part III - Follow-up}

In Chapter 7 the MR morphology of the rectal wall and its appearance on DWI are described during the short-term and long-term clinical follow-up of patients undergoing a wait-and-see policy.

Chapter 8 assesses the role of DWI for the diagnosis of locally recurrent rectal cancer during follow-up after primary treatment. 


\section{References}

1. Landelijke Werkgroep Gastro-Intestinale Tumoren. Landelijke Richtlijn Rectumcarcinoom (versie 2.1). www.oncoline.nl, 2008.

2. Quirke P, Durdey P, Dixon MF, Williams NS. Local recurrence of rectal adenocarcinoma due to inadequate surgical resection. Histopathological study of lateral tumour spread and surgical excision. Lancet 1986;2:996-9.

3. Heald RJ, Ryall RD. Recurrence and survival after total mesorectal excision for rectal cancer. Lancet 1986;1:1479-82.

4. Sauer R, Becker H, Hohenberger W, et al. Preoperative versus postoperative chemoradiotherapy for rectal cancer. N Engl J Med 2004;351:1731-40.

5. Maas M, Nelemans PJ, Valentini V, et al. Long-term outcome in patients with a pathological complete response after chemoradiation for rectal cancer: a pooled analysis of individual patient data. Lancet Oncol 2010;11:835-44.

6. Maas M, Beets-Tan RG, Lambregts DM, et al. Wait-and-See Policy for Clinical Complete Responders After Chemoradiation for Rectal Cancer. J Clin Oncol 2011;29:4633-40.

7. Habr-Gama A, Perez RO, Proscurshim I, et al. Patterns of failure and survival for nonoperative treatment of stage c0 distal rectal cancer following neoadjuvant chemoradiation therapy. J Gastrointest Surg 2006;10:1319-28; discussion 28-9.

8. Borschitz $T$, Wachtlin $D$, Mohler $M$, Schmidberger $H$, Junginger T. Neoadjuvant chemoradiation and local excision for T2-3 rectal cancer. Ann Surg Oncol 2008;15:712-20.

9. Lezoche $G$, Baldarelli M, Guerrieri $M$, et al. A prospective randomized study with a 5-year minimum follow-up evaluation of transanal endoscopic microsurgery versus laparoscopic total mesorectal excision after neoadjuvant therapy. Surg Endosc 2008;22:352-8.

10. Guillem JG, Chessin DB, Shia J, et al. Clinical examination following preoperative chemoradiation for rectal cancer is not a reliable surrogate end point. J Clin Oncol 2005;23:3475-9.

11. Maretto I, Pomerri F, Pucciarelli S, et al. The potential of restaging in the prediction of pathologic response after preoperative chemoradiotherapy for rectal cancer. Ann Surg Oncol 2007;14:455-61.

12. Park MJ, Kim SH, Lee SJ, Jang KM, Rhim H. Locally Advanced Rectal Cancer: Added Value of Diffusionweighted MR Imaging for Predicting Tumor Clearance of the Mesorectal Fascia after Neoadjuvant Chemotherapy and Radiation Therapy. Radiology 2011;260:771-80.

13. Vliegen RF, Beets GL, Lammering G, et al. Mesorectal fascia invasion after neoadjuvant chemotherapy and radiation therapy for locally advanced rectal cancer: accuracy of MR imaging for prediction. Radiology 2008;246:454-62.

14. Perez RO, Pereira DD, Proscurshim I, et al. Lymph node size in rectal cancer following neoadjuvant chemoradiation--can we rely on radiologic nodal staging after chemoradiation? Dis Colon Rectum 2009;52:1278-84.

15. Bipat S, Glas AS, Slors FJ, Zwinderman AH, Bossuyt PM, Stoker J. Rectal cancer: local staging and assessment of lymph node involvement with endoluminal US, CT, and MR imaging--a meta-analysis. Radiology 2004;232:773-83.

16. Lahaye MJ, Engelen SM, Nelemans PJ, et al. Imaging for predicting the risk factors--the circumferential resection margin and nodal disease--of local recurrence in rectal cancer: a metaanalysis. Semin Ultrasound CT MR 2005;26:259-68.

17. Guillem JG, Diaz-Gonzalez JA, Minsky BD, et al. cT3NO rectal cancer: potential overtreatment with preoperative chemoradiotherapy is warranted. J Clin Oncol 2008;26:368-73.

18. Vanagunas A, Lin DE, Stryker SJ. Accuracy of endoscopic ultrasound for restaging rectal cancer following neoadjuvant chemoradiation therapy. Am J Gastroenterol 2004;99:109-12.

19. Kim SH, Lee JM, Hong SH, et al. Locally advanced rectal cancer: added value of diffusion-weighted $\mathrm{MR}$ imaging in the evaluation of tumor response to neoadjuvant chemo- and radiation therapy. Radiology 2009;253:116-25. 
20. Suppiah A, Hunter IA, Cowley J, et al. Magnetic resonance imaging accuracy in assessing tumour down-staging following chemoradiation in rectal cancer. Colorectal Dis 2009;11:249-53.

21. Bammer R. Basic principles of diffusion-weighted imaging. Eur J Radiol 2003;45:169-84.

22. Vandecaveye V, De Keyzer F, Vander Poorten V, et al. Head and neck squamous cell carcinoma: value of diffusion-weighted MR imaging for nodal staging. Radiology 2009;251:134-46.

23. Kim JK, Kim KA, Park BW, Kim N, Cho KS. Feasibility of diffusion-weighted imaging in the differentiation of metastatic from nonmetastatic lymph nodes: early experience. J Magn Reson Imaging 2008;28:714-9.

24. Will O, Purkayastha S, Chan C, et al. Diagnostic precision of nanoparticle-enhanced MRI for lymphnode metastases: a meta-analysis. Lancet Oncol 2006;7:52-60.

25. Koh DM, Brown G, Collins DJ. Nanoparticles in rectal cancer imaging. Cancer Biomark 2009;5:89-98.

26. Lahaye MJ, Beets GL, Engelen SME, et al. Gadofosveset Trisodium (Vasovist ${ }^{\circledR}$ ) enhanced MR lymph node detection: initial observations. The Open Magnetic Resonance Journal 2009;2:1-5.

27. Herborn CU, Lauenstein TC, Vogt FM, Lauffer RB, Debatin JF, Ruehm SG. Interstitial MR lymphography with MS-325: characterization of normal and tumor-invaded lymph nodes in a rabbit model. AJR Am J Roentgenol 2002;179:1567-72. 
TUMOUR RESPONSE 

DIFFUSION-WEIGHTED MRI FOR SELECTION OF COMPLETE RESPONDERS AFTER CHEMORADIATION FOR LOCALLY ADVANCED RECTAL CANCER: A MULTICENTER STUDY

\section{CHAPTER}

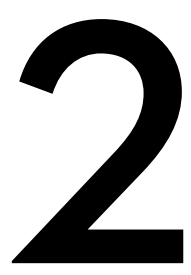

DMJ Lambregts $\checkmark$ Vandecaveye

B Barbaro FCH Bakers M Lambrecht MMaas

K Haustermans $\checkmark$ Valentini GL Beets RGH Beets-Tan

Published in: Annals of Surgical Oncology 2011; 18(8):2224-2231 


\section{Abstract}

\section{Aim}

In $10-24 \%$ of patients with rectal cancer who are treated with neoadjuvant chemoradiation, no residual tumour is found after surgery (ypT0). When accurately selected, these complete responders might be considered for less invasive treatments instead of standard surgery. So far, no imaging method has proven reliable. This study was designed to assess the accuracy of diffusion-weighted MRI (DWI) in addition to standard rectal MRI for selection of complete responders after chemoradiation.

\section{Materials \& Methods}

A total of 120 patients with locally advanced rectal cancer from three university hospitals underwent chemoradiation followed by a restaging MRI (1.5T), consisting of standard T2W-MRI and DWI (b0-1000). Three independent readers first scored the standard MRI only for the likelihood of a complete response using a 5-point confidence score, after which the DWI images were added and the scoring was repeated. Histology (урT0 vs. ypT1-4) was the standard reference. Diagnostic performance for selection of complete responders and interobserver agreement were compared for the two readings.

\section{Results}

Twenty-five of 120 patients had a complete response (урT0). Areas under the ROCcurve for the three readers improved from $0.76,0.68$, and 0.58 , using only standard $\mathrm{MRI}$, to $0.80,0.80$, and 0.78 after addition of DWI $(P=0.39,0.02$, and 0.002$)$. Sensitivity for selection of complete responders ranged from $0-40 \%$ on standard MRI versus 52-64\% after addition of DWI. Specificity was equally high (89-98\%) for both reading sessions. Interobserver agreement improved from $\mathrm{k}$ 0.20-0.32 on standard MRI to $0.51-0.55$ after addition of DWI.

\section{Conclusions}

Addition of DWI to standard rectal MRI improves the selection of complete responders after chemoradiation. 


\section{Introduction}

The introduction of preoperative, rather than postoperative adjuvant chemoradiation therapy (CRT) has led to a reduction in local recurrence rates and has become standard of care for patients with locally advanced rectal cancer. ${ }^{1}$ In $10-24 \%$ of patients, no residual tumour is found at histology after surgery. ${ }^{2}$ These complete responders are known to have a very good prognosis, in terms of overall and disease free survival. ${ }^{2}$ A complete response also raises the hotly debated question whether surgery is still necessary in these patients, especially since a total mesorectal excision (TME) may hold associated morbidity and even mortality and carries the potential risk of a permanent colostomy. Recently, a more conservative treatment is advocated in patients that show a good or even complete response to neoadjuvant treatment. In 2006, Habr-Gama et al presented the long-term results of a prospective trial investigating a 'wait-and-see' policy in a carefully selected group of patients with clinical and radiological evidence of a complete response after neoadjuvant CRT. Results at 5 year follow up were favourable for the nonsurgical group, with an overall and disease free survival of $93 \%$ and $85 \%$, respectively. ${ }^{3}$ In order to safely omit surgery it is essential to accurately select the right candidates, i.e. the true complete responders. This selection is currently mainly being done with digital examination, endoscopy and biopsy, but these methods are not infallible. The role of imaging for restaging after CRT has been the subject of several studies and all suggest that neither MRI nor endorectal ultrasound or ${ }^{18}$ F-Fluorodeoxyglucose-Positron Emission Tomography (FDG-PET) are sufficiently accurate for identifying the true complete responders with positive predictive values ranging between $17 \%$ and $50 \% .^{4-9}$ The use of these modalities for selection of patients would consequently put them at risk for undertreatment.

Diffusion-weighted MRI (DWI) is a functional MR imaging technique that uses differences in the extracellular movement of water protons to discriminate between tissues of varying cellularity. In tissues with a normal cellularity water protons can diffuse relatively freely, which results in a loss of signal on DWI. Conversely, in tissues with an increased cellularity (tumour) the diffusion of water is restricted, resulting in remaining high signal on DWI. In many reports, DWI has shown promise for identification of malignant tumours and recent studies on rectal cancer have indicated that DWI may also be useful for response evaluation after chemoradiation treatment. ${ }^{8,10-15}$ In 2009, Kim et al. showed in a study of 40 patients, that DWI in addition to standard MRI significantly improved the performance of radiologists to select complete responders when compared to standard MRI only. ${ }^{8}$ 
The purpose of our study was to evaluate the accuracy of DWI in addition to a standard restaging MRI for selection of complete responders after chemoradiation for locally advanced rectal cancer in a larger and multicenter study setting.

\section{Materials \& Methods}

\section{Patients}

This study retrospectively evaluated 120 consecutive patients who were treated for locally advanced rectal cancer in three university hospitals between 2005 and 2009. Due to the retrospective nature of the study, informed consent was not required. Ninety-three patients were men and 27 were women. Median age was 67 (range 22-89) years. Inclusion criteria consisted of (a) biopsy proven rectal cancer, (b) locally advanced disease as determined on primary staging MRI (T3-4 tumour, tumour involvement of the mesorectal fascia, and/or positive nodal status), (c) preoperative treatment consisting of a long course of neoadjuvant chemoradiation treatment, and (d) availability of post-treatment MR imaging including DWI. Exclusion criteria consisted of (a) non-resectable disease and (b) insufficient MR image quality (e.g. due to metal or motion artefacts). All patients underwent a long course of preoperative chemoradiation, consisting of capecitabine and/or oxaliplatin, combined with 50.4-55 Gy of radiation. After a 5-10 weeks time interval, all patients underwent a second, restaging MRI, including DWI, for response evaluation. Patients were then referred for further treatment.

\section{MR imaging}

In each participating centre, imaging was performed at 1.5T using a phased array body coil. The MR protocol consisted of standard T2-weighted fast spin echo sequences (as described in literature) in 3 orthogonal directions; sagittal, axial, and coronal with an in plane resolution ranging from $0.42-2.56 \mathrm{~mm}^{2}$ and a slice thickness of 4-5 mm. ${ }^{16}$ An additional diffusion-weighted echo planar imaging sequence was acquired with b0 as the lowest and b1000 s/mm as the highest b-factor, an in plane resolution of 7.8-9.6 $\mathrm{mm}^{2}$ and a slice thickness of 4-5 $\mathrm{mm}$, as described in previous reports from the participating centers. ${ }^{17-19}$

\section{Image evaluation}

All images were independently analysed by three readers, who were blinded to all clinical information, other imaging results, and histopathology. Reader 1 (RGHB) was a gastrointestinal (GI) radiologist who was highly specialised - with 13 years of 
experience - in reading pelvic MRI. Reader 2 (FCHB) was a GI radiologist with 3 years of experience in reading pelvic MRI. Reader 3 (VV) was a GI radiologist with 2 years pelvic MRI expertise and 5 years of experience in reading DWI images in head and neck, abdominal cancer and lymphoma. The three readers first evaluated the standard postchemoradiation (restaging) MR images and scored the likelihood of a complete response of the primary tumour using a confidence level score ( 0 = definitely residual tumour, $1=$ probably residual tumour, $2=$ possibly residual tumour/possibly complete response, 3 = probably complete response, $4=$ definitely complete response). The pre-CRT images were at the readers' disposal in order to identify the primary tumour, which is just like the evaluation process performed in daily clinical practice. Subsequently, the confidence level based scoring of the restaging MRI was repeated after addition of the b1000 DWI images.

\section{Imaging criteria}

On standard MRI, a normalised rectal wall without any detectable wall thickening was considered a definite criterion for a complete response (Figure 1). A solid residual mass with intermediate signal intensity on T2-weighted MRI was considered a definite criterion for residual tumour (Figure 2). Hypo-intense signal intensity changes indicated fibrosis, in which case undetermined scores were assigned (figure 3). ${ }^{18}$ On the diffusion images, residual high signal intensity on the location of the primary tumour was considered a criterion for residual tumour, while the absence of increased signal on DWI was indicative of a complete response (Figure 3). The readers assigned a confidence level 2 score ('equivocal score') when they were not able to differentiate between a complete response or residual tumour.

\section{Reference standard}

Histopathologic evaluation of the surgical resection specimen, according to the TNM staging system, served as the reference standard. The tumour regression grade (TRG) was evaluated according to the method of Mandard. ${ }^{20}$ The response of the primary tumour to chemoradiation was graded as follows: 'pathologic complete response' (= ypT0 / TRG 1, no residual tumour cells) or 'residual tumour' (= ypT1-4 / TRG 2-5, varying from limited tumour cells to a solid residual tumour mass). Eight patients did not undergo surgery, due to strong clinical evidence of a complete response (repeated negative sigmoidoscopy and biopsies after CRT). For these 8 patients, a local and distant recurrence free follow-up period of $>24$ months was considered a surrogate endpoint for a complete response. 

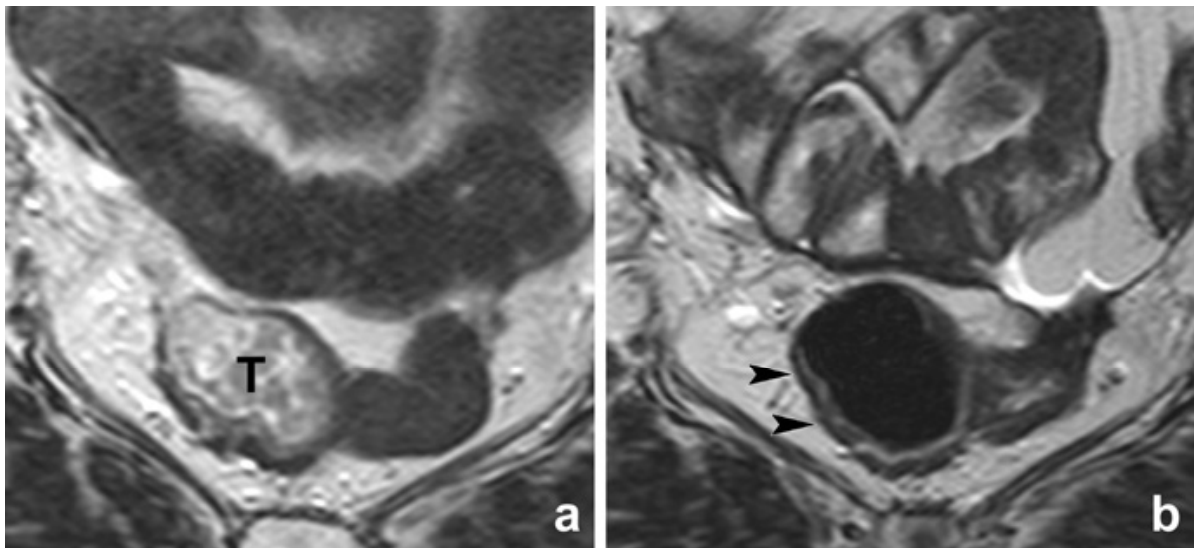

Figure 1. Standard T2-weighted images of a female patient with a tumour (T) in the mid-rectum, before (a) and after (b) preoperative chemoradiation therapy. After chemoradiation, the tumour has completely disappeared and a normalised rectal wall can be visualised (arrowheads). This feature was considered strongly predictive for a complete tumour response.
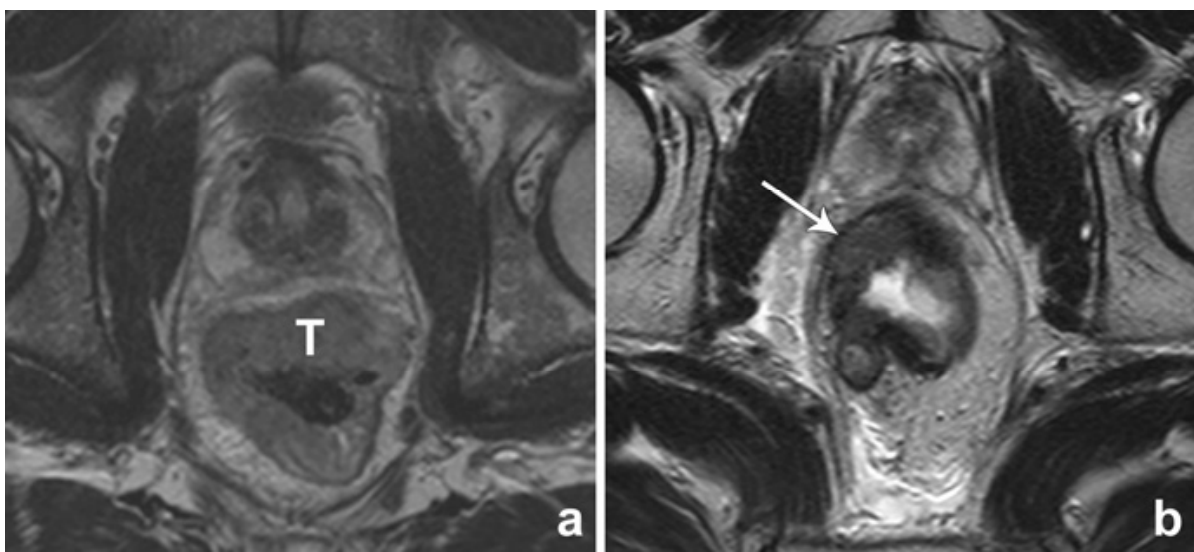

Figure 2. Standard T2-weighted images of a male patient with a tumour ( $T$ ) in the rectum, before (a) and after (b) preoperative chemoradiation therapy. After chemoradiation, a solid residual tumour mass is still visualised (arrow). This feature was considered strongly predictive for the presence of residual tumour.

\section{Statistical analyses}

Statistical analyses were performed using the Statistical Package for the Social Sciences (SPSS, version 16.0, Inc., Chicago, IL). Receiver operator characteristics (ROC) curve analyses were performed to evaluate the diagnostic performance of (a) standard MRI only and (b) standard MRI + DWI, for identification of a complete response. Corresponding areas under the ROC-curve (AUC), sensitivities, specificities, positive predictive values (PPV) and negative predictive values (NPV) 
with $95 \%$ confidence intervals $(\mathrm{Cl})$ were calculated. For these analyses it had been decided at the start of the study to dichotomise the confidence level scores between 2 (possibly residual tumour/possibly complete response) and 3 (probably complete response). Differences in diagnostic performance between standard MRI only and the combination of standard MRI + DWI were analysed by comparing the ROC-curves according to the method described by DeLong et al. ${ }^{21}$ P-values $<0.05$ were considered statistically significant. Weighted kappa values with quadratic kappa weighting (0.00-0.20 poor, 0.21-0.40 fair, 0.41-0.60 moderate, 0.61-0.80 good and $0.81-1.00$ excellent agreement) were calculated to evaluate interobserver variability. $^{22}$
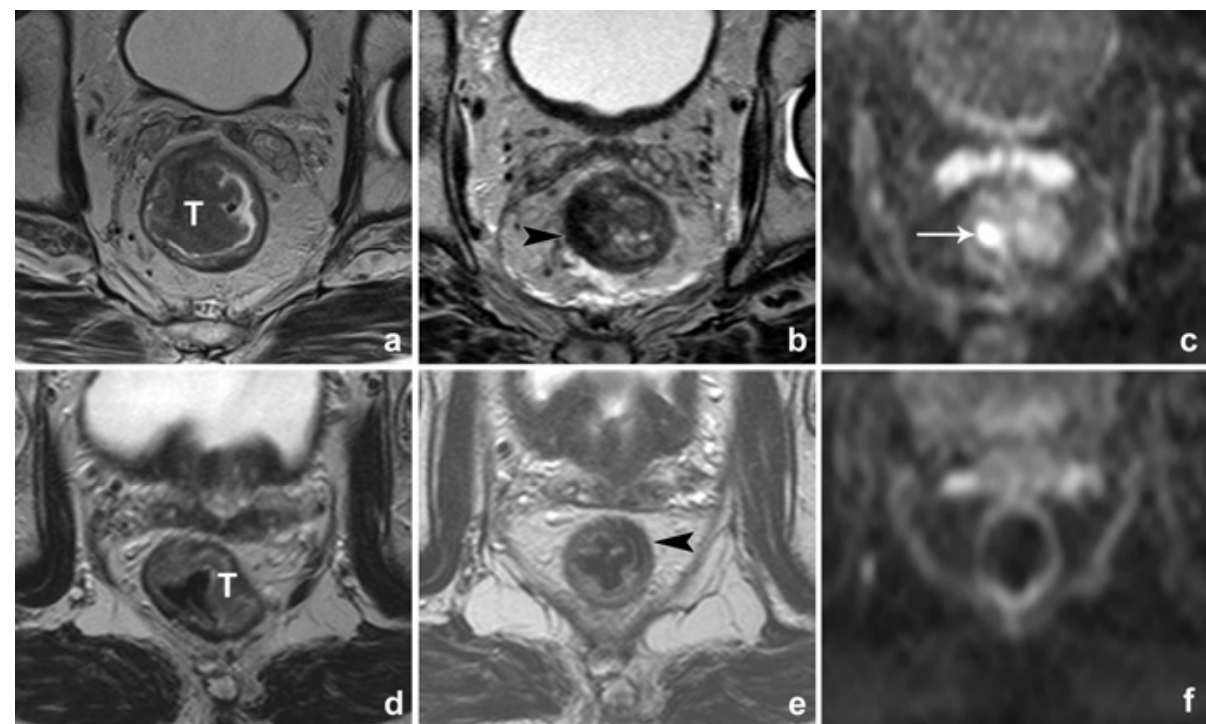

Figure 3. Standard T2-weighted images of two patients with a tumour ( $T$ ) in the rectum before (a,d) and after chemoradiation treatment (b,e). In both cases the tumour bed has become fibrotic after chemoradiation (arrowheads), which makes it difficult to discriminate between residual tumour and a complete response. In the upper patient, there is still a clear high signal intensity area on DWI (arrow in c), which was confirmed to be a ypT2 residual tumour at histology. In the lower patient, no high signal is shown on DWI (f) and a complete tumour response (ypT0) was confirmed at histology.

\section{Results}

\section{Patient and treatment characteristics}

79 patients underwent a low anterior resection, 25 an abdominoperineal resection, 4 more extended surgery and 4 a local excision (transanal endoscopic microsurgery). At histology 17 patients had a ypT0, 11 ypT1, 25 ypT2, 55 yрT3 and 4 a ypT4 status. 
Ten patients had mucinous type adenocarcinoma. The median time interval between the restaging MRI and surgery was 15 days (range 0-61). The eight patients who did not undergo surgery, had a median local and distant recurrence free followup of 42.5 months (range 26-73); these patients were therefore considered complete responders. All together 25 patients had a complete response and 95 had residual tumour. Of the patients with residual tumour, 63 had a yN0, 22 yN1 and 10 a yN2 status. Of the patients with a complete tumour response, 23 had a yN0, 1 yN1 and 1 a yN2 status. There were no significant differences in patient characteristics, gender or age distribution between the separate centers.
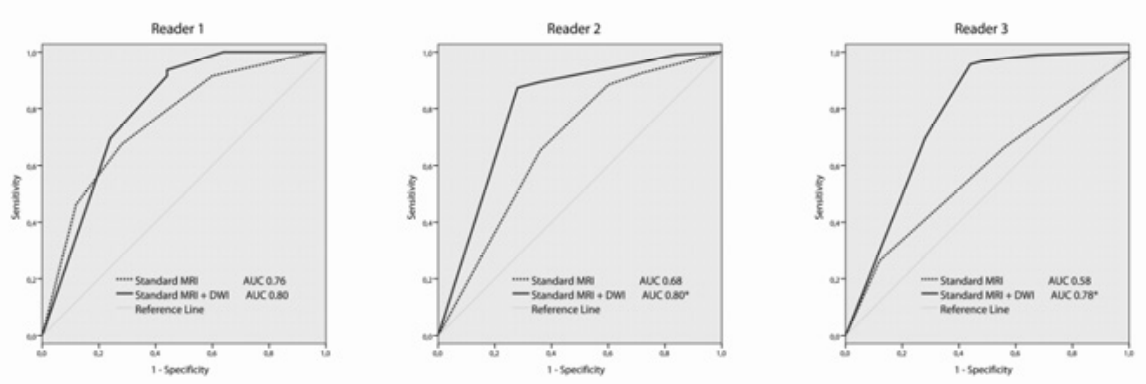

Figure 4. Receiver operator characteristics curves and areas under the curve (AUC) of the three readers for identification of a complete tumour response after CRT using only standard MRI and standard MRI + DWI, respectively. Diagnostic performance improved significantly $(*)$ for reader $2(P=0.02)$ and reader 3 $(P=0.002)$. For reader 1 there was no significant improvement $(P=0.39)$.

\section{Diagnostic performance for selection of complete responders}

ROC-curves for the selection of complete responders are displayed in figure 4 . Corresponding accuracy figures and AUCs with 95\% confidence intervals are provided in Table 1. For the highly expert reader 1, AUC improved from 0.76 for standard MRI to 0.80 for standard MRI + DWI $(P=0.39)$. For the less experienced reader 2, AUC improved from 0.68 on standard $M R I$ to 0.80 after addition of DWI $(P=0.02)$. For reader $3, A U C$ improved from 0.58 on standard MRI to 0.78 after addition of DWI $(P=0.002)$. 
Table 1. Diagnostic performance for the prediction of a complete response (ypT0)

\begin{tabular}{|l|c|c|c|c|c|c|c|}
\hline & \multicolumn{5}{|c|}{ Standard MRI only } & \multicolumn{3}{c|}{ Standard MRI + DWI } \\
\hline & R1 & R2 & R3 & R1 & R2 & R3 \\
\hline Sensitivity & $40(10 / 25)$ & $28(7 / 25)$ & $0(0 / 25)$ & $56(14 / 25)$ & $64(16 / 25)$ & $52(13 / 25)$ \\
\hline $95 \% \mathrm{Cl}$ & $26-53$ & $16-40$ & $0-0$ & $41-67$ & $48-77$ & $39-60$ \\
\hline Specificity & $92(87 / 95)$ & $93(88 / 95)$ & $98(93 / 95)$ & $94(89 / 95)$ & $89(85 / 95)$ & $97(92 / 95)$ \\
\hline $95 \% \mathrm{Cl}$ & $88-95$ & $89-96$ & $98-99$ & $90-97$ & $85-93$ & $93-99$ \\
\hline PPV & $56(10 / 18)$ & $50(7 / 14)$ & $0(0 / 2)$ & $70(14 / 20)$ & $62(16 / 26)$ & $81(13 / 16)$ \\
\hline $95 \% \mathrm{Cl}$ & $36-73$ & $28-71$ & $0-0$ & $52-84$ & $46-74$ & $60-93$ \\
\hline NPV & $85(87 / 102)$ & $83(88 / 106)$ & $79(93 / 118)$ & $89(89 / 100)$ & $90(85 / 94)$ & $88(92 / 104)$ \\
\hline $95 \% \mathrm{Cl}$ & $82-88$ & $80-86$ & $79-80$ & $85-92$ & $86-94$ & $85-90$ \\
\hline AUC & 0.76 & 0.68 & 0.58 & 0.80 & $0.80 *$ & $0.78 *$ \\
\hline $95 \% \mathrm{Cl}$ & $0.65-0.86$ & $0.56-0.80$ & $0.47-0.69$ & $0.69-0.91$ & $0.70-0.91$ & $0.67-0.90$ \\
\hline
\end{tabular}

$\mathrm{R} 1$; reader 1, GI radiologist with 13 years experience in pelvic MRI. R2; reader 2, GI radiologist with 3 years experience in pelvic MRI. R3; reader $3, \mathrm{GI}$ radiologist with 2 years experience in pelvic MRI and 5 years experience in reading DWI.

PPV = positive predictive value, NPV = negative predictive value, AUC = area under the ROC curve, 95\% $\mathrm{Cl}=95 \%$ confidence interval. Numbers are percentages, absolute numbers are given in parentheses.

* indicates a significant difference in AUC compared with standard MRI

\section{Number of equivocal (confidence level 2) scores}

When using only standard MRI without DWI, reader 1,2 and 3 assigned a confidence level score of 2 (possibly residual tumour/possibly complete response) to 31,7 and 41 patients, respectively. After addition of DWI, the number of equivocal scores decreased to 2,4 and 2 , for the three readers, respectively. This resulted in a reduced number of false negatives for prediction of a complete tumour response, ranging from 9-12 for the three readers on standard MRI + DWI as compared to $15-25$ on standard MRI only. The number of false positives remained unchanged and ranged from 2-8 on standard MRI and from 3-10 after addition of DWI.

\section{Interobserver agreement}

Kappa values for the interobserver agreement between the three readers are displayed in Table 2. Interobserver agreement improved from fair agreement 
( $\mathrm{k} 0.20-0.32$ ) on standard MRI to moderate agreement ( $\mathrm{k} 0.51-0.55)$ after addition of DWI.

Table 2. Interobserver agreement between the three readers.

\begin{tabular}{|c|c|c|}
\hline Observers & Standard MRI only (к) & Standard MRI + DWI (к) \\
\hline R1 \& R2 & 0.32 & 0.55 \\
\hline R1 \& R3 & 0.31 & 0.52 \\
\hline R2 \& R3 & 0.20 & 0.51 \\
\hline
\end{tabular}

$\mathrm{R} 1$; reader 1, GI radiologist with 13 years experience in pelvic MRI. R2; reader 2, GI radiologist with 3 years experience in pelvic MRI. R3; readers $3, \mathrm{GI}$ radiologist with 2 years experience in pelvic MRI and 5 years experience in reading DWI. Kappa values are weighted kappa's with quadratic kappa weighting.

\section{Discussion}

The findings of this study indicate that the diagnostic performance for predicting a pathologic complete tumour response after chemoradiation improved for the combination of standard MRI + DWI (AUC 0.78-0.80) as compared to standard MRI only (AUC 0.58-0.76). With the addition of DWI, sensitivity for identification of a complete response improved by $16-52 \%$ for the three readers. Moreover, it resulted in a substantial reduction in the number of equivocal scores and an improved interobserver agreement.

Of interest is the improved sensitivity for the combination of MRI + DWI; i.e. it resulted in less overestimation of tumour in patients with a complete tumour response. This is mainly because on the restaging MRI without DWI, many interpretation difficulties were observed when the primary tumour bed had become fibrotic as a result of the radiation treatment. In these cases, it is difficult to differentiate small areas of residual tumour from mere fibrosis and readers tend to overestimate the presence of tumour (Figure 3). ${ }^{23-26}$ Apparently, this is where the functional information from DWI proves beneficial. Areas of fibrosis typically have a low cellular density, which results in low signal intensity on high b-value (b1000) diffusion images. ${ }^{27}$ Conversely, residual tumour areas have a relatively high cellular density and show high signal on DWI, which stands out within the low signal of the surrounding tissue/fibrosis. This is the reason why small areas of residual tumour are better depicted on DWI. ${ }^{8,27}$ Nevertheless, interpretation errors were still observed with DWI resulting in a suboptimal sensitivity of $52-64 \%$. When the signal of the normal rectal wall is not fully suppressed on DWI, which often occurs when the rectal wall is collapsed, high signal at the location of the initial tumour area may 
erroneously be interpreted as residual tumour, resulting in overstaging errors. In addition, some imaging artifacts may occur on DWI, particularly around air-tissue interfaces. It is relevant to recognise these shortcomings of DWI and initiate teaching courses in which radiologists will be trained in the interpretation of DWI and will become familiar with its pitfalls. Specificity for MRI and DWI is above $90 \%$, indicating that the residual tumours are accurately detected and the risk for undertreatment will be less than $10 \%$. Although DWI allows detection of even small (2-5 $\mathrm{mm}$ ) tumour volumes, the challenge will remain the detection of microscopically small clusters of residual tumour cells, that are difficult to detect even at histology - and are currently beyond the detection level of any available imaging modality, including DWI.

The addition of DWI improved the performance of all readers, albeit that this benefit was not significant for reader 1 . His extensive experience of 13 years in interpreting rectal cancer MRI may explain why reader 1 was already more accurate with the use of only standard MRI (AUC 0.76). This exceptionally high level of expertise does not reflect common daily practice. Our study, however, clearly shows that for radiologists in general centers with expertise levels like the other 2 readers, DWI can really be of value. Furthermore, all readers showed a significant reduction in equivocal (confidence level 2) scores after addition of DWI, indicating that it raised their confidence in the discrimination between complete responders and residual tumour. This also explains the better interobserver agreement between the readers after addition of DWI.

So far, the largest body of evidence for response evaluation exists for ${ }^{18}$ FDGPET. Changes in FDG uptake, in particular early ( \pm 2 weeks) after onset of treatment, have proven useful for prediction of response. ${ }^{4,28,29}$ PET is, however, less reliable in identifying the complete tumour responders after completion of chemoradiation: up to $55 \%$ of the residual tumours are overlooked and patients are erroneously interpreted as complete responders. 5, 6, 28 In a recent study by Janssen et al, only 1 out of 6 complete responders as identified on FDG-PET corresponded with a true complete response at histology. ${ }^{4}$ When using FDG-PET for treatment planning, the main risk would thus be an undertreatment of these patients. In our DWI-MRI study, the presence of residual tumour was underestimated in only less than $10 \%$ of the cases, indicating that - compared to PET - there is a considerably smaller risk for undertreatment.

To the best of our knowledge this is the largest and only multicenter study investigating the value of DWI for identifying complete tumour responders after CRT for rectal cancer. It confirms previous findings of a smaller single center study by Kim et al. $^{8}$ Previous studies have also shown promise for quantitative DWI measurements of the 'apparent diffusion coefficient' (ADC) (performed pre, during 
and/or after chemoradiation treatment) to predict the degree of response to therapy. ${ }^{8,10-15,17,30,31}$ In our study we only focussed on qualitative, visual evaluation of DWI and did not quantitatively measure ADC. This is a more convenient approach, since a visual analysis is more practical and less time consuming for a busy radiology practice. Furthermore, ADC values are dependant on technical variations among DWI sequences generated by different MR equipment. ADC data from multiple centres may therefore be less suitable for pooled analysis. Visual evaluation of DWI images is less subjected to technical variations and pooling of these data was feasible because all three participating centres acquired a DWI sequence with equal (b1000) diffusion weighting. Nevertheless, we acknowledge that small variations between the participating centers may have introduced some bias.

In the current study we only focused on response assessment of the primary tumour and not the lymph nodes. The prevalence of a positive lymph node status in case of a complete response of the primary tumour after CRT is very low and was only $2 / 25(8 \%)$ in the present study. Nevertheless, in order to safely offer patients a 'wait-and-see' policy after CRT, we have to ensure that both the primary tumour and all metastatic nodes have undergone a complete regression (ypTONO). Although standard MRI is known to be inaccurate for the primary staging of rectal cancer nodes $^{32,33}$, evidence arises that after chemoradiation, MRI performs considerably better. High NPVs ranging between $81-100 \%$ have been reported, suggesting that the ypNO patients can already be accurately selected and the addition of functional techniques such as DWI may not even be necessary. ${ }^{7,19,23,34-36}$ Furthermore, the only study focussing specific on DWI for staging of rectal cancer nodes after CRT already showed good results for standard MRI only (NPV 94-95\%) and reported no clear benefit after addition of DWI (NPV 92-93\%). The main role of DWI for lymph node evaluation was that it improved the number of detected nodes (both benign and malignant), because nodes were more easily detected on DWI due to their high signal intensity compared to the suppressed background signal of surrounding tissues. $^{19}$

\section{Clinical impact}

A 'wait-and-see' approach ${ }^{3}$ or local excision ${ }^{37}$ for patients with a good response after chemoradiation is at present still debatable. Initiating and performing large patient studies to prove their efficiency is difficult, partly because clinicians are not convinced that safe selection of the right patients can be done. Therefore, one of the most important cornerstones to make implementation of such minimally invasive treatments possible is a precise selection of the eligible patients. Our aim 
was to assess whether MR imaging can be beneficial in this regard. Because of its reported promise in cancer imaging, we particularly looked at the potential of diffusion-weighted MRI. Moreover, DWI is a non-invasive technique that does not require the use of ionising radiation or contrast agents and can easily be added to any standard MRI protocol. Our results suggest that, by combining morphological with functional imaging information, MRI + DWI can significantly improve sensitivity for selection of complete responders. Furthermore, specificity is $>90 \%$, which indicates that the risk for underestimation of residual tumour can be brought to less than $10 \%$. As an adjunct to clinical tools (digital examination, endoscopy and biopsy), the combined use of MRI + DWI thus seems promising to enable a more precise selection of patients eligible to undergo minimally invasive treatments. The current results are obviously still premature for clinical decision making, but its promise warrants further large and prospective patient studies.

In conclusion, this study shows that the addition of diffusion-weighted imaging to a standard, restaging MRI improves the performance and confidence of radiologists in selecting the patients with a pathological complete tumour response after chemoradiation for locally advanced rectal cancer. The combination of MRI + DWI could therefore be of additional value for the clinical assessment of these patients.

\section{Acknowledgements}

The authors would like to thank Frederik de Keyzer for his assistance in the statistical data analyses. 


\section{References}

1. Sauer R, Becker H, Hohenberger W, et al; German Rectal Cancer Study Group. Preoperative versus postoperative chemoradiotherapy for rectal cancer. N Engl J Med 2004; 351:1731-1740.

2. Maas $\mathrm{M}$, Nelemans $\mathrm{P}$, Valentini $\mathrm{V}$, et al. Long-term outcome in patients with a pathological complete response after chemoradiation for rectal cancer: a pooled analysis of individual patient data. Lancet Oncol 2010; 11:835-844

3. Habr-Gama A, Perez RO, Proscurshim I, Campos FG, Nadalin W, Kiss D, Gama-Rodrigues J. Patterns of failure and survival for nonoperative treatment of stage $\mathrm{c} 0$ distal rectal cancer following neoadjuvant chemoradiation therapy. J Gastrointest Surg 2006; 10:1319-1328; discussion 28-29.

4. Janssen MH, Ollers MC, Riedl RG, et al. Accurate Prediction of Pathological Rectal Tumor Response after Two Weeks of Preoperative Radiochemotherapy Using (18)F-Fluorodeoxyglucose-Positron Emission Tomography-Computed Tomography Imaging. Int J Radiat Oncol Biol Phys 2010; 77:392399

5. Capirci C, Rubello D, Chierichetti F, et al. Restaging after neoadjuvant chemoradiotherapy for rectal adenocarcinoma: role of F18-FDG PET. Biomed Pharmacother 2004; 58:451-457.

6. Kristiansen C, Loft A, Berthelsen AK, Graff J, Lindebjerg J, Bisgaard C, Jakobsen A. PET/CT and histopathologic response to preoperative chemoradiation therapy in locally advanced rectal cancer. Dis Colon Rectum 2008; 51:21-25.

7. Suppiah A, Hunter IA, Cowley J, Garimella V, Cast J, Hartley JE, Monson JR. Magnetic resonance imaging accuracy in assessing tumour down-staging following chemoradiation in rectal cancer. Colorectal Dis 2009; 11:249-253.

8. Kim SH, Lee JM, Hong SH, Kim GH, Lee JY, Han JK, Choi BI. Locally advanced rectal cancer: added value of diffusion-weighted MR imaging in the evaluation of tumor response to neoadjuvant chemoand radiation therapy. Radiology 2009; 253:116-125.

9. Vanagunas A, Lin DE, Stryker SJ. Accuracy of endoscopic ultrasound for restaging rectal cancer following neoadjuvant chemoradiation therapy. Am J Gastroenterol 2004; 99:109-12.

10. Sun YS, Zhang XP, Tang L, Ji JF, Gu J, Cai Y, Zhang XY. Locally advanced rectal carcinoma treated with preoperative chemotherapy and radiation therapy: preliminary analysis of diffusion-weighted MR imaging for early detection of tumor histopathologic downstaging. Radiology 2010; 254:170-178.

11. DeVries AF, Kremser C, Hein PA, Griebel et al. Tumor microcirculation and diffusion predict therapy outcome for primary rectal carcinoma. Int J Radiat Oncol Biol Phys 2003; 56:958-965.

12. Hein PA, Kremser C, Judmaier W, et al. Diffusion-weighted magnetic resonance imaging for monitoring diffusion changes in rectal carcinoma during combined, preoperative chemoradiation: preliminary results of a prospective study. Eur J Radiol 2003; 45:214-222.

13. Dzik-Jurasz A, Domenig C, George M, Wolber J, Padhani A, Brown G, Doran S. Diffusion MRI for prediction of response of rectal cancer to chemoradiation. Lancet 2002; 360:307-308.

14. Patterson DM, Padhani AR, Collins DJ. Technology insight: water diffusion MRI--a potential new biomarker of response to cancer therapy. Nat Clin Pract Oncol 2008; 5:220-233.

15. Kremser $C$, Judmaier $W$, Hein $P$, Griebel J, Lukas $P$, de Vries A. Preliminary results on the influence of chemoradiation on apparent diffusion coefficients of primary rectal carcinoma measured by magnetic resonance imaging. Strahlenther Onkol 2003;179:641-649.

16. Brown G, Kirkham A, Williams GT, et al. High-resolution MRI of the anatomy important in total mesorectal excision of the rectum. AJR Am J Roentgenol 2004; 182:431-439.

17. Lambrecht M, Deroose $C$, Roels $S$, et al. The use of FDG-PET/CT and diffusion-weighted magnetic resonance imaging for response prediction before, during and after preoperative chemoradiotherapy for rectal cancer. Acta Oncol 2010; 49:956-963

18. Barbaro B, Vitale R, Leccisotti L, et al. Restaging locally advanced rectal cancer with MR imaging after chemoradiation therapy. Radiographics 2010; 30:699-716. 
19. Lambregts DMJ, Maas M, Riedl RG, et al. Value of ADC measurements for nodal staging after chemoradiation in locally advanced rectal cancer-a per lesion validation study. Eur Radiol 2010; 21:265-273

20. Mandard AM, Dalibard F, Mandard JC, et al. Pathologic assessment of tumor regression after preoperative chemoradiotherapy of esophageal carcinoma. Clinicopathologic correlations. Cancer 1994; 73:2680-2686.

21. DeLong ER, DeLong DM, Clarke-Pearson DL. Comparing the areas under two or more correlated receiver operating characteristic curves: a nonparametric approach. Biometrics 1988; 44:837-845.

22. Cohen J. Weighted kappa: nominal scale agreement with provision for scaled disagreement or partial credit. Psychol Bull 1968; 70:213-220.

23. Barbaro B, Fiorucci C, Tebala $C$, et al. Locally advanced rectal cancer: $M R$ imaging in prediction of response after preoperative chemotherapy and radiation therapy. Radiology 2009; 250:730-739.

24. Kuo LJ, Chern MC, Tsou MH, et al. Interpretation of magnetic resonance imaging for locally advanced rectal carcinoma after preoperative chemoradiation therapy. Dis Colon Rectum 2005; 48:23-28.

25. Dresen RC, Beets GL, Rutten HJ, et al. Locally advanced rectal cancer: MR imaging for restaging after neoadjuvant radiation therapy with concomitant chemotherapy. Part I. Are we able to predict tumor confined to the rectal wall? Radiology 2009; 252:71-80.

26. Jonas J, Bahr R. Neoadjuvant chemoradiation treatment impairs accuracy of MRI staging in rectal carcinoma. Gut 2006; 55:1214-1215.

27. Vandecaveye V, De Keyzer F, Nuyts S, et al. Detection of head and neck squamous cell carcinoma with diffusion weighted MRI after (chemo)radiotherapy: correlation between radiologic and histopathologic findings. Int J Radiat Oncol Biol Phys 2007; 67:960-971.

28. de Geus-Oei LF, Vriens D, van Laarhoven HW, van der Graaf WT, Oyen WJ. Monitoring and predicting response to therapy with 18 F-FDG PET in colorectal cancer: a systematic review. J Nucl Med 2009; 50(Suppl 1):43S-54S.

29. Cascini GL, Avallone A, Delrio $P$, et al. 18F-FDG PET is an early predictor of pathologic tumor response to preoperative radiochemotherapy in locally advanced rectal cancer. J Nucl Med 2006; 47:12411248

30. Roth $\mathrm{Y}$, Tichler $\mathrm{T}$, Kostenich $\mathrm{G}$, et al. High-b-value diffusion-weighted MR imaging for pretreatment prediction and early monitoring of tumor response to therapy in mice. Radiology 2004; 232:685-692.

31. Kim SH, Lee JY, Lee JM, Han JK, Choi BI. Apparent diffusion coefficient for evaluating tumour response to neoadjuvant chemoradiation therapy for locally advanced rectal cancer. Eur Radiol 2011; 21:987-95

32. Bipat S, Glas AS, Slors FJ, Zwinderman AH, Bossuyt PM, Stoker J. Rectal cancer: local staging and assessment of lymph node involvement with endoluminal US, CT, and MR imaging--a meta-analysis. Radiology 2004; 232:773-783.

33. Lahaye MJ, Engelen SM, Nelemans PJ, Beets GL, van de Velde CJ, van Engelshoven JM, Beets-Tan RG. Imaging for predicting the risk factors--the circumferential resection margin and nodal disease--of local recurrence in rectal cancer: a meta-analysis. Semin Ultrasound CT MR 2005; 26:259-268.

34. Lahaye MJ, Beets GL, Engelen SM, et al. Locally advanced rectal cancer: MR imaging for restaging after neoadjuvant radiation therapy with concomitant chemotherapy. Part II. What are the criteria to predict involved lymph nodes? Radiology 2009; 252:81-91.

35. Maretto I, Pomerri F, Pucciarelli S, et al. The potential of restaging in the prediction of pathologic response after preoperative chemoradiotherapy for rectal cancer. Ann Surg Oncol 2007; 14:455-461.

36. Denecke T, Rau B, Hoffmann KT, et al. Comparison of CT, MRI and FDG-PET in response prediction of patients with locally advanced rectal cancer after multimodal preoperative therapy: is there a benefit in using functional imaging? Eur Radiol 2005 ; 15:1658-1666.

37. Lezoche G, Baldarelli M, Guerrieri M, Paganini AM, De Sanctis A, Bartolacci S, Lezoche E. A prospective randomised study with a 5-year minimum follow-up evaluation of transanal endoscopic microsurgery versus laparoscopic total mesorectal excision after neoadjuvant therapy. Surg Endosc 2008; 22:352-358. 



\section{RECTAL CANCER: ASSESSMENT OF}

COMPLETE RESPONSE TO PREOPERATIVE COMBINED RADIATION THERAPY WITH CHEMOTHERAPY

-CONVENTIONAL MR VOLUMETRY VERSUS DIFFUSION-WEIGHTED MR IMAGING

\section{CHAPTER}

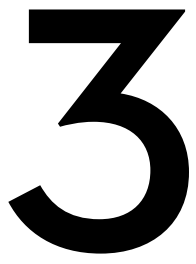

L Curvo-Semedo DMJ Lambregts M Maas T Thywissen RT Mehsen G Lammering GL Beets F Caseiro-Alves RGH Beets-Tan

Published in: Radiology 2011; 260(3):734-743 


\section{Abstract}

\section{Aim}

To determine the diagnostic performance of diffusion-weighted MRI (DWI) for the assessment of a complete tumour response (CR) after combined radiation therapy with chemotherapy (CRT) in patients with locally advanced rectal cancer (LARC) by means of volumetric signal intensity measurements and apparent diffusion coefficient $(A D C)$ measurements and to compare the performance of DW imaging with that of T2-weighted MR.

\section{Materials \& Methods}

A retrospective analysis of 50 patients with $L A R C$, for whom clinical and imaging data were retrieved from a previous imaging study approved by the local institutional ethical committee and for which all patients provided informed consent, was conducted. Patients underwent pre- and post-CRT standard T2weighted MR and DW MR. Two independent readers placed free-hand regions of interest (ROIs) in each tumour-containing section on both data sets to determine pre- and post-CRT tumour volumes and tumour volume reduction rates ( $\Delta$ volume). ROls were copied to an ADC map to calculate tumour ADCs. Histopathologic findings were the standard of reference. Receiver operating characteristic (ROC) curves were generated to compare performance of T2-weighted and DW MR volumetry and ADC. The intraclass correlation coefficient (ICC) was used to evaluate interobserver variability and the correlation between T2-weighted and DW MR volumetry.

\section{Results}

Areas under the ROC curve (AUCS) for identification of a CR that was based on preCRT volume, post-CRT volume, and $\Delta$ volume, respectively, were $0.57,0.70$, and 0.84 for T2-weighted MR versus $0.63,0.93$, and 0.92 for $D W$ MR volumetry $(P=0.15$, $0.02,0.42$ ). Pre- and post-CRT ADC and $\triangle A D C$ AUCs were 0.55, 0.54, and 0.51, respectively. Interobserver agreement was excellent for all pre-CRT measurements (ICC, 0.91-0.96) versus good (ICC, 0.61-0.79) for post-CRT measurements. ICC between T2-weighted and DW MR volumetry was excellent (0.97) for pre-CRT measurements versus fair $(0.25)$ for post-CRT measurements. 


\section{Conclusions}

Post-CRT DW MR volumetry provided high diagnostic performance in assessing CR and was significantly more accurate than T2-weighted MR volumetry. Post-CRT DW MR was equally as accurate as $\Delta$ volume measurements of both T2-weighted and DW MR. Pre-CRT volumetry and ADC were not reliable. 


\section{Introduction}

The treatment of locally advanced rectal cancer (LARC) has shifted in recent years from a primarily surgical approach with adjuvant radiation therapy toward preoperative combined radiation therapy with chemotherapy (CRT), which results in improved local control and reduced acute and late toxic effects. ${ }^{1}$ Moreover, the use of preoperative CRT induces downsizing and downstaging of the primary tumour, yielding a pathologic complete response (CR) (pCR) in up to $24 \%$ of patients. A pCR is known to be associated with a favorable oncologic outcome, in regard to both recurrence and survival. ${ }^{2}$ Habr-Gama et al reported on 99 patients with a clinical CR who were treated with observation alone ("wait-and-see"): Compared with patients who had residual tumour and were referred to surgery, the 5-year overall and disease-free survival rates were favorable for the observation group. ${ }^{3}$ Although still controversial, the trend in treatment is now toward a more conservative policy for patients identified as complete responders after CRT. ${ }^{4-6}$ Traditionally, a pCR is determined with histopathologic examination after surgery. However, if the determination of a CR before surgery would influence the subsequent treatment choice, an accurate clinical assessment of response becomes essential. Currently used methods, such as digital examination and endoscopy and/or biopsy are good but not infallible. The role of magnetic resonance (MR) imaging in the primary staging of rectal cancer is well established, and it is now part of the standard workup in many countries. However, its role in restaging after preoperative CRT is not yet clear, partly because to date restaging by using imaging has not influenced the treatment strategy. MR imaging, like other morphologic imaging techniques (endorectal ultrasonography and computed tomography) is hampered by interpretation difficulties in assessing the presence of residual tumour within areas of radiation-induced fibrosis. ${ }^{7-9}$ Studies are therefore focusing on the potential added benefit of functional and/or quantitative methods of MR image evaluation. One of these, MR volumetry, was reported to correlate well with downstaging of rectal cancer: A tumour volume reduction rate of around $70 \%-75 \%$ or higher allowed identification of the patients in whom the tumours were downstaged. ${ }^{10,11}$ Furthermore, a significant association with PCR was reported for patients with a volume reduction rate higher than $75 \% .{ }^{12}$ Recently, diffusion-weighted (DW) MR imaging after CRT was shown to be more valuable than morphologic MR imaging for the differentiation between a pCR and residual tumour, because on DW images, viable tumour remnants are more easily recognised, as they appear hyperintense compared with the low signal intensity (SI) of the surrounding nonneoplastic tissue. ${ }^{13,14}$ Hence, it can be hypothesised that volumetry of the tumour that is based on SI characteristics on DW images may be more accurate than conventional MR 
volumetry to distinguish between complete and noncomplete responders. In addition, promising results have been shown for quantitative DW imaging evaluation by measuring the apparent diffusion coefficient (ADC) for the evaluation of treatment response to CRT in patients with rectal cancer. ${ }^{15-21}$

With this study, we aim to determine the diagnostic performance of DW imaging for the assessment of a CR after CRT in patients with LARC by means of volumetric SI measurements and quantitative $A D C$ measurements and to compare the performance of DW imaging with volumetry on standard T2-weighted MR images.

\section{Materials \& Methods}

\section{Patients}

Eighty-six consecutive patients diagnosed with LARC at Maastricht University Medical Center (Maastricht, the Netherlands) between June 2006 and May 2010 were considered for inclusion in this retrospective study. Inclusion criteria consisted of (a) histopathologically (biopsy-) proved rectal adenocarcinoma; (b) locally advanced disease (staged on MR images as cT3-4 and/or N-category positive); (c) neoadjuvant treatment consisting of a long course of preoperative CRT (50.4 Gy radiation plus $2 \times 825 \mathrm{mg} / \mathrm{m}^{2} / \mathrm{d}$ capecitabine [Xeloda; Hoffmann-La Roche, Basel, Switzerland]); and (d) availability of pre- and post-CRT MR imaging results, including DW imaging results. Exclusion criteria were (a) nonresectable and/or metastatic disease and (b) insufficient MR image quality (eg, owing to metal implants or movement artifacts). Thirty-six patients were excluded for the following reasons: severe susceptibility artifacts on DW images owing to metal implants $(n=2)$, neoadjuvant treatment consisting of a short course of 5 Gy radiation on 5 consecutive days plus chemotherapy $(n=3)$, patient death during neoadjuvant treatment $(n=2)$, ineligibility to undergo surgery as a result of older age or comorbidity $(n=1)$, unresectable and/or metastatic disease $(n=9)$, or pre-CRT MR imaging performed without DW imaging $(n=19)$. This left a total of 50 patients (median age, 71.5 years; range, 51-90 years) who constituted the final study population. Thirty-six patients were men (median age, 70.5 years; range, 55-90 years) and 14 were women (median age, 76 years; range, 51-82 years). Clinical and imaging data were retrieved from a patient database originating from a previous imaging study approved by the local institutional ethical committee, for which all patients provided written informed consent. ${ }^{22}$ 


\section{MR imaging}

MR imaging was performed at $1.5 \mathrm{~T}$ (Intera; Philips Medical Systems, Best, the Netherlands) by using a phased-array body coil. All patients underwent pretreatment MR imaging for primary tumour staging and a second restaging MR imaging examination for response evaluation 6-8 weeks after completion of CRT. The imaging protocol consisted of the following: (a) standard two-dimensional T2weighted fast spin-echo sequences in three orthogonal directions (sagittal, coronal, axial) (repetition time msec/echo time $\mathrm{msec}$, 3427/150; flip angle, $90^{\circ}$; echo train length, 25 ; number of signals acquired, six; acquisition voxel size, $0.78 \times 1.14 \times 5.00$ $\mathrm{mm}$; sections, 22; acquisition time, 5.08 minutes) and (b) an axial DW sequence with background body signal suppression ${ }^{23}$ and $b$ values of $0,500,1000 \mathrm{sec} / \mathrm{mm}^{2}$ (4829/70; echo-planar imaging factor, 53; number of signals acquired, four; acquisition voxel size, $2.50 \times 3.11 \times 5.00 \mathrm{~mm}$; number of sections, 50 ; and acquisition time, 10.37 minutes). The axial T2-weighted and DW imaging sequences were used for volumetric analyses and were angled in identical planes, perpendicular to the tumour axis as defined on sagittal T2-weighted MR images. The coronal T2-weighted sequence was angled parallel to the tumour axis. Patients did not receive bowel preparation, antispasmodic medication, or rectal distention before any of the MR examinations.

\section{Volumetric image evaluation}

The MR images were evaluated on a picture archiving and communication system and were independently analysed by two observers, with 5 (L.C.) and 3 (D.M.J.L.) years of specific expertise in reading pelvic MR images. The observers were blinded to each other's results, the clinical patient data, and pathology reports. The readers calculated tumour volumes by manually tracing the tumour boundaries on the axial images and placing free-hand regions-of-interest (ROIs), which provided the sectional area of the lesion for each tumour-containing section (Figure 1). Wholetumour volume was then calculated by multiplying each cross-sectional area by section thickness. The DW and T2-weighted MR images were analysed independently and in random order, with a 1-week interval between the two reading sessions. On the $\mathrm{T} 2$-weighted images, tumour was defined as areas of isointense signal as compared with the relatively lower hypointense signal of the normal adjacent muscular rectal wall. On post-CRT T2-weighted MR images, areas of markedly low $\mathrm{SI}$ at the location of the primary tumour bed were interpreted as fibrosis. As the risk for residual tumour in these fibrotic areas is known to be $\pm 50 \%$, they were also included in the volumetric measurements. ${ }^{24}$ On the DW images, measurements were performed on high- $b$ value $\left(1000 \mathrm{sec} / \mathrm{mm}^{2}\right)$ images and were 
based on a visual analysis. Areas of high SI, compared with the normal bowel wall or background of lower SI tissue, were considered as tumour. For both data sets (T2 weighted and DW), the readers determined (a) pre-CRT tumour volume; (b) postCRT tumour volume; and (c) the tumour volume reduction ratio ( $\Delta$ volume), which was calculated as follows: $\left(\mathrm{TV}_{\text {pre }}-\mathrm{TV}_{\text {post }}\right) \times 100 / \mathrm{TV}_{\text {pre, }}$ where $\mathrm{TV}_{\text {pre }}$ is pre-CRT tumour volume and $\mathrm{TV}_{\text {post }}$ is post-CRT tumour volume.
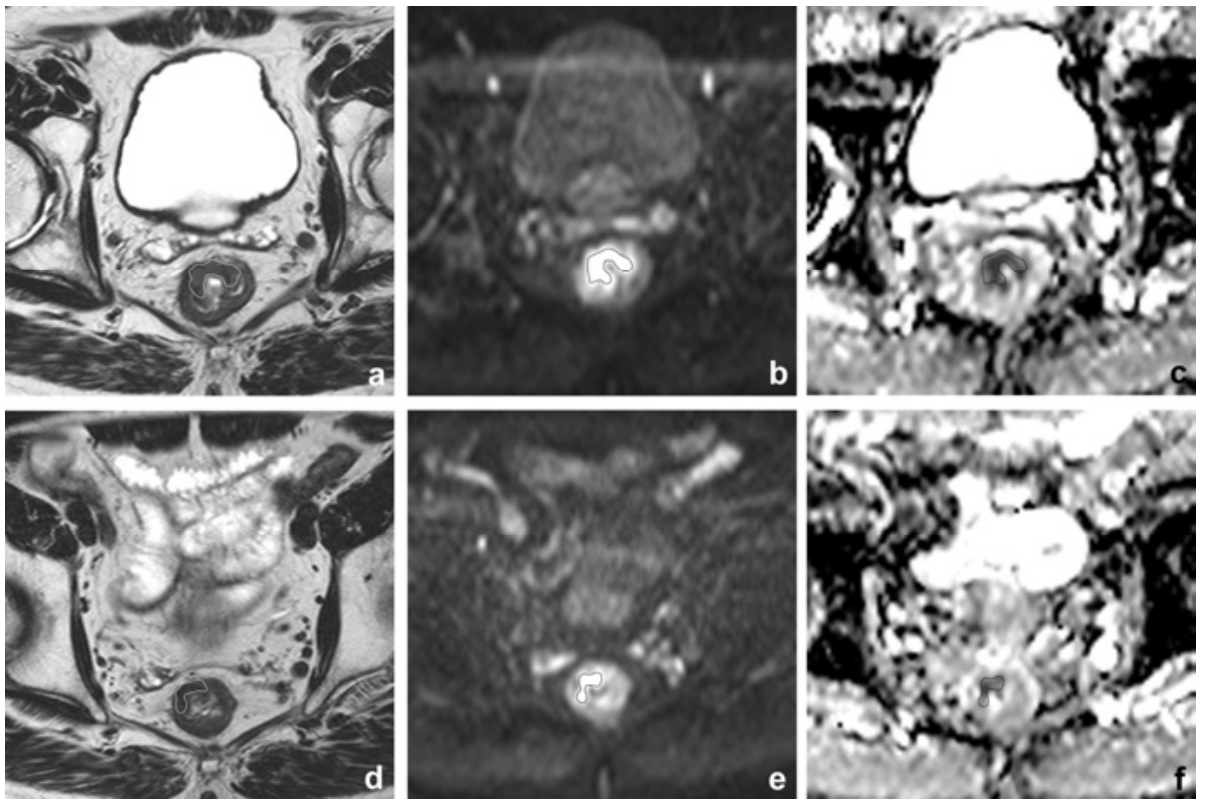

Figure 1. Examples of manual tracing of free-hand ROIs for calculation of the sectional area of tumour in each section performed on pre-CRT T2-weighted (a) and DW (b) MR images and on post-CRT T2weighted (d) and DW (e) MR images. ROIs were copied from the diffusion images with $b=1000 \mathrm{sec} / \mathrm{mm}^{2}$ to the corresponding pre-CRT (c) and post-CRT (f) ADC maps to calculate mean tumour ADC values. Sectional areas were multiplied by section thickness to determine the tumour volume.

\section{Measurement of the ADC}

The diffusion images were exported in Digital Imaging and Communications in Medicine format to an off-line MR workstation, on which ADC maps in gray scale were automatically generated by using a monoexponential decay model including all three $b$ values $\left(0,500\right.$, and $\left.1000 \mathrm{sec} / \mathrm{mm}^{2}\right)$. ROls covering the whole tumour volume (as described above) were reproduced on the MR operating system on the diffusion images with $b=1000 \mathrm{sec} / \mathrm{mm}^{2}$ by two independent readers (D.M.J.L. and T.T.), who were blinded to each other's results, the clinical patient data, and pathology reports. The ROls were then copied from the images with $b=1000$ 
$\mathrm{sec} / \mathrm{mm}^{2}$ to the ADC map to calculate mean pre- and post-CRT tumour ADCs (Figure 1). When no remaining high SI could be visualised on the post-CRT diffusion images, three sample measurements were obtained of the rectal wall at the former location of the primary tumour (Figure 2). In one patient, ADC measurements were not obtained, because his tumour had an entirely mucinous aspect (completely hyperintense in signal on T2-weighted MR images) without any solid tumour parts. These fully mucinous tumours are known to exhibit high ADC values, which would potentially lead to bias of the study results. ${ }^{25}$
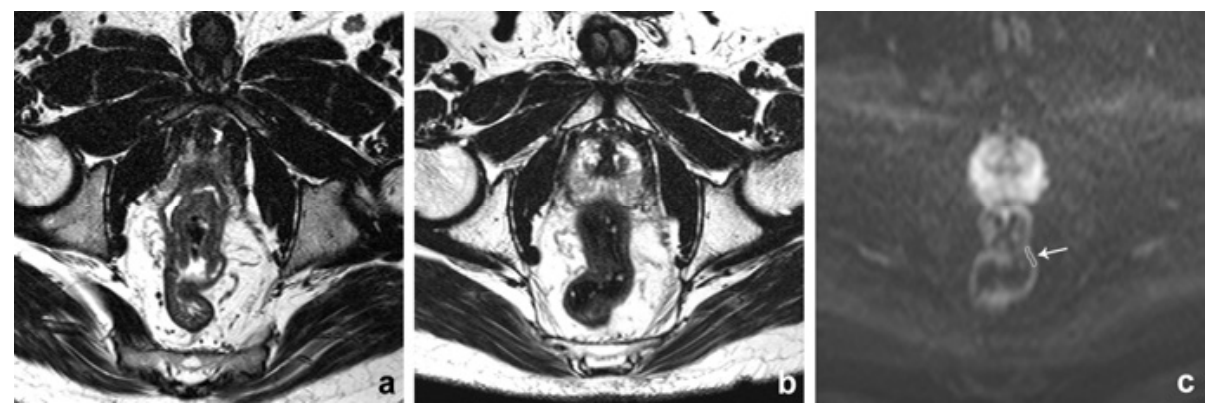

Figure 2. Interpretation of a CR on T2-weighted and DW MR images. (a) Pre-CRT DW image shows that there is a tumour visible in the distal rectum (arrows). (b) Post-CRT T2-weighted MR image shows that a normalised rectal wall is visualised. (c) High- $b$-value $\left(1000 \mathrm{sec} / \mathrm{mm}^{2}\right.$ ) post-CRT DW MR image shows absence of residual tumour, which was based on a lack of hyperintense SI areas of the bowel wall. To obtain post-CRT ADC measurements, three sample measurements (an example of which is indicated by the arrow) were taken from the normalised rectal wall at the location of the former tumour.

\section{Standard of reference}

Forty-two (84\%) patients underwent total mesorectal excision. The surgical resection specimens were histopathologically examined by dedicated pathologists experienced in colorectal cancer staging. Specimens were examined according to the Sixth American Joint Committee on Cancer TNM staging system. The tumour regression grade (TRG) was evaluated according to the method of Mandard et al. ${ }^{26}$ The response of the primary tumour was graded as follows: pCR ( $T$ stage after therapy at histopathologic evaluation, ypT0; TRG 1, no residual tumour cells) or residual tumour (ypT1-4; TRG 2-5, varying from rare residual cancer cells to a solid residual tumour mass). Eight (16\%) patients did not undergo surgery because of strong clinical evidence of a CR (repeated negative findings at sigmoidoscopy and biopsy after (RT). These patients underwent intensive 3-monthly follow-up, with a median local and distant recurrence-free follow-up period of 17 months, which was considered a surrogate end point for a CR. 


\section{Statistical analyses}

Statistical analyses were performed by using software (SPSS, version 16.0; SPSS, Chicago, III). Interobserver variability for the three readings (T2-weighted MR volumetry, DW MR volumetry, and ADC), as well as the correlation between the volume measurements on T2-weighted and DW images were analysed by calculating the intraclass correlation coefficient (ICC) for single measurements (0-0.20, poor correlation; 0.21-0.40, fair correlation; 0.41-0.60, moderate correlation; 0.61-0.80, good correlation; and 0.81-1.00, excellent correlation). Volumes and ADCs were averaged between the two observers for further analysis. A Student $t$ test was used to compare the mean ADCs between the complete responders and noncomplete responders. As the tumour volumes were not normally distributed, a Mann-Whitney $U$ test was used to compare the tumour volumes between the complete responder and noncomplete responder groups. $A$ Wilcoxon signed-rank test was used to compare pre- and post-CRT volumes, and a paired-samples $t$ test was used to compare the pre- and post-CRT ADC measurements. Receiver operating characteristic (ROC) curves were generated to evaluate the diagnostic performance in detecting a CR for (a) T2-weighted MR volumetry, (b) DW MR volumetry, and (c) ADC. Corresponding areas under the ROC curve (AUCs), sensitivities, specificities, positive predictive values, and negative predictive values were calculated. For these analyses, cutoff values were determined according to the point nearest to the upper left corner in the ROC curves. Differences in diagnostic performance were analysed by comparing the ROC curves according to the method described by DeLong et al. ${ }^{27} \mathrm{~A}$ difference with a $P$ value of less than 0.05 was considered significant.

\section{Results}

\section{Patient and treatment characteristics}

Twenty-four patients underwent a low anterior resection, 12 underwent an abdominoperineal resection, and six underwent an extended resection. Histopathologic analysis of the surgical specimen yielded the following findings: Six patients had ypT0, four had ypT1, 13 had ypT2, 18 had ypT3, and one had ypT4 tumour. Six patients had mucinous type adenocarcinoma. The median time between the restaging MR imaging and surgery was 20 days (range, 4-197 days). Together with the eight nonsurgically treated patients, the total number of patients with a CR corresponded to 14 (28\%). 


\section{Interobserver agreement}

The interobserver agreement (ICC) for the T2-weighted MR volume measurements was 0.93 on pre-CRT MR images and 0.79 on post-CRT MR images. For the DW MR volume measurements, ICCs were 0.96 on pre-CRT MR images versus 0.75 on postCRT MR images. For the ADC measurements, ICCs were 0.91 on pre-CRT MR images versus 0.61 on post-CRT MR images. Figure 3 illustrates the effect of interobserver variations on the volumetric DW MR and ADC measurements.
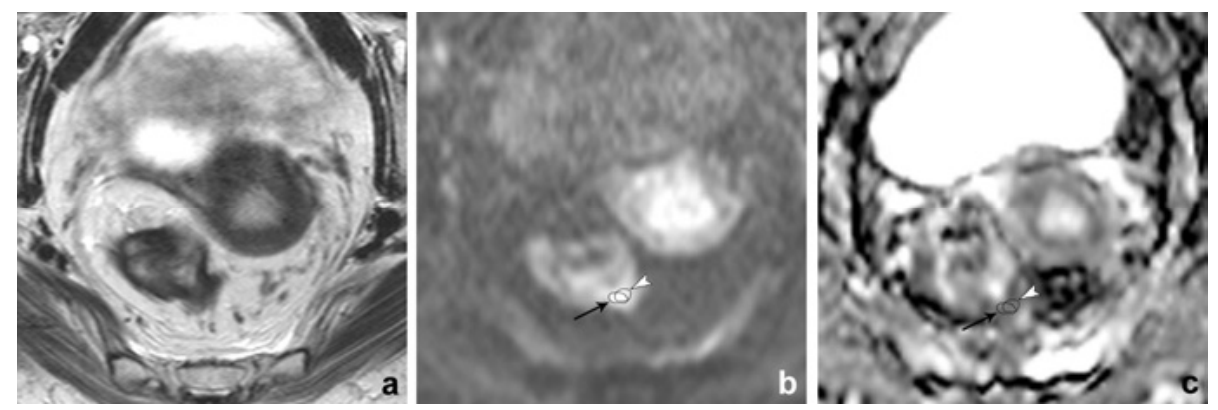

Figure 3. Post-CRT images in 79-year-old female patient with a ypT3 residual tumour. (a) T2-weighted MR image shows persistent wall thickening suggestive of residual tumour (intermediate $\mathrm{SI}=$ arrowheads) and fibrosis (low $\mathrm{SI}=$ arrow). (b) DW MR image shows a small focus of hyperintensity in the bowel wall. To delineate the tumour volume, the ROI was placed as shown by observers 1 (arrow, also on c) and 2 (arrowhead, also on c). The two ROls resulted in comparable tumour areas (0.48 vs $0.46 \mathrm{~cm}^{2}$ ). (c) ADC map derived from the DW images shows that corresponding ADC values were 1.21 versus $1.36 \times 10^{-3}$ $\mathrm{mm}^{2} / \mathrm{sec}$ for the ROls placed by the two respective observers. The difference in ADC between the two observers $\left(0.15 \times 10^{-3} \mathrm{~mm}^{2} / \mathrm{sec}\right)$ for this single measurement was thus larger than the overall difference in mean tumour ADC observed between the complete responder and noncomplete responder groups, which was $0.07 \times 10^{-3} \mathrm{~mm}^{2} / \mathrm{sec}$ for the post-CRT measurements. This example illustrates that small variations in $\mathrm{ROI}$ size and/or placement may result in nonnegligible variations in ADC that may substantially influence study results.

\section{T2-weighted versus DWI volumetry}

The median tumour volumes from T2-weighted MR and DW MR images (mean of two observers) for the whole patient group and for the complete responder versus noncomplete responder groups are displayed in Table 1. Median tumour volumes decreased from 21.8 to $5.5 \mathrm{~cm}^{3}$ on T2-weighted MR images $(P<0.001)$ and from 18.0 to $1.0 \mathrm{~cm}^{3}$ on DW MR images $(P<0.001)$. There were no significant differences in pre-CRT volumes between the complete responder and noncomplete responder groups on neither T2-weighted MR images (20.6 vs $24.3 \mathrm{~cm}^{3}, P=0.46$ ) nor DW MR images (11.9 vs $18.9 \mathrm{~cm}^{3}, P=0.16$ ). The post-CRT volumes were significantly smaller for the complete responder group compared with the noncomplete responder group, both on T2-weighted MR images (1.2 vs $5.6 \mathrm{~cm}^{3}, P=0.03$ ) and DW MR 
images $\left(0.03\right.$ vs $\left.1.5 \mathrm{~cm}^{3}, P<0.001\right)$. The $\Delta$ volume was significantly larger for the complete responder group than it was for the noncomplete responder group, both on T2-weighted MR images (-92.2 vs $-78.1 \%, P<0.001)$ and DW MR images (-99.7 vs $-90.2 \%, P<0.001$ ). The correlation (ICC) between the volume measurements derived from T2-weighted and DW MR images was 0.97 for the pre-CRT measurements and 0.25 for post-CRT measurements.

\begin{tabular}{|c|c|c|c|c|c|c|c|}
\hline \multicolumn{5}{|c|}{ Table 1. Median volumes and mean ADC values } \\
\hline
\end{tabular}

\section{Tumour ADC values}

The mean tumour ADC values for the whole patient group and for the complete responder versus noncomplete responder groups are displayed in Table 1 . Mean tumour ADC for the two observers increased from $1.09 \times 10^{-3} \mathrm{~mm}^{2} / \mathrm{sec}$ on pre-CRT MR images to $1.43 \times 10^{-3} \mathrm{~mm}^{2} / \mathrm{sec}$ on post-CRT MR images $(P<0.001)$. There were no significant differences in pre-CRT, post-CRT, or $\triangle A D C$ between the complete responder and noncomplete responder groups $(P=0.61,0.48$ and 0.96 , respectively). 
Table 2.

Diagnostic performance for volume measurements (from T2W and DWI) and ADC in detecting a CR

\begin{tabular}{|c|c|c|c|c|c|c|c|}
\hline & & Sensitivity & Specificity & PPV & NPV & Accuracy & $\begin{array}{l}\text { Optimal } \\
\text { Cut-Off }\end{array}$ \\
\hline \multirow{6}{*}{$\begin{array}{l}\text { Pre- } \\
\text { CRT }\end{array}$} & $\mathrm{T} 2 \mathrm{~W}$ & $43(6 / 14)$ & $83(30 / 36)$ & $50(6 / 12)$ & $79(30 / 38)$ & $72(36 / 50)$ & \multirow{2}{*}{$16.7 \mathrm{~cm}^{3}$} \\
\hline & $95 \% \mathrm{Cl}$ & $17-71$ & $67-93$ & $21-78$ & $62-90$ & $57-83$ & \\
\hline & DWI & $57(8 / 14)$ & $78(28 / 36)$ & $50(8 / 16)$ & $82(28 / 34)$ & $72(36 / 50)$ & \multirow{2}{*}{$12.5 \mathrm{~cm}^{3}$} \\
\hline & $95 \% \mathrm{Cl}$ & $28-82$ & $60-89$ & $24-75$ & $65-93$ & $57-83$ & \\
\hline & $A D C$ & $38(5 / 13)$ & $81(29 / 36)$ & $42(5 / 12)$ & $78(29 / 37)$ & $69(34 / 49)$ & \multirow{2}{*}{$\begin{array}{c}0.97 * 10^{-3} \\
\mathrm{~mm}^{2} / \mathrm{s}\end{array}$} \\
\hline & $95 \% \mathrm{Cl}$ & $13-68$ & $63-91$ & $15-72$ & $61-90$ & $54-81$ & \\
\hline \multirow{6}{*}{$\begin{array}{l}\text { Post- } \\
\text { CRT }\end{array}$} & $\mathrm{T} 2 \mathrm{~W}$ & $64(9 / 14)$ & $94(34 / 36)$ & $82(9 / 11)$ & $87(34 / 39)$ & $86(43 / 50)$ & \multirow{2}{*}{$1.6 \mathrm{~cm}^{3}$} \\
\hline & $95 \% \mathrm{Cl}$ & $35-87$ & $81-99$ & $48-97$ & $72-95$ & $73-94$ & \\
\hline & DWI & $79(11 / 14)$ & $100(36 / 36)$ & $100(11 / 11)$ & $92(36 / 39)$ & $94(47 / 50)$ & \multirow{2}{*}{$0.15 \mathrm{~cm}^{3}$} \\
\hline & $95 \% \mathrm{Cl}$ & 49-95 & $90-100$ & $71-100$ & $79-98$ & $83-98$ & \\
\hline & $A D C$ & $46(6 / 13)$ & $56(20 / 36)$ & $27(6 / 22)$ & $74(20 / 27)$ & $53(26 / 49)$ & \multirow{2}{*}{$\begin{array}{c}1.41 * 10^{-3} \\
\mathrm{~mm}^{2} / \mathrm{s}\end{array}$} \\
\hline & $95 \% \mathrm{Cl}$ & $19-74$ & $38-72$ & $10-50$ & $53-88$ & $38-67$ & \\
\hline \multirow{6}{*}{$\begin{array}{l}\text { Delta } \\
(\%)\end{array}$} & $\mathrm{T} 2 \mathrm{~W}$ & $79(11 / 14)$ & $92(33 / 36)$ & 79 (11/14) & $92(33 / 36)$ & $88(44 / 50)$ & \multirow{2}{*}{$-88.6 \%$} \\
\hline & $95 \% \mathrm{Cl}$ & $49-95$ & $77-98$ & $49-95$ & $77-98$ & $75-95$ & \\
\hline & DWI & $86(12 / 14)$ & $89(32 / 36)$ & $75(12 / 16)$ & $94(32 / 34)$ & $88(44 / 50)$ & \multirow{2}{*}{$-97.5 \%$} \\
\hline & $95 \% \mathrm{Cl}$ & $57-98$ & $73-96$ & $47-92$ & $80-99$ & $75-95$ & \\
\hline & $A D C$ & $54(7 / 13)$ & $64(23 / 36)$ & $35(7 / 20)$ & $79(23 / 29)$ & $61(30 / 49)$ & \multirow{2}{*}{$+25.3 \%$} \\
\hline & $95 \% \mathrm{Cl}$ & $25-80$ & $46-79$ & $15-59$ & $60-92$ & $46-74$ & \\
\hline
\end{tabular}

Numbers are percentages. Absolute numbers are given in parentheses. Cut-off values were chosen according to the point nearest to the upper left corner in the ROC curves.

$\mathrm{PPV}=$ positive predictive value, $\mathrm{NPV}=$ negative predictive value, $95 \% \mathrm{Cl}=95 \%$ confidence interval.

\section{Diagnostic performance for assessment of CR}

The ROC curves used to compare the diagnostic performance of the pre-CRT, post$\mathrm{CRT}$, and $\Delta$ measurements of T2-weighted MR volumetry, DW MR volumetry, and $\mathrm{ADC}$ for assessment of a $\mathrm{CR}$ are shown in Figure 4. Corresponding accuracy data are provided in Table 2. The pre-CRT measurements resulted in AUCs of 0.57, 0.63, and 0.55 for T2-weighted MR volumetry, DW MR volumetry, and ADC, respectively, which were not significantly different from each other $(P=0.15-0.85)$. For the postCRT measurements, AUCs were 0.70 for T2-weighted MR volumetry, 0.93 for DW MR volumetry, and 0.54 for ADC. The results for DW MR volumetry were 
significantly better compared with either T2-weighted MR volumetry or ADC $(P=0.02$ and $P<0.001$, respectively). The $\Delta$ volumes of T2-weighted MR (AUC, 0.84) and DW MR (AUC, 0.92) were significantly better than $\triangle \mathrm{ADC}$ (AUC, $0.51 ; P=0.003$ and $P<0.001$, respectively). The difference in AUC between the $\Delta$ volumes from T2weighted and DW MR was not significant $(P=0.42)$. The performance of post-CRT DW MR volumetry was equally accurate as the $\Delta$ volumes from T2-weighted $(P=0.31)$ and DW $(P=0.65) \mathrm{MR}$.
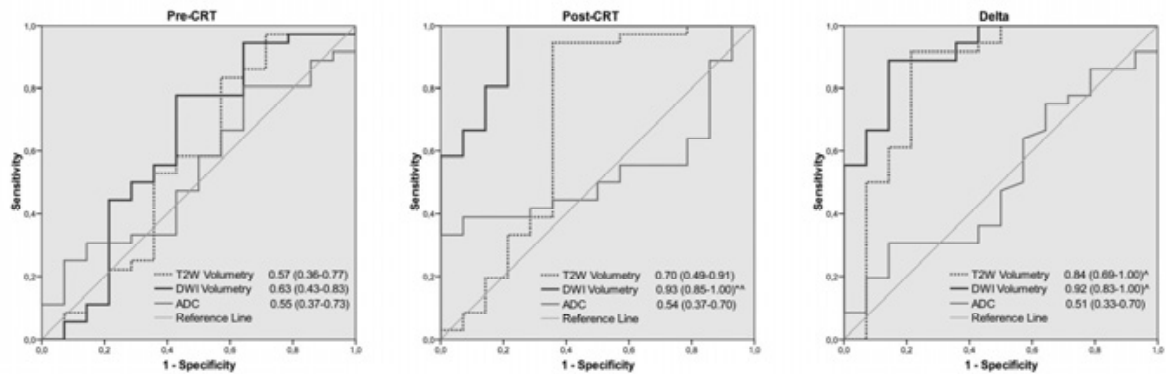

Figure 4. Comparison of ROC curves displaying the diagnostic performance for pre-and post-CRT volumes and $\Delta$ volume (Delta) on T2- and DW MR images and ADC in the assessment of a CR. Numbers = AUC values for each sequence, numbers in parentheses $=95 \%$ confidence intervals, ${ }^{*}=$ significant difference in AUC compared with T2-weighted MR volumetry, and ${ }^{\wedge}=$ significant difference in AUC compared with ADC.

\section{Interpretation errors on DWI}

Eleven of the 14 complete responders could be detected on the basis of the absence of high SI on DW MR images. The three false-negative findings on DW MR images were caused by the following reasons: In one patient, high SI caused by a collapsed rectal wall at the location of the primary tumour was erroneously interpreted as residual tumour. In the other two patients, DW MR images showed small areas of high SI at the location of the primary tumour, while histopathologic findings indicated mainly fibrosis, necrosis, and inflammation without any residual tumour cells (Figure 5). 

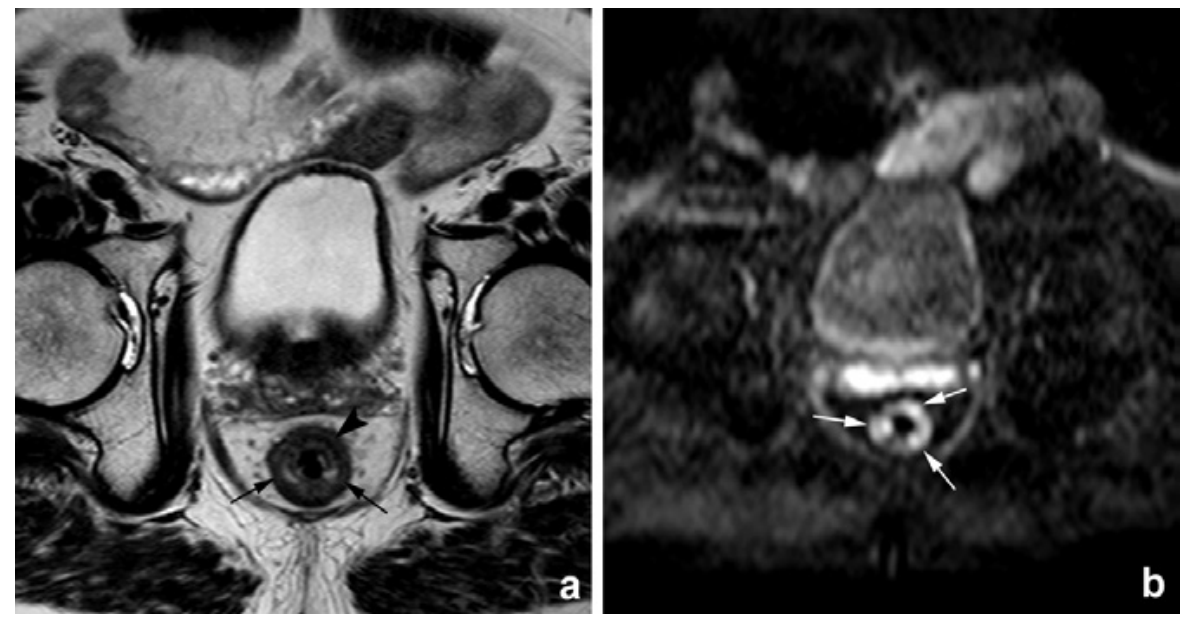

Figure 5. (a) Post-CRT T2-weighted and (b) DW MR images in 71-year-old male patient with a CR in whom the presence of residual tumour was overestimated by both observers on both images. T2weighted MR image demonstrates a persistent wall thickening with predominant low SI owing to fibrosis (arrowhead), but also with some areas of intermediate SI interpreted as residual tumour (arrows). On b, hyperintense areas (arrows) were interpreted as residual tumour within the bowel wall. Histopathologic examination revealed no viable neoplastic tissue (ypT0), although some nonneoplastic epithelial tissue of unknown origin was found within the muscular layer, which may explain the difficulties in interpreting the images.

\section{Discussion}

The goal of our study was to evaluate the diagnostic performance of DW MR imaging for the assessment of a complete tumour response in patients with LARC and to compare it with tumour volume measurements on conventional T2-weighted MR images. For this purpose, DW MR images were evaluated in twofold: (a) by volume measurements of high $\mathrm{SI}$ areas on diffusion images with $b=1000 \mathrm{sec} / \mathrm{mm}^{2}$ and (b) by measurement of tumour ADC values. The results of our study demonstrate that post-CRT DW MR volumetry provides high diagnostic performance (AUC, 0.93) for the assessment of a $C R$ and is significantly more accurate than is post-CRT T2-weighted MR volumetry (AUC, 0.70 ) or post-CRT ADC (AUC, 0.54). The post-CRT DW MR volumetry was equal in performance to volume reduction measurements ( $\triangle$ volume) of both T2-weighted and DW MR (AUC, 0.84 and 0.92, respectively). Pre-CRT DW MR and T2-weighted MR volumetry, as well as $A D C$, were not reliable to identify a $C R$, with AUCs ranging between 0.51 and 0.63 .

Previously published data addressed the value of rectal tumour volumetry on standard T2-weighted MR images for the assessment of response after CRT and showed conflicting results. Kang et al $^{12}$ reported a significant association with $\mathrm{pCR}$ 
for patients with a tumour volume reduction rate of more than $75 \%$. Kim et $\mathrm{al}^{28}$ could not confirm these findings and showed no difference in the tumour volume reduction rates between patients with $\mathrm{PCR}$ and those with residual disease. The findings of our study showed that volume reduction measurements performed either on T2-weighted MR images or diffusion images with $b=1000 \mathrm{sec} / \mathrm{mm}^{2}$ can be used to assess a CR, with an overall accuracy of $88 \%$ for both techniques. Of interest, however, was our finding that tumour volumetry performed on post-CRT DW MR images only was equal in performance to the tumour volume reduction measurements on T2-weighted and DW MR images, suggesting that evaluation of pre-CRT images may not even be necessary. The latter is further supported by the fact that, in our study, pretreatment measurements were not reliable for the assessment of a CR.

Another interesting finding was that volumetry on post-CRT DW MR images was significantly more accurate than that on post-CRT T2-weighted MR images for assessing a CR. Apparently, the tumour volumes measured on the basis of the presence (or absence) of high-SI areas on DW MR images better represented the actual presence of residual tumour. On morphologic post-CRT MR images, it is more difficult to measure volumes because it is difficult to define which of the fibrotic areas are still suspicious for tumour and should be included in the volume measurements and which should not. We experienced that those difficulties were less pronounced on DW MR images and that, on DW MR images, the delineation of residual tumour was more clear-cut. This was also reflected in the poor correlation (ICC, 0.25 ) between the post-CRT tumour volumes measured on T2-weighted and DW MR images.

Given the high diagnostic performance of post-CRT DW MR volumetry on the basis of signal perception on images with $b=1000 \mathrm{sec} / \mathrm{mm}^{2}$, it could be hypothesised that a visual evaluation of whether or not a high SI suggestive of residual tumour is remaining will be sufficient, and volumetric measurements are not even required. Such a visual approach would also be more practical and far less time consuming. Previous authors ${ }^{13,14}$ have already shown good results for a visual analysis of DW MR images. In these reports, the value of adding DW MR imaging to standard MR imaging was assessed by evaluating the DW and T2-weighted MR images side by side and comparing findings of this evaluation with those on T2weighted MR images only. Kim et al $^{13}$ reported an AUC of $0.82-0.88$ for the combined reading of standard MR images plus DW MR images, results comparable to those of Lambregts et al $^{14}$ who reported AUCs ranging between 0.78 and 0.80 for three independent readers. In our study, we found an even higher AUC (0.93) for the assessment of a CR with DW MR imaging. A possible explanation could be that, in our study, we evaluated the DW MR images independently from T2-weighted MR 
images and with objective volume measurements, whereas in the above-mentioned works, T2-weighted and DW MR images were read side by side and by means of subjective interpretation. For example, if a radiologist has already determined a strong suspicion of residual tumour on the basis of the T2-weighted MR image morphologic findings, he or she will not be eager to alter the diagnosis even if the DW MR image would show the contrary. This factor, together with the knowledge that, in oncology, one should better err on the "safe" side and, in case of doubt, should best diagnose a patient as having residual disease than to potentially incorrectly categorise that patient as having a $C R$, might have incorporated some bias in the evaluation of DW MR images in published literature. In our study, this bias was eliminated, since the definition of a pCR on a DW MR image was solely based on the absolute absence of hyperintense areas within the rectal wall and the DW MR images were evaluated independently from the T2-weighted images.

Despite our favorable results for DW MR imaging, we acknowledge that it remains difficult to differentiate between patients with a CR (TRG 1 ) and patients with small microscopic clusters of residual tumour (TRG 2). Furthermore, it is difficult to obtain a precise correlation between DW MR imaging findings and the underlying histopathologic findings at a microscopic level. Further studies are required to address this issue.

In our study, we failed to demonstrate a benefit for pre-CRT ADC, post-CRT $A D C$, or $\triangle A D C$ measurements to differentiate between patients with a $C R$ and residual tumour. A possible explanation could be that $A D C$ measurements are more subject to measuring errors, because of the inherently low discriminatory power and lesion conspicuity on ADC images. Even subtle variations in ROI size and ROI positioning between two readers may result in substantial variations in ADC. We believe that this phenomenon significantly contributed to the low performance of $A D C$ in our study to precisely distinguish between complete and noncomplete responders. This factor is less an issue when the response groups are more roughly categorised in "responding" and "nonresponding" patient groups, as was done by a number of previous authors. ${ }^{16-20}$ Obviously, such large subcategories will require less precise discrimination methods and is the reason why we believe that these published data have shown more favorable results for ADC.

There were some limitations to our study design. First, eight of 14 complete responders were classified in a group with a wait-and-see approach and histopathologic findings were only available from the biopsy specimen. It should be stressed, however, that these patients are classified in a group with a very strict follow-up protocol, including regular (3-monthly) clinical, endoscopic (with biopsy), and imaging examinations and that, to this date (at 17 months of follow-up), none have developed recurrent disease. A second issue is that a proportion of the 
included patients had relatively small tumours, which can be explained by the current trend in our institution to stratify an increasing number of small rectal cancers into the pre-CRT regimen. Finally, we acknowledge that, ideally, our study design should also have included an evaluation of intraobserver variability. This was, however, not practically feasible owing to the highly time-consuming methods required to obtain the volumetric and ADC measurements.

In conclusion, post-CRT volumetry on DW MR images was significantly more accurate than was post-CRT volumetry on T2-weighted MR images to assess a CR after CRT in patients with LARC. Post-CRT DW MR was equally as accurate as volume reduction ( $\Delta$ volume) measurements on either DW or T2-weighted MR images. PreCRT volume measurements were not accurate. The above findings suggest that evaluation of post-CRT DW MR images can be sufficient and pre-CRT images do not necessarily have to be evaluated. ADC measurements were not reliable for the assessment of a CR. 


\section{References}

1. Sauer R, Becker H, Hohenberger W, et al. Preoperative versus postoperative chemoradiotherapy for rectal cancer. N Engl J Med 2004; 351:1731-1740.

2. Maas M, Nelemans PJ, Valentini V, et al. Long-term outcome in patients with a pathological complete response after chemoradiation for rectal cancer: a pooled analysis of individual patient data. Lancet Oncol 2010; 11:835-844.

3. Habr-Gama A, Perez RO, Proscurshim I, et al. Patterns of failure and survival for nonoperative treatment of stage c0 distal rectal cancer following neoadjuvant chemoradiation therapy. J Gastrointest Surg 2006; 10:1319-1328; discussion 1328-1319.

4. Bujko K, Kepka L, Nowacki MP. Chemoradiotherapy alone for rectal cancer: a word of caution. Lancet Oncol 2007; 8:860-862; author reply 862-863.

5. O'Neill BD, Brown G, Heald RJ, Cunningham D, Tait DM. Chemoradiotherapy alone for rectal cancer: a word of caution-author's reply [Letter]. Lancet Oncol 2007; 8:662-863.

6. O'Neill BD, Brown G, Heald RJ, Cunningham D, Tait DM. Non-operative treatment after neoadjuvant chemoradiotherapy for rectal cancer. Lancet Oncol 2007; 8:625-633.

7. Chen CC, Lee RC, Lin JK, Wang LW, Yang SH. How accurate is magnetic resonance imaging in restaging rectal cancer in patients receiving preoperative combined chemoradiotherapy? Dis Colon Rectum 2005; 48:722-728.

8. Huh JW, Park YA, Jung EJ, Lee KY, Sohn SK. Accuracy of endorectal ultrasonography and computed tomography for restaging rectal cancer after preoperative chemoradiation. J Am Coll Surg 2008; 207:7-12.

9. Barbaro B, Vitale R, Leccisotti L, et al. Restaging locally advanced rectal cancer with MR imaging after chemoradiation therapy. Radiographics 2010; 30:699-716.

10. Dresen RC, Beets $\mathrm{GL}$, Rutten $\mathrm{HJ}$, et al. Locally advanced rectal cancer: MR imaging for restaging after neoadjuvant radiation therapy with concomitant chemotherapy. Part I. Are we able to predict tumor confined to the rectal wall? Radiology 2009; 252:71-80.

11. Barbaro B, Fiorucci C, Tebala C, et al. Locally advanced rectal cancer: MR imaging in prediction of response after preoperative chemotherapy and radiation therapy. Radiology 2009; 250:730-739.

12. Kang JH, Kim YC, Kim H, et al. Tumor volume changes assessed by three-dimensional magnetic resonance volumetry in rectal cancer patients after preoperative chemoradiation: the impact of the volume reduction ratio on the prediction of pathologic complete response. Int J Radiat Oncol Biol Phys 2010; 76:1018-1025.

13. Kim SH, Lee JM, Hong SH, et al. Locally advanced rectal cancer: added value of diffusion-weighted MR imaging in the evaluation of tumor response to neoadjuvant chemo- and radiation therapy. Radiology 2009; 253:116-125.

14. Lambregts DMJ, Vandecaveye V, Barbaro B, et al. Diffusion-weighted MRI for selection of complete responders after chemoradiation for locally advanced rectal cancer: a multicenter study. Ann Surg Oncol 2011; 18:2224-31.

15. Kim SH, Lee JY, Lee JM, Han JK, Choi BI. Apparent diffusion coefficient for evaluating tumour response to neoadjuvant chemoradiation therapy for locally advanced rectal cancer. Eur Radiol 2011; 21:987-95.

16. Hein PA, Kremser C, Judmaier W, et al. Diffusion-weighted magnetic resonance imaging for monitoring diffusion changes in rectal carcinoma during combined, preoperative chemoradiation: preliminary results of a prospective study. Eur J Radiol 2003; 45:214-222.

17. DeVries AF, Kremser C, Hein PA, et al. Tumor microcirculation and diffusion predict therapy outcome for primary rectal carcinoma. Int J Radiat Oncol Biol Phys 2003; 56:958-965.

18. Kremser C, Judmaier W, Hein P, Griebel J, Lukas P, de Vries A. Preliminary results on the influence of chemoradiation on apparent diffusion coefficients of primary rectal carcinoma measured by magnetic resonance imaging. Strahlenther Onkol 2003; 179:641-649. 
19. Sun YS, Zhang XP, Tang L, et al. Locally advanced rectal carcinoma treated with preoperative chemotherapy and radiation therapy: preliminary analysis of diffusion-weighted MR imaging for early detection of tumor histopathologic downstaging. Radiology 2010; 254:170-178.

20. Dzik-Jurasz A, Domenig C, George M, et al. Diffusion MRI for prediction of response of rectal cancer to chemoradiation. Lancet 2002; 360:307-308.

21. Roth $\mathrm{Y}$, Tichler $\mathrm{T}$, Kostenich $\mathrm{G}$, et al. High-b-value diffusion-weighted MR imaging for pretreatment prediction and early monitoring of tumor response to therapy in mice. Radiology 2004; 232:685-692.

22. Lambregts DM, Beets GL, Maas M, et al. Accuracy of Gadofosveset-enhanced MRI for Nodal Staging and Restaging in Rectal Cancer. Ann Surg 2011; 253:539-545.

23. Takahara T, Imai Y, Yamashita T, Yasuda S, Nasu S, Van Cauteren M. Diffusion weighted whole body imaging with background body signal suppression (DWIBS): technical improvement using free breathing, STIR and high resolution 3D display. Radiat Med 2004; 22:275-282.

24. Vliegen RF, Beets GL, Lammering G, et al. Mesorectal fascia invasion after neoadjuvant chemotherapy and radiation therapy for locally advanced rectal cancer: accuracy of MR imaging for prediction. Radiology 2008; 246:454-462.

25. Woodhams R, Kakita S, Hata $\mathrm{H}$, et al. Diffusion-weighted imaging of mucinous carcinoma of the breast: evaluation of apparent diffusion coefficient and signal intensity in correlation with histologic findings. AJR Am J Roentgenol 2009; 193:260-266.

26. Mandard AM, Dalibard F, Mandard JC, et al. Pathologic assessment of tumor regression after preoperative chemoradiotherapy of esophageal carcinoma. Clinicopathologic correlations. Cancer 1994; 73:2680-2686.

27. DeLong ER, DeLong DM, Clarke-Pearson DL. Comparing the areas under two or more correlated receiver operating characteristic curves: a nonparametric approach. Biometrics 1988; 44:837-845.

28. Kim YH, Kim DY, Kim TH, et al. Usefulness of magnetic resonance volumetric evaluation in predicting response to preoperative concurrent chemoradiotherapy in patients with resectable rectal cancer. Int J Radiat Oncol Biol Phys 2005; 62:761-768. 

TUMOUR ADC MEASUREMENTS IN RECTAL CANCER: EFFECT OF ROI METHODS ON ADC VALUES AND INTEROBSERVER VARIABILITY

\section{CHAPTER}

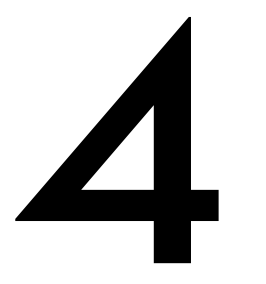

DMJ Lambregts GL Beets M Maas

L Curvo-Semedo AGH Kessels T Thywissen RGH Beets-Tan

Published in: European Radiology 2011; 21(12):2567-2574 


\section{Abstract}

\section{Aim}

To assess the influence of region of interest (ROI) size and positioning on tumour $A D C$ measurements and interobserver variability in patients with locally advanced rectal cancer (LARC).

\section{Materials \& Methods}

Forty-six LARC patients were retrospectively included. Patients underwent MRI including DWI (b0,500,1000) before and 6-8 weeks after chemoradiation (CRT). Two readers measured mean tumour ADCs (pre- and post-CRT) according to three ROI protocols: whole-volume, single-slice or small solid samples. The three protocols were compared for differences in $A D C, S D$ and interobserver variability (measured as the intraclass correlation coefficient; ICC).

\section{Results}

ICC for the whole-volume ROIs was excellent (0.91) pre-CRT versus good (0.66) postCRT. ICCs were 0.53 and 0.42 for the single-slice ROIs versus 0.60 and 0.65 for the sample ROIs. Pre-CRT ADCs for the sample ROIs were significantly lower than the whole-volume or single-slice ROIs. Post-CRT there were no significant differences among the three ROI methods. The SDs for the whole-volume and single-slice ROIs were significantly larger than for the sample ROls.

\section{Conclusions}

ROI size and positioning have a considerable influence on tumour ADC values and interobserver variability. Interobserver variability is worse after CRT. ADCs obtained from the whole tumour volume provide the most reproducible results. 


\section{Introduction}

At present, the standard treatment for patients with locally advanced rectal cancer consists of a long course of neoadjuvant chemoradiation treatment (CRT) followed by surgical resection. As surgery is routinely performed in each patient - regardless of the response to treatment - response evaluation after CRT has so far not been a major issue. Nowadays there is, however, a trend towards minimally invasive treatments instead of standard surgery for well-responding patients. ${ }^{1-3}$. Accurate response assessment then becomes relevant, as it may directly influence treatment planning. ${ }^{18}$ F-Fluorodeoxyglucose-Positron Emission Tomography (FDG-PET) and MRI have been most extensively studied for response evaluation, but these techniques suffer from limitations in the interpretation of fibrotic scar tissue and inflammation. ${ }^{4,5}$ Diffusion-weighted MR Imaging (DWI) is a functional imaging technique that analyses differences in the extracellular movement of water protons to discriminate between tissues of varying cellularity. ${ }^{6}$ Different publications on DWI have shown its potentially beneficial role for the detection and characterisation of malignant tumours. ${ }^{7-9}$ In addition, changes in tumour diffusion during and after treatment are indicative of tissue changes on a cellular level and may be used to evaluate treatment response. ${ }^{10,11}$ Previous studies in a variety of tumour types have suggested that quantitative interpretation of the apparent diffusion coefficient (ADC) can be used as a biomarker for response to treatment. ${ }^{12-15}$ For rectal cancer patients specifically, a benefit for treatment response evaluation by measuring tumour ADC values before ${ }^{16-19}$, during ${ }^{16-18,20,21}$, and after chemoradiation treatment has been suggested. ${ }^{22,23}$ Nevertheless - as also previously pointed out in a review by Patterson et al. $^{10}$ - there is no consensus yet on the true clinical value of ADC measurements for response assessment in rectal cancer. This is because the available literature consists of mainly small-scale studies with conflicting results. Moreover, in most studies, DWI evaluation was only performed by a single reader and $A D C$ measurements by a variety of methods for region of interest (ROI) placement. Whereas some authors included the whole tumour volume ${ }^{17-19,22,24}$, others included only a single tumour slice ${ }^{16,21}$ or small tumour samples ${ }^{23}$, which may contribute to the large variety in reported ADC results. It remains unclear whether ROIs for ADC measurements should ideally incorporate the entire tumour volume or only a representative tumour section. Furthermore, none of the studies focusing on rectal tumour ADC have addressed the issue of interobserver variability, which is a non-negligible factor when considering the use of $A D C$ as a potential marker for response in clinical practice.

The purpose of the current study is to assess the influence of ROI sizes and positioning in tumour $A D C$ measurements on interobserver variability and $A D C$ 
values before and after chemoradiation treatment in patients with locally advanced rectal cancer. We aim to determine which method provides the most reproducible results in order to provide a reference for further studies.

\section{Materials \& Methods}

\section{Patients}

This study retrospectively evaluated 46 patients who were treated for locally advanced rectal cancer between 2006 and 2010. Clinical patient data were retrieved from a patient database originating from a previous imaging study approved by the local institutional review board, for which the patients provided written informed consent. Thirty-four patients were male and 12 were female. Median age was 70 years (range 49-88). Inclusion criteria consisted of (a) histologically (biopsy) proven rectal adenocarcinoma, (b) locally advanced disease, defined on primary staging T2weighted MRI by an experienced gastrointestinal radiologist as tumour in the distal rectum ( $\leq 5 \mathrm{~mm}$ from the anorectal junction), threatened or involved circumferential resection margins $(\leq 2 \mathrm{~mm}$ margin between the tumour and mesorectal fascia) and/or positive nodal stage ( $\geq 1$ suspicious nodes, i.e. $>5 \mathrm{~mm}$ in size and/or heterogeneous signal intensity and/or irregular border), (c) treatment consisting of a long course of preoperative CRT (50.4 Gy radiation $+2 \times 825$ $\mathrm{mg} / \mathrm{m}^{2} / \mathrm{d}$ capecitabine) followed by surgical resection and (d) availability of pre- and post-CRT MR imaging including DWI. Patients with non-resectable and/or metastatic disease were excluded. Mucinous tumours are known to have a very low cellular density and will therefore exhibit high $A D C$ values. ${ }^{25}$ As this may bias the study results, patients with predominantly mucinous appearing tumours (identified as predominantly high signal lesions on T2-weighted MRI) were also excluded.

\section{MR imaging}

Patients did not receive bowel preparation or spasmolytics. Imaging was performed at 1.5T (Intera; Philips Medical Systems, Best, The Netherlands) using a phased array body coil. All patients underwent a pre-treatment MRI for primary tumour staging and a second, restaging MRI for response evaluation 6-8 weeks after completion of CRT. The imaging protocol consisted of standard 2D T2-weighted (T2W) fast spinecho sequences (FSE) in three orthogonal directions and an axial DWI with a singleshot echo planar imaging sequence, according to the method of diffusion-weighted sequence with background body signal suppression (DWIBS), acquired with b-values of 0,500 and $1000 \mathrm{~s} / \mathrm{mm}^{2}{ }^{26}$ The sequence parameters are displayed in Table 1 . The 
axial T2W and DWI sequences were angled in identical planes and were planned perpendicular to the tumour axis as defined on sagittal MRI. ADC maps in greyscale were automatically generated at the operating system, using a monoexponential decay model including all three b-values.

Table 1. Sequence parameters

\begin{tabular}{|l|c|c|}
\hline & T2W FSE & DWI* \\
\hline Repetition time (ms) & $3427-8456$ & 4829 \\
\hline Echo time (ms) & $130-150$ & 1 \\
\hline Echotrain length & 25 & $2.50 \times 3.11$ \\
\hline In plane resolution (mm x mm) & $0.78 \times 1.14$ & 5 \\
\hline Section thickness (mm) & $3-5$ & -1 \\
\hline Section gap (mm) & 2 & 50 \\
\hline No. of sections & $22-30$ & 4 \\
\hline No. of signals acquired & 6 & 2 \\
\hline Sensitivity encoding (SENSE) factor & - & 53 \\
\hline Echo planar imaging (EPI) factor & - & 10.37 \\
\hline Acquisition time (min) & $5.08-6.03$ & \\
\hline
\end{tabular}

* DWI was acquired with b-values of 0,500 and $1000 \mathrm{~s} / \mathrm{mm}^{2}$

\section{Image evaluation}

The MR images were independently analysed by two radiological researchers (DMJL and TT), who performed tumour ADC measurements on the pre- and postchemoradiation images. The readers were blinded to each other's results, the clinical patient data and pathology reports. Mean tumour ADC was evaluated by manually drawing regions of interest (ROI) on the high b-value (b1000) diffusion images and copying them to the corresponding ADC map (Figure 1). The mean ADC + standard deviation (SD) and the number of pixels per ROI was recorded for each individual measurement. On the pre-treatment b1000 diffusion images, tumour was defined as a focal mass showing high signal intensity compared with the signal of the normal adjacent rectal wall and corresponding with the tumour (mass showing intermediate signal intensity) on the anatomical T2-weighted MRI. On the postchemoradiation DWI, tumour was defined as focal areas of residual high signal on the b1000 images within the location of the primary tumour bed and/or 

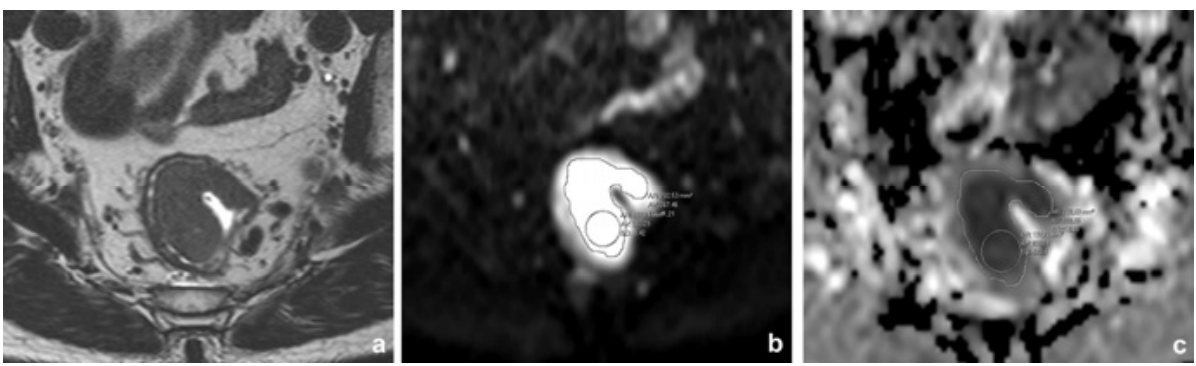

Figure 1. Axial T2-weighted image (a), b1000 diffusion image (b) and ADC map (c) of a male patient with a tumour in the rectum. For the whole-volume and single-slice methods, ADC was measured by drawing freehand ROIs along the high signal intensity border of the tumour on the b1000 images (b) to cover the entire tumour area. ROIs were copied to the ADC map (c) to calculate ADC. For the solid sample method, tumour ADC was measured by drawing three oval- or round-shaped ROls within the most solid tumour areas.
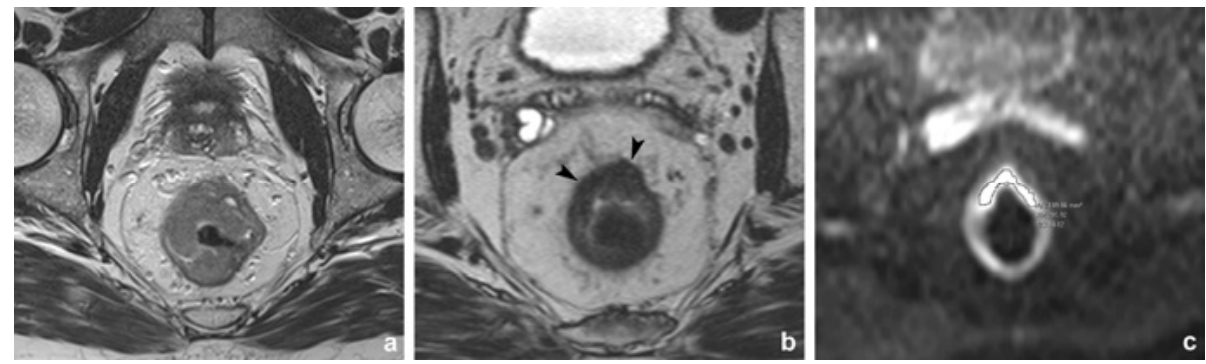

Figure 2. Axial pre- (a) and post-treatment (b) T2-weighted images of a male patient with a rectal tumour. After treatment, the tumour has undergone mainly fibrotic changes (arrowheads). On the corresponding b1000 diffusion image, an ROI was drawn along a well-defined area of high signal intensity within the fibrosis, suggestive of residual tumour. At histology, a residual ypT2 tumour was found
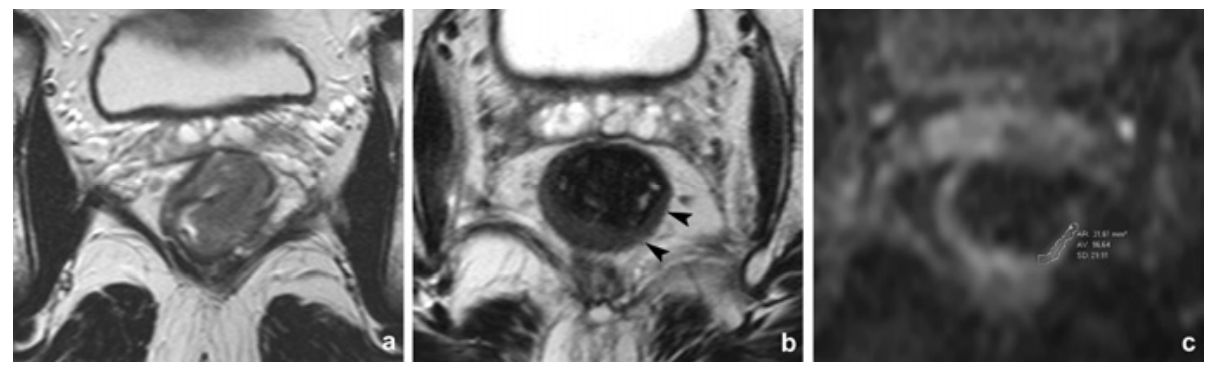

Figure 3. Axial T2-weighted images of a male patient with a rectal tumour before (a) and after (b) chemoradiation treatment. After CRT, the rectal wall has normalised (arrowheads). On the corresponding b1000 diffusion image (c), no high signal was observed and ROIs were placed within the rectal wall at the location of the primary tumour to measure post-treatment ADC. At histology, the patient had undergone a complete response 
corresponding with residual tumour on T2-weighted MRI (Figure 2). The pretreatment images were at the readers' disposal when analysing the post-treatment images, in order to compare and identify the location of the tumour. When no remaining high signal could be visualised on DWI, three sample measurements were obtained of the rectal wall at the former location of the primary tumour, one of which is illustrated in Figure 3.

\section{ROI protocols}

Mean tumour ADCs were measured according two three distinct ROI protocols: (a) 'Whole-volume', (b) 'Single-slice' and (c) 'Solid tumour samples'. For the whole volume method, freehand ROIs were drawn along the border of the high signal of the tumour on the b1000 images to cover the entire tumour area of each consecutive tumour-containing slice. Mean ADC (+ SD) was obtained for each slice and $A D C$ values were averaged to calculate the mean $A D C$ of the whole tumour volume. For the single-slice method, a single freehand ROI was drawn in the same way (along the border of the tumour), but only on a single slice containing the largest available tumour area. For the third method, mean ADC was calculated from a sample of three round/oval-shaped ROls that were placed within the most solid tumour part (as identified on T2W-MRI) of three independent tumour-containing slices, an example of which is illustrated in Figure 1.

\section{Statistical analyses}

Statistical analyses were performed using the Statistical Package for the Social Sciences (SPSS, version 16.0, Inc., Chicago, IL, USA). Interobserver variability for the tumour ADC measurements of the two readers for the pre- and post-CRT ADC measurements and for each individual ROI method was analysed according to the method of Bland and Altman and by calculating the intraclass correlation coefficient (0.00-0.20 poor, 0.21-0.40 fair, 0.41-0.60 moderate, 0.61-0.80 fair and 0.81-1.00 excellent correlation). ADCs were averaged between the two observers for further analyses. A paired samples $t$-test was used to compare (a) the pre- and posttreatment $A D C s$ and $(b)$ the tumour $A D C$ values obtained by the three different ROI methods. For each patient, the average variance was calculated over the different slice measurements, weighted with the number of pixels. The mean SD for each patient was calculated as the square root of the variance. The variance (mean for the whole patient group) of the different ROI measurement methods and for the pre- and post-CRT measurements was compared using the F-statistics with the total number of slices as the degree of freedom. $P$ values $<0.05$ were considered statistically significant. 


\section{Results}

\section{Patient and treatment characteristics}

Twenty-seven patients underwent a low anterior resection, 15 an abdominoperineal resection and 4 more extended surgery. At histology 6 patients had a ypT0, 5 ypT1, 14 ypT2, 20 ypT3 and 1 a ypT4 status. Thirty-three patients had a ypN0, 9 ypN1 and 4 ypN2 status.

\section{Table 2. Influence of choice of regions of interest (ROIs)}

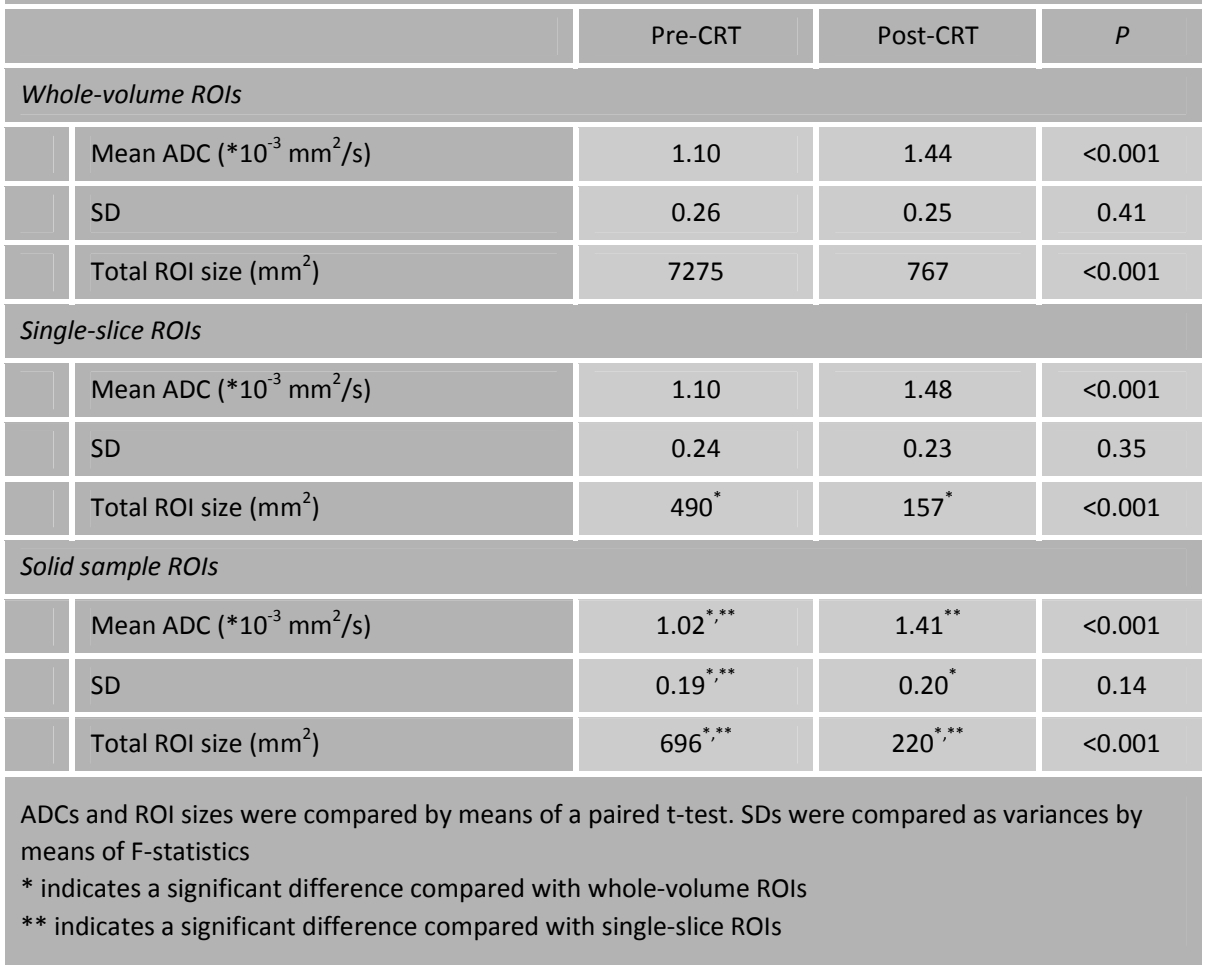

\section{Effect of ROI methods}

The mean tumour ADCs, SDs and total ROI sizes are displayed in Table 2 for the preand post-treatment measurements of each respective ROI protocol. Mean pretreatment tumour ADC was significantly lower when measured by means of small sample ROls, compared with the whole-volume $(P<0.001)$ or single-slice protocol $(P<0.001)$, respectively. For the post-CRT measurements there were no significant differences in tumour ADC between the whole-volume ROIs compared with the 
single-slice $(P=0.07)$ or small sample ROls $(P=0.08)$, respectively, but the singleslice ROIs resulted in significantly higher ADCs compared with the small sample ROls $(P=0.002)$. For the pre-CRT measurements, the variance (SD) of the small sample ROI measurements was significantly smaller than for the whole-volume ROIs $(P<0.001)$ and single-slice ROIs $(P=0.03)$, respectively. For the post-CRT measurements, the variance of the small sample ROls was also smaller than that of the whole-volume ROIs $(P=0.003)$ and single-slice ROIs, although the latter difference was not statistically significant $(P=0.06)$. There were no significant differences in tumour ADC or variance between the whole-volume and single-slice approaches.

Table 3. Interobserver variability (measured as the intraclass correlation coefficient*) for the different ROI protocols

\begin{tabular}{|l|c|c|}
\hline & Pre-CRT & Post-CRT \\
\hline Whole-volume ROIs & 0.91 & 0.66 \\
\hline Single-slice ROIs & 0.53 & 0.42 \\
\hline Solid sample ROls & 0.60 & 0.65 \\
\hline
\end{tabular}

* 0.00-0.20 poor; 0.21-0.40 fair; 0.41-0.60 moderate; 0.61-0.80 good; 0.81-1.00 excellent correlation
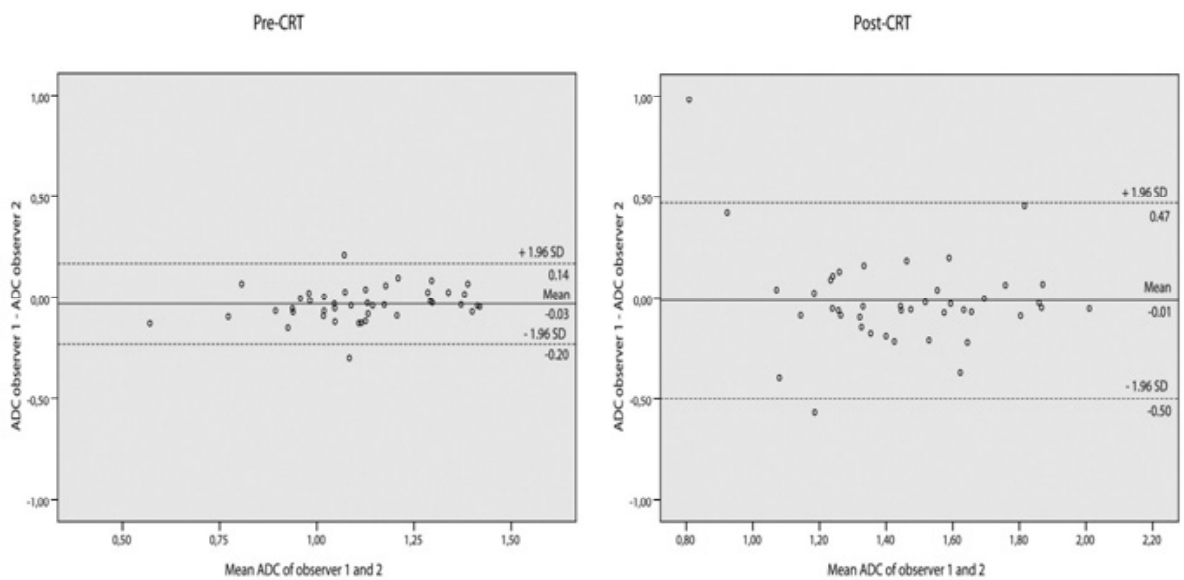

Figure 4. Interobserver reproducibility for the whole-volume tumour ADC measurements performed preand post-chemoradiation treatment. Bland-Altman plots of the mean ADC of the two observers ( $x$-axis) against the difference in ADC between the two observers ( $y$-axis). The continuous lines represent the mean absolute difference (bias) in ADC between the two observers; the dashed lines represent the $95 \%$ confidence intervals of the mean differences (limits of agreement) 


\section{Interobserver variability}

Intraclass correlation coefficients between the two readers are provided in Table 3 for the three ROI protocols. The interobserver reproducibility was excellent (ICC 0.91) for the pre-CRT whole-volume ADC measurements, and good (ICC 0.66) for the post-CRT measurements. For the single-slice and solid sample ROls, the ICCS ranged from 0.42 to 0.65 . Figure 4 displays the Bland-Altman plots for the wholevolume measurements performed pre- and post-CRT.

\section{Discussion}

The results of this study show that when measuring ADC in patients with locally advanced rectal cancer, tumour ADC values and interobserver variability are highly dependent on methods of ROI analysis. ADC measurements obtained from the whole tumour volume are more reproducible than those obtained from single-slice or small sample measurements. In specific pre-treatment whole-volume ADC measurements result in excellent interobserver reproducibility.
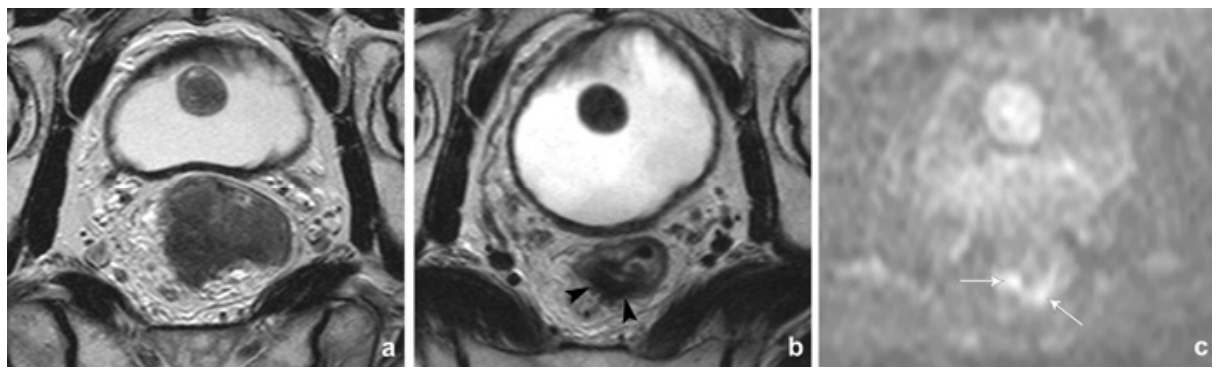

Figure 5. Axial T2-weighted images of a male patient with a rectal tumour before (a) and after (b) chemoradiation treatment. An ill-defined residual area of hypointense signal intensity, indicative of fibrosis, is visible after CRT (arrowheads). On the corresponding diffusion image (c) there is still an area of high signal intensity, suggestive of residual tumour (arrows). Because of its irregular aspect and illdefined borders, however, it is difficult to delineate an ROI, explaining the relatively poor interobserver agreement for the post-CRT ADC measurements. At histology, a ypT1 residual tumour was found

The number and size of the ROls affected the interobserver agreement. When comparing the different ROI protocols, the single-slice or sample ROIs resulted in considerably poorer interobserver agreement (ICC 0.42-0.65) than the whole-volume ROIs (ICC 0.66-0.91), indicating that analysing a larger number of pixels results in more reproducible ADC values. Interobserver agreement for the whole-volume ADC measurements before treatment was excellent (ICC 0.91), but results after treatment were poorer (ICC 0.66). After chemoradiation, rectal 
tumours have often undergone massive fibrotic changes and defining a region of tumour residue within the fibrosis may be more difficult (Figure 5). In cases where the tumour has completely regressed and the bowel wall has normalised or become fibrotically thickened, it can be even more challenging to correctly define a ROI (Figure 3). After CRT, ADC measurements thus seem to be more affected by the interpretation skills of the reader than before CRT, when the tumour is generally better defined.

The choice of ROIs also significantly influenced the tumour ADC values. On pre-CRT MRI, the whole-volume and single-slice ROls resulted in significantly higher tumour ADC values than the small sample ROIs. The small sample ROIs only included the most viable solid tumour parts, which may explain the lower ADC values. In this setting, areas of necrosis are likely to be excluded from the ADC measurements, while the presence of necrosis before onset of treatment is in fact believed to be an important indicator when aiming at evaluating response. A previous study of Roth and co-authors showed that whole-volume tumour ADC measurements were a better predictor of response than ROls chosen only from viable regions of the tumour. ${ }^{18}$ Although the focus in their study was on perfusion CT in patients with colorectal cancer, Goh et al. also found that when obtaining pharmacokinetic parameters by applying different ROI sizes and positions, whole tumour volume measurements were the most reliable. ${ }^{27}$ The above-described phenomenon may also explain why the whole-volume ADC measurements resulted in a larger variance and higher standard deviations, which is likely to reflect the heterogeneous nature of the tumour, including solid foci, as well as areas of necrosis and fibrosis. Altogether these findings suggest that whole-volume measurements might be a better indicator of tumour viability and may therefore be more suitable for assessment of response. Furthermore, as was also stressed by Goh et $\mathrm{al}^{27}$, if variations in ROI substantially influence the measurements, efforts should be made to standardise their application for clinical use. Interestingly, we observed no significant differences in tumour ADC or SD between the whole-volume measurements and the single-slice approach, suggesting that the latter may also be used as a less time-consuming alternative. However, one should keep in mind that the single-slice method was subject to a much larger interobserver variability and whole-volume measurements thus remain the single most reliable method.

Our study is limited because of its retrospective nature and the relatively small patient numbers. Furthermore, it was sometimes difficult to position regions of interests due to susceptibility artefacts occurring around air-tissue interfaces. This was especially challenging after chemoradiation, in cases where only limited or no residual tumour could be identified on DWI. Susceptibility artefacts might be minimised by applying rectal wall distension with intraluminal filling, which we have 
not done in the current study. The specific focus of this study was to determine the effect of ROI size and positioning on tumour ADC evaluation and not to assess the relation between $A D C$ and response, as various previous authors have done. ${ }^{16-24}$ As such, we chose not to include a correlation between $A D C$ and histopathological parameters of response.

In conclusion, variations in ROI size and positioning have a significant effect on tumour $A D C$ values and interobserver variability. The most reproducible results are obtained when measuring $A D C$ of the whole tumour volume. Interobserver variability is larger after chemoradiation treatment than before. These issues should be taken into account when considering the use of ADC as a potential biomarker for response in clinical practice. 


\section{References}

1. Habr-Gama A, Perez RO, Proscurshim I, Canpos FG, Nadalin W, Kiss D, Gama-Rodrigues J. Patterns of failure and survival for nonoperative treatment of stage $\mathrm{c} 0$ distal rectal cancer following neoadjuvant chemoradiation therapy. J Gastrointest Surg 2006;10:1319-1328; discussion 1328-1319

2. Lezoche G, Baldarelli M, Guerrieri M, Paganini AM, De Sanctis A, Bartolacci S, Lezoche E. A prospective randomized study with a 5-year minimum follow-up evaluation of transanal endoscopic microsurgery versus laparoscopic total mesorectal excision after neoadjuvant therapy. Surg Endosc 1008;22:352-358.

3. Maas M, Nelemans PJ, Valentini V, et al. Long-term outcome in patients with a pathological complete response after chemoradiation for rectal cancer: a pooled analysis of individual patient data. Lancet Oncol 2010;11:835-844.

4. Barbaro B, Vitale R, Leccisotti L, et al. Restaging locally advanced rectal cancer with MR imaging after chemoradiation therapy. Radiographics 2010; 30:699-716.

5. Capirci C, Rubello D, Chierichetti F, et al. Restaging after neoadjuvant chemoradiotherapy for rectal adenocarcinoma: role of F18-FDG PET. Biomed Pharmacother 2004; 58:451-457.

6. Bammer R. Basic principles of diffusion-weighted imaging. Eur J Radiol 2003; 45(3):169-184.

7. Koh DM, Collins DJ. Diffusion-weighted MRI in the body: applications and challenges in oncology. AJR Am J Roentgenol 2007;188:1622-1635.

8. Bruegel M, Holzapfel K, Gaa J, et al. Characterization of focal liver lesions by ADC measurements using a respiratory triggered diffusion-weighted single-shot echo-planar MR imaging technique. Eur Radiol 2008;18:477-485

9. Lim HK, Kim JK, Kim KA, Cho KS. Prostate cancer: apparent diffusion coefficient map with T2weighted images for detection-a multireader study. Radiology 2009;250:145-151

10. Patterson DM, Padhani AR, Collins DJ. Technology insight: water diffusion MRI--a potential new biomarker of response to cancer therapy. Nat Clin Pract Oncol 2008;5:220-233.

11. Padhani AR, Liu G, Koh DM, et al. Diffusion-weighted magnetic resonance imaging as a cancer biomarker: consensus and recommendations. Neoplasia 2009;11:102-125.

12. Theilmann RJ, Borders R, Trouard TP, et al. Changes in water mobility measured by diffusion MRI predict response of metastatic breast cancer to chemotherapy. Neoplasia 2004;6:831-837.

13. Cui Y, Zhang XP, Sun YS, Tang L, Shen L. Apparent diffusion coefficient: potential imaging biomarker for prediction and early detection of response to chemotherapy in hepatic metastases. Radiology 2008;248:894-900.

14. Koh DM, Scurr E, Collins D, Kanber B, Norman A, Leach MU, Husband JE. Predicting response of colorectal hepatic metastasis: value of pretreatment apparent diffusion coefficients. AJR Am J Roentgenol 2007;188:1001-1008.

15. Jain R, Scarpace LM, Ellika S, Torcuator R, Schultz LR, Hearshen D, Mikkelsen T. Imaging response criteria for recurrent gliomas treated with bevacizumab: role of diffusion weighted imaging as an imaging biomarker. J Neurooncol 2010;96:423-431.

16. Dzik-Jurasz A, Domenig C, George M, Wolber J, Padhani A, Brown G, Doran S. Diffusion MRI for prediction of response of rectal cancer to chemoradiation. Lancet 2002;360:307-308.

17. Sun YS, Zhang XP, Tang L, Ji JF, Gu J, Cai Y, Zhang XY. Locally advanced rectal carcinoma treated with preoperative chemotherapy and radiation therapy: preliminary analysis of diffusion-weighted $M R$ imaging for early detection of tumor histopathologic downstaging. Radiology 2010;254:170-178.

18. Roth $\mathrm{Y}$, Tichler $\mathrm{T}$, Kostenich $\mathrm{G}$, et al. High-b-value diffusion-weighted MR imaging for pretreatment prediction and early monitoring of tumor response to therapy in mice. Radiology 2004;232:685-692.

19. Lambrecht M, Deroose $C$, Roels $S$, et al. The use of FDG-PET/CT and diffusion-weighted magnetic resonance imaging for response prediction before, during and after preoperative chemoradiotherapy for rectal cancer. Acta Oncol 2010;49:956-63. 
20. Kremser C, Judmaier W, Hein P, Griebel J, Lukas P, de Vries A. Preliminary results on the influence of chemoradiation on apparent diffusion coefficients of primary rectal carcinoma measured by magnetic resonance imaging. Strahlenther Onkol 2003;179:641-649.

21. Hein PA, Kremser C, Judmaier $W$, et al. Diffusion-weighted magnetic resonance imaging for monitoring diffusion changes in rectal carcinoma during combined, preoperative chemoradiation: preliminary results of a prospective study. Eur J Radiol 2003;45:214-222.

22. Kim SH, Lee JY, Lee JM, Han JK, Choi BI. Apparent diffusion coefficient for evaluating tumour response to neoadjuvant chemoradiation therapy for locally advanced rectal cancer. Eur Radiol 2011;21:987-995

23. Kim SH, Lee JM, Hong SH, Kim GH, Lee JY, Han JK, Choi BI. Locally advanced rectal cancer: added value of diffusion-weighted MR imaging in the evaluation of tumor response to neoadjuvant chemoand radiation therapy. Radiology 2009;253:116-125.

24. Seierstad T, Roe K, Olsen DR. Noninvasive monitoring of radiation-induced treatment response using proton magnetic resonance spectroscopy and diffusion-weighted magnetic resonance imaging in a colorectal tumor model. Radiother Oncol 2007;85:187-194.

25. Woodhams R, Kakita S, Hata H, Iwabuchi K, Umeoka S, Mountford CE, Hatabu H. Diffusion-weighted imaging of mucinous carcinoma of the breast: evaluation of apparent diffusion coefficient and signal intensity in correlation with histologic findings. AJR Am J Roentgenol 2009;193:260-266.

26. Takahara T, Imai Y, Yamashita T, Yasuda S, Nasu S, Van Cauteren M. Diffusion weighted whole body imaging with background body signal suppression (DWIBS): technical improvement using free breathing, STIR and high resolution 3D display. Radiat Med 2004;22:275-282.

27. Goh V, Halligan S, Gharpuray A, Wellsted D, Sundin J, Bartram Cl. Quantitative assessment of colorectal cancer tumor vascular parameters by using perfusion CT: influence of tumor region of interest. Radiology 2008;247:726-732. 
LYMPH NODES 

VALUE OF ADC MEASUREMENTS FOR NODAL STAGING AFTER CHEMORADIATION IN LOCALLY ADVANCED RECTAL CANCER - A PER LESION VALIDATION STUDY

\section{CHAPTER}

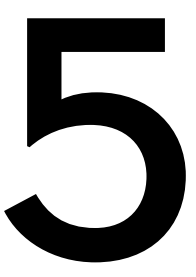

DMJ Lambregts M Maas RG Riedl FCH Bakers JL Verwoerd AGH Kessels G Lammering C Boetes GL Beets RGH Beets-Tan

Published in: European Radiology 2011; 21(2):265-273 


\section{Abstract}

\section{Aim}

To evaluate the performance of diffusion-weighted MRI (DWI) in addition to T2weighted (T2W) MRI for nodal restaging after chemoradiation in rectal cancer.

\section{Materials \& Methods}

Thirty patients underwent chemoradiation followed by MRI (1.5 T) and surgery. Imaging consisted of T2W-MRI and DWI (b0, 500, 1000). On T2W-MRI, nodes were scored as benign/malignant by two independent readers (R1, R2). Mean apparent diffusion coefficient $(A D C)$ was measured for each node. Diagnostic performance was compared for T2W-MRI, ADC and T2W+ADC, using a per lesion histological validation.

\section{Results}

ADC was higher for the malignant nodes $\left(1.43 \pm 0.38\right.$ vs $1.19 \pm 0.27 * 10^{-3} \mathrm{~mm}^{2} / \mathrm{s}$, $\mathrm{p}<0.001$ ). Area under the ROC curve/sensitivity/specificity were $0.88 / 65 \% / 93 \%$ (R1) and $0.95 / 71 \% / 91 \%$ (R2) using T2W-MRI; 0.66/53\%/82\% using ADC (mean of two readers); and $0.91 / 56 \% / 98 \%$ (R1) and $0.96 / 56 \% / 99 \%$ (R2) using $T 2 W+A D C$. There was no significant difference between T2W-MRI and T2W+ADC. Interobserver reproducibility was good for T2W-MRI $(\kappa \quad 0.73)$ and ADC (intraclass correlation coefficient 0.77 ).

\section{Conclusions}

After chemoradiation, ADC measurements may have potential for nodal characterisation, but DWI on its own is not reliable. Addition of DWI to T2W-MRI does not improve accuracy and T2W-MRI is already sufficiently accurate. 


\section{Introduction}

Nodal involvement constitutes one of the most significant risk factors for local recurrence in rectal cancer. ${ }^{1}$ For patients with node-positive disease the standard treatment consists of a long course of chemoradiation therapy (CRT), followed by surgery. In up to $35 \%$ of patients, no residual tumour cells are found at histological evaluation after surgery. ${ }^{2,3}$. These complete responders are known to have a good clinical outcome, with respect to local control and survival. ${ }^{4,5}$ Therefore, it is now increasingly being suggested that minimally invasive treatments, such as a local excision or a wait-and-see policy be advocated as an alternative to standard surgery with the aim of reducing treatment-related morbidity and mortality. ${ }^{6,7}$ When doing so it is, however, mandatory to ensure that no metastatic nodes are left behind and that the true node-negative (the ypNO) patients are accurately selected. CT, endoluminal ultrasound and MRI are all known to be insufficiently accurate with sensitivities and specificities in the $55-78 \%$ range, although some authors have reported more encouraging results after $\mathrm{CRT}$. $^{8-11}$

Diffusion-weighted MRI (DWI) could be a potentially valuable oncological imaging technique. DWI uses differences in water motion to discriminate between tissues of varying cellularity. As lymph nodes have a high cellular density, they generally show restricted diffusion and are easily detected on DWI. ${ }^{12,13}$. Quantification of the apparent diffusion coefficient (ADC) could be useful to discriminate between benign and metastatic nodes, as the cellular density - and therefore the diffusion - is likely to alter when nodes are invaded with tumour. Only a few studies, mainly in head/neck and uterine/cervical cancer, have focussed on DWI for lymph node characterisation and most showed only a per patient or per region validation. ${ }^{13-23}$ To our knowledge there have been no reports so far of a per node DWI validation study in rectal cancer and in particular focussing on nodal restaging after CRT.

This study therefore aims to assess the diagnostic value of diffusionweighted MRI for nodal staging in rectal cancer patients undergoing preoperative chemoradiation therapy, using a lesion-by-lesion histological validation as the reference standard. A second aim is to evaluate the interobserver reproducibility for $A D C$ measurements of rectal cancer nodes. 


\section{Materials and methods}

\section{Patients}

Between April 2008 and December 2009, 40 consecutive locally advanced rectal cancer patients were considered for inclusion in this prospective study. The study was part of a nodal imaging study, approved by the local institutional review board, for which all patients provided written informed consent. Inclusion criteria consisted of (a) biopsy-proven rectal adenocarcinoma, (b) locally advanced disease, defined on primary staging T2W-MRI as tumour in the distal rectum $(\leq 5 \mathrm{~mm}$ from the anorectal junction), tumour involvement of the circumferential resection margins and/or positive nodal stage, and (c) treatment consisting of CRT + resection. Ten patients were excluded; 3 did not undergo surgery, in 2 patients the MR images showed severe metal implant artefacts and in 5 patients there was no $\mathrm{PhD}$ fellow present during histopathological evaluation so that a lesion-by-lesion matching could not be performed. This left a total of 30 patients ( 25 male, 5 female; median age 71 years, range 47-90) who were included in the study. The CRT consisted of $28 \times 1.8 \mathrm{~Gy}$ and $2 \times 825 \mathrm{mg} / \mathrm{m}^{2} / \mathrm{d}$ capecitabine, followed by a restaging MRI 6-8 weeks after completion of CRT and subsequent surgery.

\section{MR imaging}

Patients did not receive a bowel preparation or spasmolytics. Imaging was performed with a 1.5-T MR unit (Intera; Philips Medical Systems, Best, The Netherlands) using a a phased-array body coil. The imaging protocol consisted of standard 2D T2-weighted (T2W) fast spin-echo sequences in three orthogonal directions (TR/TE $3427 / 150 \mathrm{msec}, 90^{\circ}$ flip angle, 25 echotrain length, 6 NSA, $0.78 x$ $1.14 \times 5.00 \mathrm{~mm}$ acquisition voxel size, 22 slices, 5.08 minutes acquisition time). For study purposes, two additional sequences were performed; [a] an axial diffusionweighted sequence with background body signal suppression (DWIBS ${ }^{24}$, b-values 0,500,1000 s/mm ${ }^{2}$, TR/TE 4829/70 msec, EPI factor 53, 4 NSA, $2.50 \times 3.11 \times 5.00 \mathrm{~mm}$ acquisition voxel size, 50 slices, 10.37 minutes acquisition time) and (b) an axial 3DT1W gradient-echo (GRE) sequence (TR/TE 9.8/4.6 msec, $15^{\circ}$ flip angle, 1 NSA, $1.15 \times 1.15 \times 1.00 \mathrm{~mm}$ acquisition voxel size, 200 slices, 6.30 minutes acquisition time). The 3DT1W GRE sequence is not part of a standard rectal MRI protocol, but was used as a roadmap to depict small lymph nodes and confirm them anatomically as nodes and not vessels. 


\section{Image evaluation}

The MR images were independently analysed by two readers: a senior (RGHBT) and junior (FCHB) gastrointestinal radiologist with 13 and 3 years of respective expertise in reading pelvic MRI. The readers were blinded to each other's results. The pretreatment images were used to identify the primary tumour and nodes. Lymph nodes were then identified on the post-treatment 3DT1W GRE images and drawn on an anatomical map, used as a template to ensure exact lesion-by-lesion matching with nodes harvested at histology. ${ }^{25}$ On T2W-MRI each visible lymph node was scored using a 5-point confidence level score (Table 1). Criteria for malignancy were $>5 \mathrm{~mm}$ in size + spiculated or indistinct border and/or heterogeneous signal pattern. $^{26,27}$

Table 1. Criteria for benign and malignant nodes on T2-weighted MRI

\begin{tabular}{|l||c|}
\hline Confidence Level & \multicolumn{1}{c|}{ Criteria } \\
\hline 0 & size $\leq 5 \mathrm{~mm}+$ regular border/shape + homogeneous signal pattern \\
\hline 1 & size $\leq 5 \mathrm{~mm}+$ irregular border/shape or heterogeneous signal pattern \\
\hline 2 & size $>5$ or irregular border/shape or heterogeneous signal pattern \\
\hline 3 & any size + irregular border/shape + heterogeneous signal pattern \\
\hline 4 & size $>5 \mathrm{~mm}+$ irregular border/shape + heterogeneous signal pattern \\
\hline
\end{tabular}

\section{Visual diffusion analyses}

In the same reading session, the readers evaluated the visibility of lymph nodes on the b1000 images. A lymph node was defined as a round/oval, high signal intensity structure corresponding with a node on the anatomical T1W GRE (Figure 1). The signal of each node was subjectively scored as 'high', 'average' or 'low', with the signal of the primary tumour (on pre-treatment MRI) as the reference for 'high', the signal of the prostate/uterus for 'average' and the signal of the normal rectal wall for 'low'.

\section{ADC analyses}

$A D C$ maps in greyscale were automatically generated at the operating system, using al three b-values. To evaluate interobserver reproducibility, two radiological PhD fellows (DMJL and MM) independently performed ADC measurements of all visible nodes, blinded to each other's results and the radiologists' scores. An oval-shaped region of interest (ROI) was manually drawn for each node on the b1000 images and 
copied to the ADC map. ROI size was chosen to include as much of the nodal parenchyma as possible. ADCs were averaged between the two observers for further diagnostic analyses.

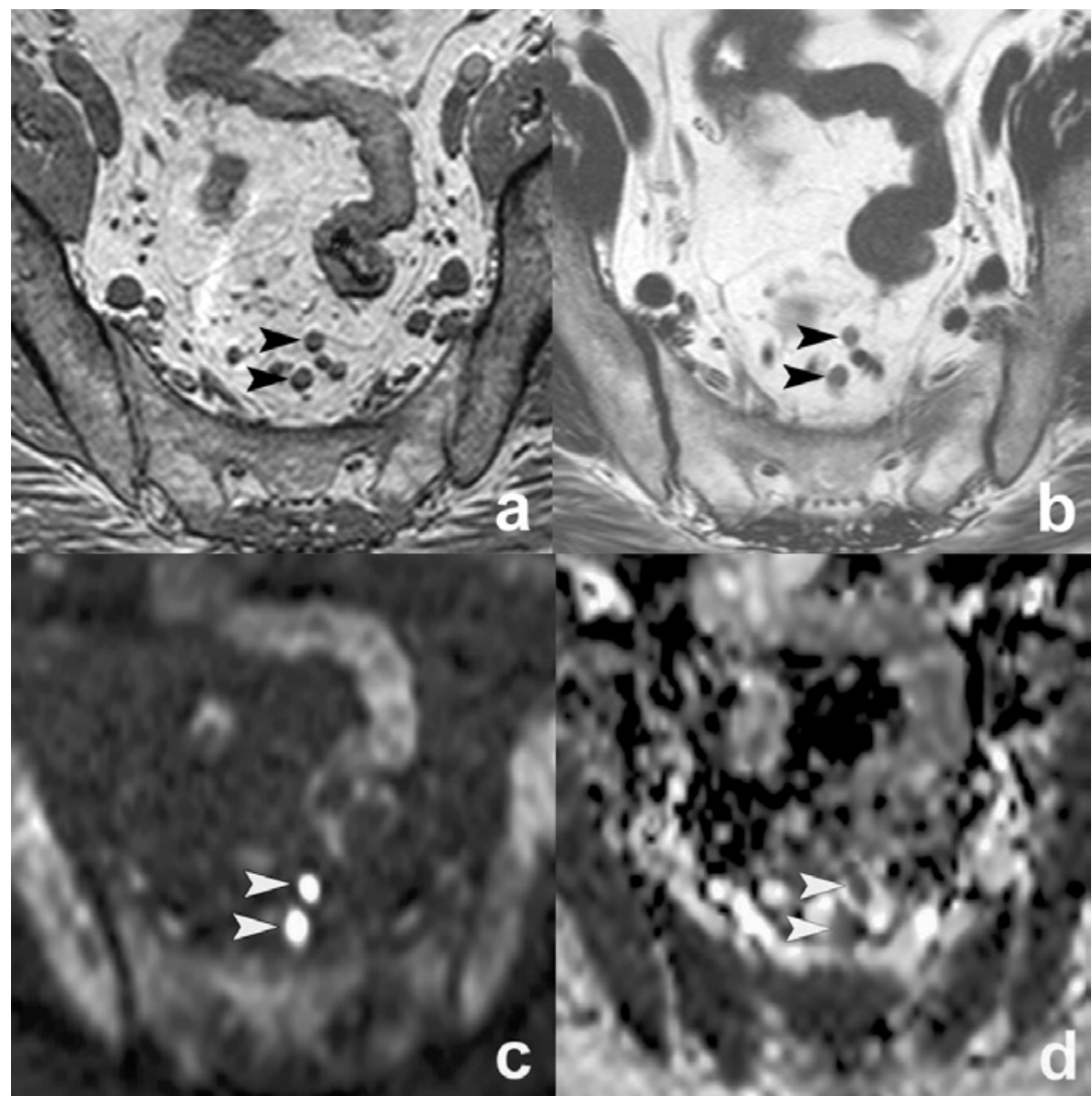

Figure 1. (a) 3DT1W GRE images with 1-mm isotropic voxels were used for accurate depiction of lymph nodes (arrowheads). (b) Nodes were scored as benign or malignant on standard T2W FSE images. (c) Nodes were identified on DWI, where regions of interest were placed and copied to the ADC map. (d) Nodal ADC was calculated from the ADC map including b-values 0,500 and $1000 \mathrm{~s} / \mathrm{mm}^{2}$

\section{Lesion-by-lesion histological evaluation}

All patients underwent a total mesorectal excision. To provide accurate matching with the axial MRI, each specimen was sectioned perpendicular to the rectal lumen, every $5 \mathrm{~mm}$. Each section was searched for lymph nodes by a dedicated pathologist (RGR). Optimal nodal matching was obtained by side by side comparison of the anatomical map with the axially sliced specimen, with special attention for the 
nodal size and the location of the nodes in relation to surrounding structures. ${ }^{25}$. The matching process was performed in consensus by a PhD fellow (DMJL) and the pathologist. Each node was placed in a marked individual tray and processed according to standard methods. For each malignant node the pathologist reported the percentage of the node that contained normal lymphoid tissue, metastatic changes or necrosis, respectively.

\section{Statistical analyses}

Statistical analyses were performed using the Statistical Package for the Social Sciences (SPSS, version 16.0, Inc., Chicago, IL, USA). Weighted kappa values with quadratic weighting were calculated to determine interobserver agreement (IOA) for T2W-MRI. IOA for the nodal ADC measurements was analysed according to the method of Bland and Altman ${ }^{28}$ and by calculating the intraclass correlation coefficient (ICC). A Student's $t$ - test was used to compare ADC (mean of two observers) between benign and malignant nodes. Receiver operator characteristics (ROC) curve analyses were performed to evaluate the diagnostic performance for (a) T2W-MRI, (b) ADC, and (c) the combination of T2W-MRI + ADC. For the latter, a predicted probability derived from a logistic regression analysis (using ADC and the confidence levels from T2W-MRI as independent variables and histologically benign/malignant as the dependent variable) was used as a test variable. Corresponding areas under the ROC curve (AUC), sensitivity, specificity, positive and negative predictive values and overall accuracy were calculated. For T2W-MRI the confidence levels were dichotomised with the cut-off set between 2 (possibly malignant) and 3 (probably malignant). For the ADC and combined T2W + ADC, the cut-offs were determined according to the point nearest to the upper left corner in the ROC curves. Each node that was not identified on T2W-MRI and/or DWI was assigned a negative score. Differences in diagnostic performance were analysed by comparing the ROC curves according to the method described by DeLong et al. ${ }^{29} P$ values $<0.05$ were considered statistically significant.

\section{Results}

\section{Histopathology}

At histology 19 patients had a ypN0, 7 a ypN1 and 4 a ypN2 status. A total number of 321 nodes was harvested (median 12 nodes per patient; range 1-24; $281 \mathrm{~N}-, 40$ $\mathrm{N}+$ ). A node-by-node MR-histological analysis of 222 nodes could be performed (median size $3 \mathrm{~mm}$, range 2-16 mm), of which 188 were benign and 34 were 
malignant. The other 99 harvested nodes were excluded because they could not be matched with MRI.

\section{Interobserver agreement}

IOA for the confidence level scores on T2W-MRI was good ( $\mathrm{k} 0.73$ ). IOA for the visual (signal intensity) evaluation on DWI was moderate ( $к .43$ ). The Bland-Altman plot for the ADC measurements is displayed in Figure 2. The mean bias in ADC between the two readers was $0.064 * 10^{-3} \mathrm{~mm}^{2} / \mathrm{s}$ and limits of agreement were $\pm 0.442 * 10^{-3} \mathrm{~mm}^{2} / \mathrm{s}$. The ICC was 0.77 .

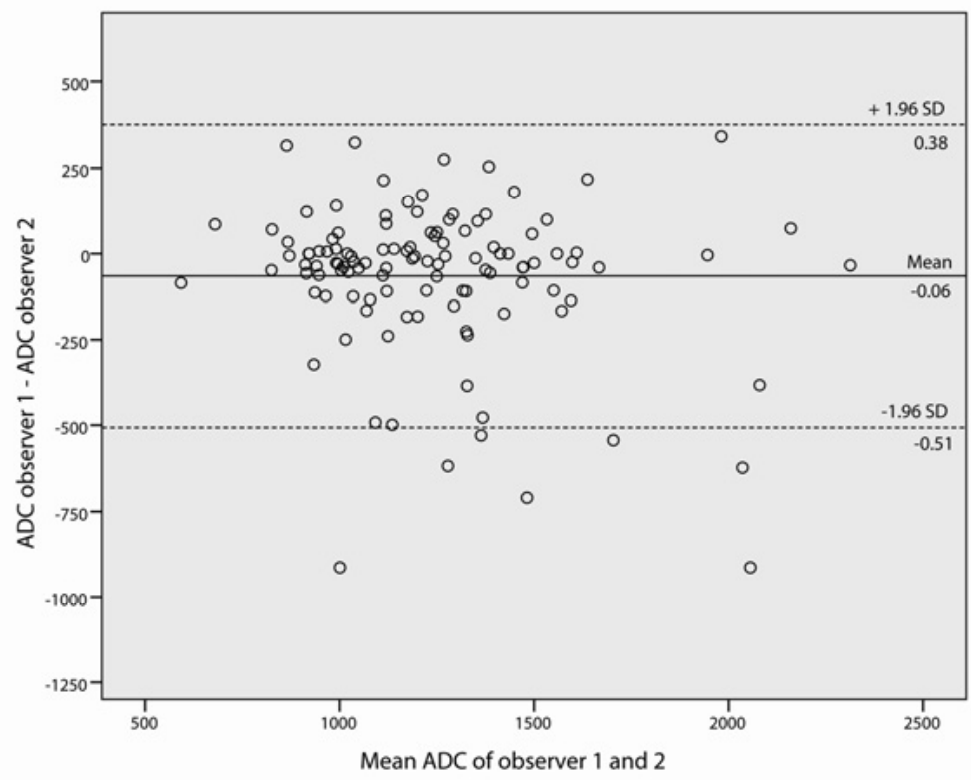

Figure 2. Interobserver reproducibility for nodal ADC measurements. Bland-Altman plot of the mean $A D C$ of the two observers ( $x$-axis) against the difference in ADC between the two observers (y-axis). The continuous line represents the mean absolute difference (bias) in ADC between the two observers; the dashed lines represent the $95 \%$ confidence intervals of the mean difference (limits of agreement)

\section{Diagnostic performance of T2W-MRI}

On T2W-MRI, 101/222 (45\%) nodes were identified (median size $4 \mathrm{~mm}$; range 2-16 $\mathrm{mm} ; 27 \mathrm{~N}+, 74 \mathrm{~N}-)$. The AUC for detection of metastatic nodes was 0.88 for reader 1 and 0.95 for reader 2 . Sensitivity was $65 \%$, specificity $93 \%$, PPV $61 \%$ and NPV $94 \%$ for reader 1 . These values were $71 \%, 91 \%, 60 \%$ and $95 \%$ for reader 2 . 


\section{Visual evaluation of DWI}

On the b1000 images, 157/222 (71\%) nodes were identified (median size $3 \mathrm{~mm}$; range $2-16 \mathrm{~mm} ; 37 \mathrm{~N}+, 180 \mathrm{~N}-$ ). Signal intensities did not differ between the benign and metastatic nodes (Figure 3 ) and rendered an AUC of $0.64(95 \% \mathrm{Cl} 0.53-0.75)$ for reader 1 and 0.52 (95\% $\mathrm{Cl} 0.40-0.64)$ for reader 2 .

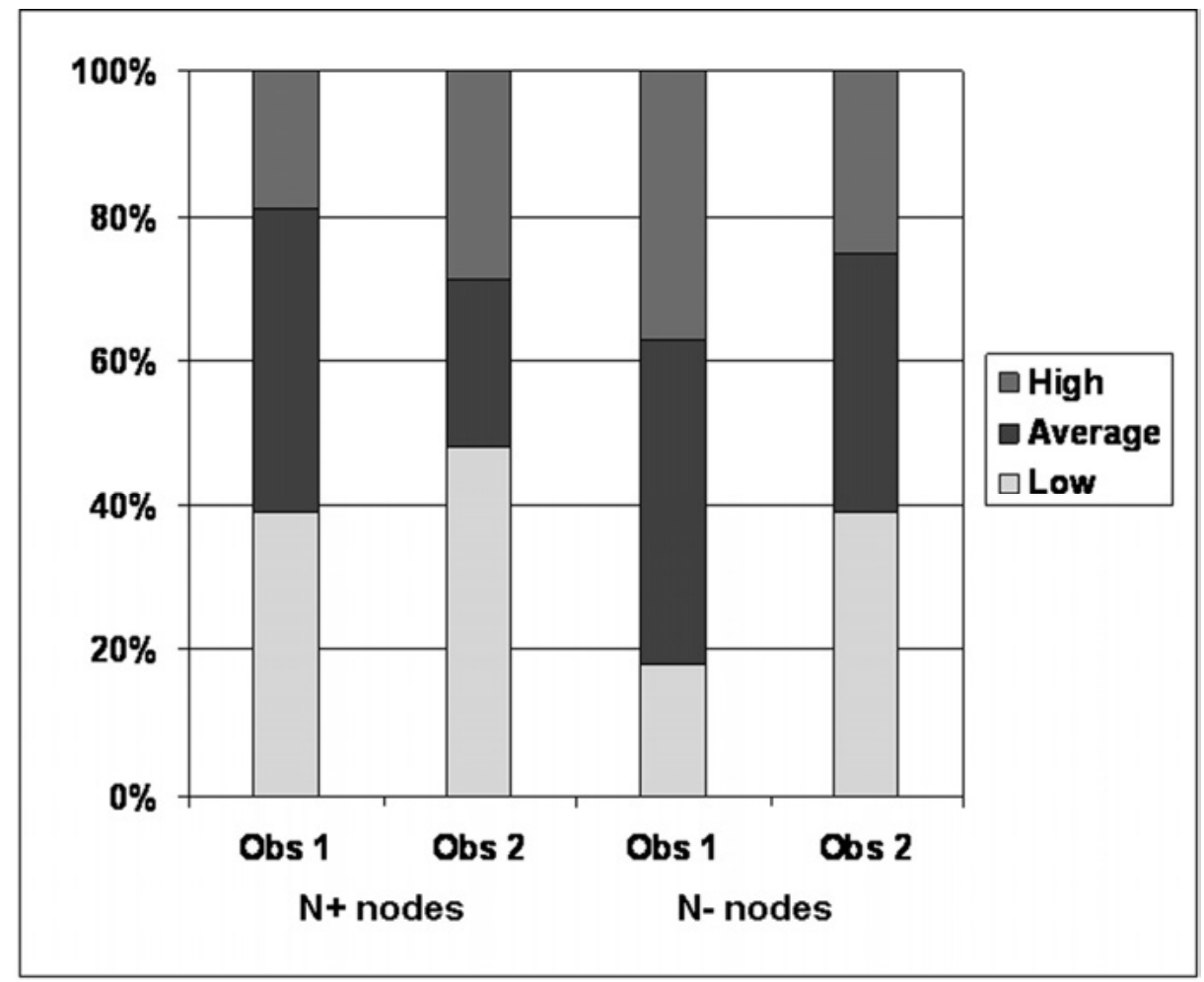

Figure 3. Distribution of subjectively scored signal intensities on DWI for benign and malignant nodes. The bars represent the proportion (\%) of 'low', 'average' and 'high' signal intensities for the two readers

\section{Diagnostic performance of ADC}

Of the 157 nodes identified on DWI, ADC measurements could be obtained in 115 nodes. The remaining 42 nodes were excluded because the nodes were too small to place an ROI or the standard errors of the ADC measurements were too large, because of local image distortions. Mean ADC was $1.19 \pm 0.27 * 10^{-3} \mathrm{~mm}^{2} / \mathrm{s}$ for the benign and $1.43 \pm 0.38 * 10^{-3} \mathrm{~mm}^{2} / \mathrm{s}$ for the malignant nodes $(p<0.001)$. AUC for 
detection of metastatic nodes was 0.66 . The optimal ADC threshold was $1.25 * 10^{-3}$ $\mathrm{mm}^{2} / \mathrm{s}$, resulting in a sensitivity of $53 \%$, specificity $82 \%$, PPV $35 \%$ and NPV $91 \%$.

\section{Diagnostic performance of T2W-MRI + ADC}

For 69 nodes, ADCs and T2W confidence level scores could be combined. For the other nodes, ADC was not available and/or the nodes were not visualised on T2WMRI. The predicted probability for the combined assessment of T2W-MRI + ADC derived from the logistic regression rendered an AUC of 0.91 for reader 1 and 0.96 for reader 2 . The optimal predicted probability was 0.51 for reader 1 and 0.69 for reader 2 , which resulted in a sensitivity of $56 \%$, specificity $98 \%$, PPV $83 \%$ and NPV $92 \%$ for reader 1 . These values were 56\%, 99\%, 95\% and 93\% for reader 2 .

\begin{tabular}{|c|c|c|c|c|c|}
\hline & \multicolumn{2}{|c|}{$\mathrm{T} 2 \mathrm{~W}$} & \multirow[t]{2}{*}{ Mean ADC } & \multicolumn{2}{|c|}{$T 2 W+A D C$} \\
\hline & R1 & R2 & & R1 & R2 \\
\hline Sensitivity & $65(22 / 34)$ & $71(24 / 34)$ & $53(18 / 34)$ & $56(19 / 34)$ & $56(19 / 34)$ \\
\hline $95 \% \mathrm{Cl}$ & $51-76$ & $57-82$ & $38-67$ & $44-63$ & $46-58$ \\
\hline Specificity & $93(174 / 188)$ & $91(172 / 188)$ & $82(154 / 188)$ & $98(184 / 188)$ & $99(187 / 188)$ \\
\hline $95 \% \mathrm{Cl}$ & $90-95$ & $89-94$ & $79-84$ & $96-99$ & $98-100$ \\
\hline PPV & $61(22 / 36)$ & $60(24 / 40)$ & $35(18 / 52)$ & $83(19 / 23)$ & $95(19 / 20)$ \\
\hline $95 \% \mathrm{Cl}$ & $48-72$ & $48-69$ & $25-44$ & $66-93$ & $79-99$ \\
\hline NPV & $94(174 / 186)$ & $95(172 / 182)$ & $91(154 / 170)$ & 92 (184/199) & $93(187 / 202)$ \\
\hline $95 \% \mathrm{Cl}$ & $91-96$ & $92-97$ & $88-93$ & $91-94$ & $91-93$ \\
\hline Accuracy & $88(196 / 222)$ & $88(196 / 222)$ & $77(172 / 222)$ & $91(203 / 222)$ & $93(206 / 222)$ \\
\hline $95 \% \mathrm{Cl}$ & $84-92$ & $84-92$ & $73-82$ & $88-94$ & $90-94$ \\
\hline AUC & 0.88 & 0.95 & 0.66 & 0.91 & 0.96 \\
\hline $95 \% \mathrm{Cl}$ & $0.78-0.97$ & $0.90-1.0$ & $0.51-0.81$ & $0.82-1.0$ & $0.92-1.0$ \\
\hline
\end{tabular}

Numbers are percentages. Absolute numbers are given in parentheses. PPV = positive predictive value, $\mathrm{NPV}=$ negative predictive value, $\mathrm{AUC}=$ area under the curve, $95 \% \mathrm{Cl}=95 \%$ Confidence Interval.

The diagnostic predictive values for the three reading methods are displayed in Table 2. The ROC curves are displayed in Figure 4. The diagnostic performance when using ADC only was significantly lower than for T2W-MRI $(P=$ 0.02 and $P=0.0003$ for readers 1 and 2 , respectively) and T2W-MRI + ADC combined $(P=0.001$ and $P<0.0001)$. There was no significant difference in 
diagnostic performance between T2W-MRI and the combination of T2W + ADC $(P=$ 0.17 and $P=0.26)$.

\section{Histological features of the malignant nodes}

In $22 \%$ of the malignant nodes, $>50 \%$ of the node consisted of necrosis. In $17 \%$ of the nodes, $10-30 \%$ of the node was necrotic (Figure $5 \mathrm{~b}$ ). In the other $61 \%$ of nodes there was no or only a little $(<5 \%)$ necrosis. Of these nodes, a normal lymphoid architecture was preserved in a large proportion ( $>20 \%$ ) of the node in $45 \%$ of the cases (Figure $5 \mathrm{a}$ ). These nodes with a relatively preserved lymphoid architecture showed low ADC values $\left(1.16 \pm 0.14 * 10^{-3} \mathrm{~mm}^{2} / \mathrm{s}\right)$ that were comparable to the benign nodes $\left(1.19 \pm 0.27 * 10^{-3} \mathrm{~mm}^{2} / \mathrm{s}, P=0.49\right)$ but were lower compared with the nodes that were fully metastatic or necrotic $\left(1.40 \pm 0.28 * 10^{-3} \mathrm{~mm}^{2} / \mathrm{s}\right)$, although this difference did not reach statistical significance $(P=0.08)$.

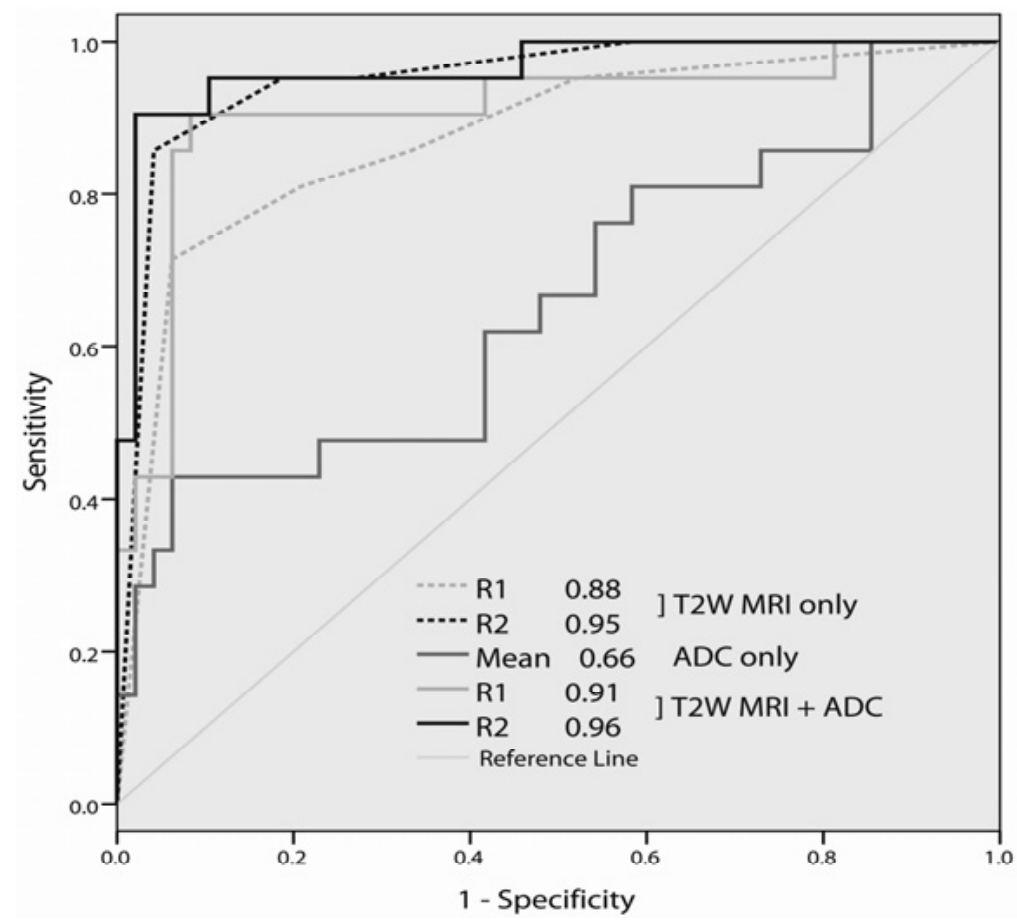

Figure 4. ROC curves used to evaluate the diagnostic performance for the detection of metastatic nodes for [a] T2-weighted (T2W) MRI only, [b] ADC measurements only and [c] for the combined assessment of T2W-MRI + ADC. There was no significant difference in performance between the use of T2W-MRI only, and the combination of T2W-MRI + ADC ( $p=0.17$ for reader 1 and 0.26 for reader 2 ). The use of $A D C$ measurements only resulted in significantly worse performance than for T2W-MRI only or the combination of T2W-MRI + ADC 


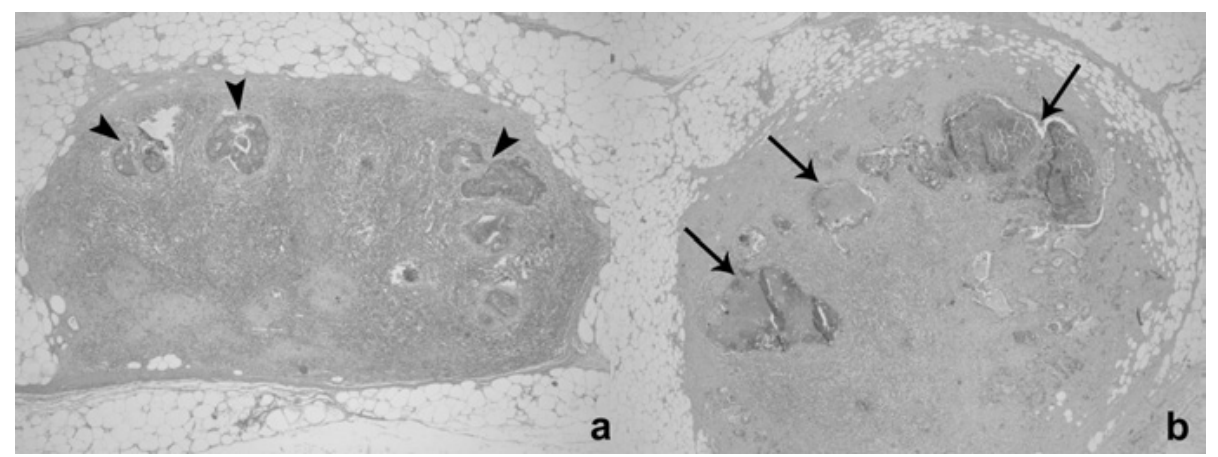

Figure 5. Histological evaluation of 2 metastatic lymph nodes (haematoxylin and eosin staining; original magnification 50x). (a) Lymph node with a relatively preserved lymphoid architecture, where only small nests of metastatic adenocarcinoma can be visualised (arrowheads). This node still resembled a healthy lymph node and showed relatively low ADC. (b) Example of a node with large areas of necrosis (arrows). Because of the decreased cellular density in these necrotic areas, this node showed a relatively high ADC

\section{Discussion}

The goal of this study was to evaluate the diagnostic performance of DWI in addition to standard T2W-MRI for the evaluation of rectal cancer lymph nodes after neoadjuvant chemoradiation treatment. Our results indicate that visual DWI evaluation improves the number of lymph nodes detected (from $45 \%$ to $71 \%$ ) but that it is not useful for discrimination between benign and metastatic nodes. The mean ADCs of lymph nodes are discriminative with significantly higher values for the malignant nodes. However, we found an overlap in ADC between the benign and malignant nodes and insufficient accuracy when using $A D C$ only for the detection of nodal metastases. ADC combined with standard T2W-MRI improved the diagnostic performance, however without reaching a significant improvement compared with T2W-MRI only.

Our results thus suggest that after chemoradiation, T2W-MRI on its own is already sufficient for nodal evaluation, reaching an AUC of 0.88-0.95. Such good results have also been reported by previous authors, with high NPVs ranging between 81 and 100\%, indicating that a restaging MRI after CRT can accurately identify the ypNO patients. ${ }^{10,11}$ This is remarkable, as it is known from two recent meta-analyses that MRI generally performs poorly for nodal staging. ${ }^{8,9}$ It has to be noted, however, that these meta-analyses only included studies that focussed on primary nodal staging. Apparently, morphological criteria (size, shape and border) work better in a restaging setting. Many small $(2-5 \mathrm{~mm})$ nodes disappear after CRT, while up to $50 \%$ of these nodes initially contained metastases. ${ }^{30,31}$ of the small nodes that remain visible after CRT, up to $90 \%$ are sterilised. ${ }^{32}$ Nodes that remain 
large are thus more likely to be malignant. Overall this facilitates a more reliable assessment of nodes based on morphological criteria only on a restaging MRI.

We found a significant difference in ADC between benign and malignant nodes. Nevertheless, there was a considerable overlap in values, making it difficult to identify a clinically reliable threshold for malignancy. With the optimal threshold of $1.25 * 10^{-3} \mathrm{~mm}^{2} / \mathrm{s}$, a high NPV of $91 \%$ could only be obtained at the cost of a low PPV of $35 \%$. This is in concordance with the previous results of Kim et al who measured nodal ADCs in 125 cervical cancer patients and achieved a NPV of $99 \%$ on a patient basis, but a PPV of only $38 \%$, leading to overstaging in a huge proportion of patients. ${ }^{19}$ Of interest was our finding that, although it did not improve the overall performance, the addition of ADC to standard T2W-MRI did improve the PPV from $60-61 \%$ to $83-95 \%$, thus reducing the overstaging errors.

Contrary to most nodal DWI reports, we found significantly higher ADCs for the metastatic nodes. This discrepancy may be attributed to the fact that we included patients who all had undergone preoperative chemoradiation. It is known that irradiated malignant nodes often become (partly) necrotic. Due to a loss of cell structure, these necrotic areas are associated with increased diffusivity, resulting in higher ADCs. At histology, large areas of necrosis were present in over one third of the metastatic nodes (Figure 5b), which may explain the higher ADCs found for the malignant nodes in our study. Previous studies in head/neck and oesophageal cancer also reported higher ADCs in metastatic nodes and found necrotic changes to be the major reason. ${ }^{16,17,33}$ Interestingly, in studies that reported lower ADC values, none of the patients had been irradiated. ${ }^{14,15,19-21,23}$

Interobserver agreement for ADC measurements was good (ICC 0.77). However, the limits of agreement from the Bland-Altman plot $\left( \pm 0.442 * 10^{-3} \mathrm{~mm}^{2} / \mathrm{s}\right)$ exceeded the mean absolute difference of $0.240 * 10^{-3} \mathrm{~mm}^{2} / \mathrm{s}$ in ADC between the benign and malignant nodes, thus limiting the clinical utility of ADC. This was also observed in a previous study by Kwee et al. ${ }^{34}$. Although ADC measurements provide objective, quantifiable information, the manual placement of ROIs remains a subjective action and will limit reproducibility.

The ADC thresholds in our study were only tested retrospectively, whereas ideally these thresholds should be tested prospectively. At the same time we realise that measuring nodal ADCs requires a considerable time investment from a radiologist in a busy clinical practice. Furthermore, ADC measurements are influenced by MR equipment, imaging parameters and methods of analyses. It will therefore be difficult to obtain uniform thresholds that can be broadly implemented into clinics. Further research should therefore focus on standardising imaging and analyses protocols. 
The main gain from the addition of DWI was the improved number of detected nodes compared with T2W-MRI. On DWI, high signal intensity nodes were more easily detected against the suppressed background signal of the surrounding tissues. DWI can thus be used to immediately focus a radiologist's eye on the presence of nodes and reduce the time needed for the evaluation of nodal status.

\section{Clinical impact}

Accurate nodal restaging after chemoradiation may have a substantial impact on therapeutic decision-making. While at present all patients with locally advanced rectal cancer undergo preoperative chemoradiation followed by a total mesorectal excision, minimally invasive treatments could be a safe alternative when patients with a node-negative status and a good response of the primary tumour can be accurately selected. Although these treatment alternatives are still under debate, the gradual shift within the near future will be towards organ saving surgery for the good responders. When we can provide a tool for better selection of these patients, in particular for the selection of those with truly sterilised nodes, patients with a small tumour remnant (ypT1-2N0) may be safely stratified for a local excision, while patients with a complete response (ypTONO) could go for a wait-and-see policy. ${ }^{6,7}$

In conclusion, this study shows that after chemoradiation ADC measurements may have the potential for nodal characterisation in rectal cancer, but DWI as a stand alone technique is not reliable. The main gain from the addition of DWI is an increase in the number of detected nodes and an improved PPV for identification of metastatic nodes. However, it does not improve overall diagnostic performance and after CRT, T2W-MRI on its own is already sufficiently accurate. 


\section{References}

1. Gosens MJ, van Krieken JH, Marijnen CA, et al. Improvement of staging by combining tumor and treatment parameters: the value for prognostication in rectal cancer. Clin Gastroenterol Hepatol 2007;5(8):997-1003.

2. Valentini V, Coco C, Minsky BD, et al. Randomized, multicenter, phase llb study of preoperative chemoradiotherapy in T3 mid-distal rectal cancer: raltitrexed + oxaliplatin + radiotherapy versus cisplatin + 5-fluorouracil + radiotherapy. Int J Radiat Oncol Biol Phys 2008;70(2):403-412.

3. Janjan NA, Khoo VS, Abbruzzese J, et al. Tumor downstaging and sphincter preservation with preoperative chemoradiation in locally advanced rectal cancer: the M. D. Anderson Cancer Center experience. Int J Radiat Oncol Biol Phys 1999;44(5):1027-1038.

4. Rodel C, Martus P, Papadoupolos T, et al. Prognostic significance of tumor regression after preoperative chemoradiotherapy for rectal cancer. J Clin Oncol 2005;23(34):8688-8696.

5. Vecchio FM, Valentini V, Minsky BD, et al. The relationship of pathologic tumor regression grade (TRG) and outcomes after preoperative therapy in rectal cancer. Int J Radiat Oncol Biol Phys $2005 ; 62(3): 752-760$.

6. Lezoche G, Baldarelli M, Guerrieri M, Paganini AM, De Sanctis A, Bartolacci S, Lezoche E. A prospective randomized study with a 5-year minimum follow-up evaluation of transanal endoscopic microsurgery versus laparoscopic total mesorectal excision after neoadjuvant therapy. Surg Endosc 2008;22(2):352-358.

7. Habr-Gama A, Perez RO, Proscurshim I, Campos FG, Nadalin W, Kiss D, Gama-Rodrigues J. Patterns of failure and survival for nonoperative treatment of stage $\mathrm{c} 0$ distal rectal cancer following neoadjuvant chemoradiation therapy. J Gastrointest Surg 2006;10(10):1319-1328; discussion 28-29.

8. Bipat S, Glas AS, Slors FJ, Zwinderman AH, Bossuyt PM, Stoker J. Rectal cancer: local staging and assessment of lymph node involvement with endoluminal US, CT, and MR imaging--a meta-analysis. Radiology 2004;232(3):773-783.

9. Lahaye MJ, Engelen SM, Nelemans PJ, Beets GL, van de Velde CJ, van Engelshoven JM, Beets-Tan RG. Imaging for predicting the risk factors--the circumferential resection margin and nodal disease--of local recurrence in rectal cancer: a meta-analysis. Semin Ultrasound CT MR 2005;26(4):259-268.

10. Lahaye MJ, Beets GL, Engelen SM, et al. Locally advanced rectal cancer: MR imaging for restaging after neoadjuvant radiation therapy with concomitant chemotherapy. Part II. What are the criteria to predict involved lymph nodes? Radiology 2009;252(1):81-91.

11. Suppiah A, Hunter IA, Cowley J, Garimella V, Cast J, Hartley JE, Monson JR. Magnetic resonance imaging accuracy in assessing tumour down-staging following chemoradiation in rectal cancer. Colorectal Dis 2009;11(3):249-253.

12. Kwee TC, Takahara T, Ochiai R, Nievelstein RA, Luijten PR. Diffusion-weighted whole-body imaging with background body signal suppression (DWIBS): features and potential applications in oncology. Eur Radiol. 2008;18(9):1937-1952.

13. Nakai G, Matsuki M, Inada $Y$, Tatsugami F, Tanikake M, Narabayashi I, Yamada T. Detection and evaluation of pelvic lymph nodes in patients with gynecologic malignancies using body diffusionweighted magnetic resonance imaging. J Comput Assist Tomogr 2008;32(5):764-768.

14. de Bondt RB, Hoeberigs MC, Nelemans PJ, Deserno WM, Peutz-Kootstra C, Kremer B, Beets-Tan RG. Diagnostic accuracy and additional value of diffusion-weighted imaging for discrimination of malignant cervical lymph nodes in head and neck squamous cell carcinoma. Neuroradiology 2009;51(3):183-192.

15. Vandecaveye V, De Keyzer F, Vander Poorten V, Dirix P, Verbeken E, Nuyts S, Hermans R. Head and neck squamous cell carcinoma: value of diffusion-weighted MR imaging for nodal staging. Radiology 2009;251(1):134-146.

16. Sumi M, Sakihama N, Sumi T, et al. Discrimination of metastatic cervical lymph nodes with diffusionweighted MR imaging in patients with head and neck cancer. AJNR Am J Neuroradiol 2003;24(8):1627-1634. 
17. Sumi M, Van Cauteren M, Nakamura T. MR microimaging of benign and malignant nodes in the neck. AJR Am J Roentgenol 2006;186(3):749-757.

18. Lin G, Ho KC, Wang JJ, et al. Detection of lymph node metastasis in cervical and uterine cancers by diffusion-weighted magnetic resonance imaging at 3T. J Magn Reson Imaging 2008;28(1):128-135.

19. Kim JK, Kim KA, Park BW, Kim N, Cho KS. Feasibility of diffusion-weighted imaging in the differentiation of metastatic from nonmetastatic lymph nodes: early experience. J Magn Reson Imaging 2008;28(3):714-719.

20. Park SO, Kim JK, Kim KA, et al. Relative apparent diffusion coefficient: determination of reference site and validation of benefit for detecting metastatic lymph nodes in uterine cervical cancer. J Magn Reson Imaging 2009;29(2):383-390.

21. Holzapfel K, Duetsch S, Fauser C, Eiber M, Rummeny EJ, Gaa J. Value of diffusion-weighted MR imaging in the differentiation between benign and malignant cervical lymph nodes. Eur J Radiol 2009;72(3):381-387.

22. King AD, Ahuja AT, Yeung DK, Fong DK, Lee YY, Lei KI, Tse GM. Malignant cervical lymphadenopathy: diagnostic accuracy of diffusion-weighted MR imaging. Radiology 2007;245(3):806-813.

23. Abdel Razek AA, Soliman NY, Elkhamary S, Alsharaway MK, Tawfik A. Role of diffusion-weighted MR imaging in cervical lymphadenopathy. Eur Radiol 2006;16(7):1468-1477.

24. Takahara T, Imai Y, Yamashita T, Yasuda S, Nasu S, Van Cauteren M. Diffusion weighted whole body imaging with background body signal suppression (DWIBS): technical improvement using free breathing, STIR and high resolution 3D display. Radiat Med 2004;22(4):275-282

25. Lahaye MJ, Engelen SME, Kessels $A G H$, et al. USPIO-enhanced MR Imaging for nodal staging in patients with primary rectal cancer: Predictive criteria. Radiology 2008;246(3):804-811

26. Brown G, Richards CJ, Bourne MW, Newcombe RG, Radcliffe AG, Dallimore NS, Williams GT. Morphologic predictors of lymph node status in rectal cancer with use of high-spatial-resolution MR imaging with histopathologic comparison. Radiology 2003;227(2):371-377.

27. Kim JH, Beets GL, Kim MJ, Kessels AG, Beets-Tan RG. High-resolution MR imaging for nodal staging in rectal cancer: are there any criteria in addition to the size? Eur J Radiol 2004;52(1):78-83.

28. Bland JM, Altman DG (1986) Statistical methods for assessing agreement between two methods of clinical measurement. Lancet. 1(8476):307-310.

29. DeLong ER, DeLong DM, Clarke-Pearson DL. Comparing the areas under two or more correlated receiver operating characteristic curves: a nonparametric approach. Biometrics 1988;44(3):837-845.

30. Dworak O. Number and size of lymph nodes and node metastases in rectal carcinomas. Surg Endosc 1989;3(2):96-99.

31. Wang C, Zhou Z, Wang Z, et al. Patterns of neoplastic foci and lymph node micrometastasis within the mesorectum. Langenbecks Arch Surg 2005;390(4):312-318.

32. Koh DM, Chau I, Tait D, Wotherspoon A, Cunningham D, Brown G. Evaluating mesorectal lymph nodes in rectal cancer before and after neoadjuvant chemoradiation using thin-section T2-weighted magnetic resonance imaging. Int J Radiat Oncol Biol Phys 2008;71(2):456-461.

33. Sakurada A, Takahara T, Kwee TC, et al. Diagnostic performance of diffusion-weighted magnetic resonance imaging in esophageal cancer. Eur Radiol 2009;19(6):1461-1469.

34. Kwee TC, Takahara T, Luijten PR, Nievelstein RA. ADC measurements of lymph nodes: Inter- and intra-observer reproducibility study and an overview of the literature. Eur J Radiol 2010;75:215-220. 


\section{ACCURACY OF GADOFOSVESET-ENHANCED MRI FOR NODAL STAGING AND RESTAGING IN RECTAL CANCER}

\section{CHAPTER}

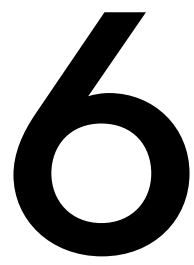

DMJ Lambregts

GL Beets M Maas AGH Kessels FCH Bakers VC Cappendijk SME Engelen MJ Lahaye $A P$ de Bruïne $G$ Lammering T Leiner JL Verwoerd JE Wildberger RGH Beets-Tan

Published in: Annals of Surgery 2011; 253(3):539-545 


\section{Abstract}

Aim

Accurate preoperative assessment of nodal disease in rectal cancer impacts treatment management. Staging with modern imaging techniques (CT, MRI and endorectal ultrasound) is insufficiently accurate for clinical decision making. This study aims to prospectively assess the accuracy of MRI using a novel lymph node MR-contrast, gadofosveset, for nodal staging and restaging in rectal cancer using a per node comparison with histology as the reference standard.

\section{Materials \& Methods}

68 patients underwent gadofosveset-enhanced MRI at 1.5T. Twenty-six patients (primary staging group I) were treated with total mesorectal excision (with or without preoperative $5 \times 5 \mathrm{~Gy}$ ) and 42 (restaging group II) underwent a long course of chemoradiation followed by a restaging MRI and resection. Nodes were scored as benign or malignant by two radiologists (experienced and junior reader): first on standard MRI, then on gadofosveset-enhanced MRI. For group I the primary staging MRI was compared with histology. In group II the second, restaging MRI was compared with histology.

\section{Results}

For the experienced reader, sensitivity, specificity, and area under the ROC-curve (AUC) improved from $76 \%, 82 \%$ and 0.84 on standard $\mathrm{MRI}$ to $80 \%, 97 \%$ and 0.96 on gadofosveset-MRI $(P<0.001)$. For the junior reader results improved from $69 \%$, $85 \%$ and 0.85 on standard $\mathrm{MRI}$ to $70 \%, 95 \%$ and 0.93 on gadofosveset-MRI $(P=0.03)$. Interobserver agreement was good on both standard MRI $(\kappa 0.73)$ and gadofosveset-MRI ( $\kappa$ 0.71).

\section{Conclusions}

This study shows high reproducibility and significantly improved accuracy compared to standard MRI for gadofosveset-enhanced MRI for nodal staging and restaging in rectal cancer. 


\section{Introduction}

In the last decades there has been a shift from postoperative to preoperative (chemo)radiotherapy for rectal cancer patients at risk for local recurrence. ${ }^{1}$ Whereas previously risk assessment was primarily based on histology of the resection specimen, preoperative imaging now has to provide information on the most important risk factors: T-stage, $\mathrm{N}$-stage and the circumferential resection margin (CRM). ${ }^{2-4}$ Endoluminal ultrasound (EUS) is traditionally used for T-staging, while MRI has recently been promoted mainly for the assessment of the primary tumour and $\mathrm{CRM}^{5}{ }^{5-6}$ With both techniques, however, the detection of metastatic nodes remains difficult. Studies that analysed pooled data from many centers showed that EUS, CT, and MRI all lack sufficient accuracy for identifying nodal metastases, with sensitivities and specificities ranging between $55-78 \%{ }^{7,8}$ Despite advances in high resolution imaging, understaging of the nodal status occurs in up to $16 \%$ on MRI and $25 \%$ on EUS. ${ }^{9}$ This is because distinction between benign and malignant nodes is mainly based on size and shape. These criteria are not reliable in rectal cancer, in particular for nodes smaller than $5 \mathrm{~mm}$, which are involved in up to $50 \%$ of the cases. ${ }^{10-12}$

The only imaging method that showed promising results for nodal staging in rectal cancer was Ultrasmall Superparamagnetic Particles of Iron Oxide (USPIO) enhanced MRI. ${ }^{13-15}$ However, so far USPIO has not been FDA approved. An alternative MR contrast agent that - in preclinical and clinical studies - has shown promise for lymph node characterisation is gadofosveset trisodium. ${ }^{16-18}$ A non-invasive imaging method that could accurately assess the nodal stage would enable further tailoring of treatment in rectal cancer. When the truly node negative patients can be selected at primary staging, these patients may undergo direct surgery without the need for preoperative CRT. ${ }^{3}$ Small, node negative tumours (T12) may even be treated with a local excision. ${ }^{19}$ For patients treated with neoadjuvant CRT, there is now a trend towards minimally invasive treatments and organ saving surgery when the tumour and nodes have responded well to therapy. Although these options are still considered investigational and controversial, patients with a small tumour remnant could undergo a local excision, while patients with a complete response of the tumour and nodes may even go for a 'wait-andsee' policy without further surgery, but only when the truly node negative patients after CRT are accurately selected. ${ }^{20-22}$

Therefore, the aim of this prospective study was to evaluate the accuracy of gadofosveset-enhanced MRI for primary nodal staging and restaging after CRT in rectal cancer. In order to validate the use of this novel contrast for characterisation 
of individual lymph nodes, results were analysed on a lesion by lesion basis, using a per node comparison with histology as the reference standard.

\section{Materials \& Methods}

The study was approved by the local institutional review board. Written informed consent was obtained from all patients prior to inclusion.

\section{Patients}

Between November 2006 and November 2009, 118 consecutive rectal cancer patients were considered for inclusion. Inclusion criteria consisted of a) biopsy proven rectal cancer with an inferior tumour margin $<15 \mathrm{~cm}$ from the anal verge and b) eligibility to provide informed consent. Exclusion criteria were (a) pregnancy, (b) non-resectable disease, (c) contra-indications for $\mathrm{MRI}^{23}$, and (d) impaired kidney function (glomerular filtration rate $<30 \mathrm{ml} / \mathrm{min}$ ). Of these 118 patients, 50 were excluded for the following reasons: 18 patients declined participation, two died during treatment, 29 refused surgery or had non-resectable disease and one patient had impaired kidney function. This left a total of 68 patients (48 male, 20 female; median age 70 years, range $34-88$ ) that were included in the study.

All patients underwent a standard staging MRI and an additional gadofosveset-enhanced imaging sequence. Based on assessment of the risk factors for local recurrence on standard MRI ( tumour location, T-stage, CRM and nodal status), patients were categorised into different treatment groups: the low risk patients $(n=6)$ underwent immediate Total Mesorectal Excision (TME), the intermediate risk patients $(n=20)$ a short course of $5 \times 5$ Gy radiotherapy immediately followed by TME and the high risk patients $(n=42)$ a long course of chemoradiation (CRT) followed by a second, restaging MRI 6-8 weeks after completion of CRT plus subsequent surgery. The CRT consisted of $28 \times 1.8 \mathrm{~Gy}$ combined with $2 \times 825 \mathrm{mg} / \mathrm{m}^{2} / \mathrm{d}$ Capecitabine.

For evaluation purposes two subgroups of patients were considered. Group I (the "primary staging group") consisted of the 26 patients who underwent immediate TME, or had TME within one week after 5x5 Gy. These patients were grouped together because $5 \times 5 \mathrm{~Gy}+$ immediate TME is reported not to influence the nodal stage in rectal cancer. ${ }^{24}$ For group I the primary staging MRI was compared with histology. Group II (the "restaging post-CRT group") consisted of the 42 patients who underwent a long course of CRT. In group II, the second, restaging MRI was compared with histology. 


\section{MR imaging}

Patients did not receive bowel preparation or spasmolytics. Imaging was performed at 1.5T (Intera; Philips Medical Systems, Best, The Netherlands) using a phased array body coil. The standard MR staging protocol consisted of 2D T2-weighted fast spin echo sequences (TR/TE 3427/150 msec, 90 flip angle, 25 echotrain length, 6 NSA, $0.78 \times 1.14 \times 5.00 \mathrm{~mm}$ voxel size, $5.08 \mathrm{~min}$ acquisition time) in three planes (sagittal, axial and coronal). For study purposes, a gadofosveset-enhanced 3D T1-weighted gradient echo sequence (TR/TE 9.8/4.6 msec, $15^{\circ}$ flip angle, 1 NSA, $1.15 \times 1.15 \times 1.00$ $\mathrm{mm}$ voxel size, $6.30 \mathrm{~min}$ acquisition time) was added to the standard protocol.

\section{Gadofosveset contrast agent}

Gadofosveset is a blood pool MR contrast agent that binds to human albumin and is originally marketed for vascular MR imaging. During the study period gadofosveset was marketed in Europe as Vasovist (Bayer Schering Pharma, Berlin, Germany ${ }^{16}$ ) Recently, gadofosveset was launched in the US under the trade name Ablavar ${ }^{\mathrm{TM}}$, Lantheus Medical Imaging, Billerica, MA,USA). Gadofosveset was administered by manual bolus injection through an intravenous line. The dose was $0.12 \mathrm{ml} / \mathrm{kg}$ bodyweight. The gadofosveset-enhanced images were obtained 17 minutes after contrast administration. ${ }^{18}$

\section{Image evaluation}

Each lymph node visible on MRI was prospectively assessed by a gastrointestinal (GI) radiologist with thirteen years experience in pelvic MRI (reader $1, \mathrm{RGHBT}$ ). To determine the performance of a less experienced reader and evaluate interobserver variability, all images were independently read by a junior $\mathrm{Gl}$ radiologist with two years experience in pelvic MRI (reader 2, FCHB). The radiologists were blinded for each other's results. Both readers first evaluated the standard T2-weighted images only (further referred to as "standard MRI") and subsequently evaluated the gadofosveset-enhanced images, meanwhile the T2-weighted images remained at their disposal (further referred to as "gadofosveset-MRI"). This reading design is according to the daily clinical practice where contrast enhanced MR images are always evaluated as part of a standard protocol. Each visible node was scored as benign or malignant using a confidence level score $(0=$ definitely benign, $1=$ probably benign, 2 = possibly benign, 3 = probably malignant, and $4=$ definitely malignant). Nodal status was then reported as cNO (no suspicious nodes), cN1 (1-3 suspicious nodes), or cN2 (> 3 suspicious nodes), based on the confidence level scores on standard MRI and gadofosveset-MRI, respectively. Criteria for malignancy 
on standard MRI were size $>5 \mathrm{~mm}$, spiculated or indistinct border and/or heterogeneous signal pattern. ${ }^{12,25}$ Criteria for malignancy on gadofosveset-MRI were low signal intensity and absence of a 'relief sign'. The criteria for gadofosvesetMRI are illustrated in Figure 1.
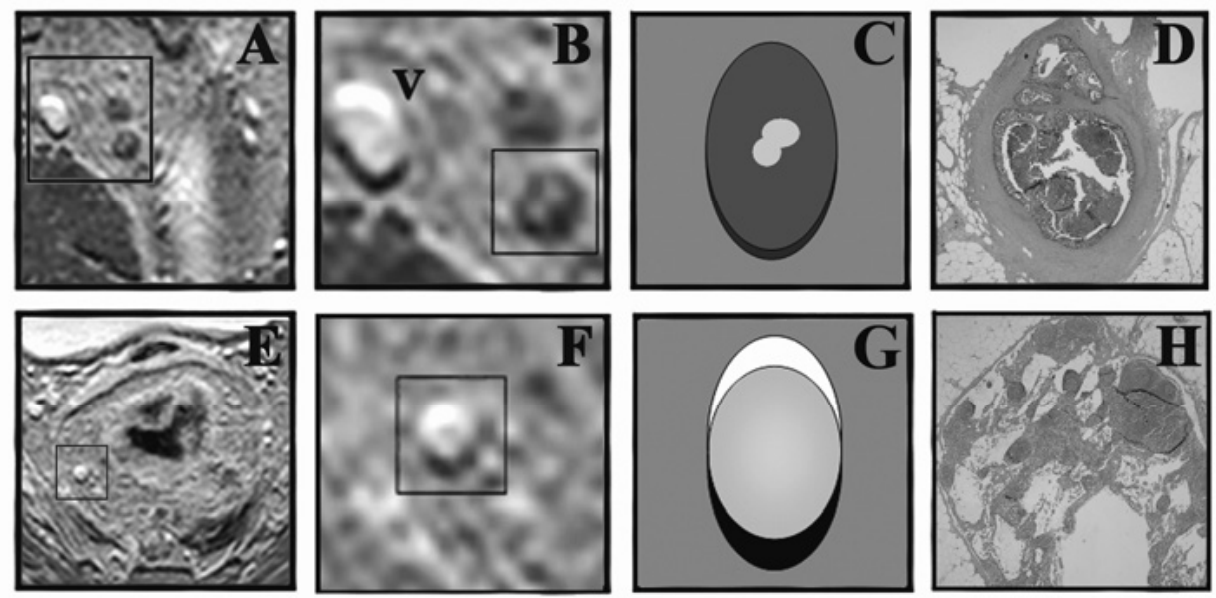

Figure 1. (a-d) Malignant node on gadofosveset-MRI. (a) Two low signal-intensity nodes with a bright adjacent bloodvessel (open rectangle). (b) The node (rectangle) has low signal-intensity and a flat appearance. The vessel (V) has high signal-intensity and a "relief sign", due to accentuation of the water fat shift MR-artefact encircling the vessel. (c) Only a very small part of the node enhances, while the rest remains completely dark. (d) Histology confirmed a malignant node. (e-h) Benign node on gadofosvesetMRI. (e) Small mesorectal node (open rectangle). (f-g) The node has a homogeneous high signal-intensity and a "relief sign", similar to the vessel in (b). (h) Histology confirmed a benign node.

\section{Histopathologic evaluation}

TME surgery was standardised as described by Heald, with en bloc extension outside the mesorectal fascia when required. ${ }^{26}$ To provide accurate matching with the axial MRI, the specimen was sectioned transversely each $5 \mathrm{~mm}$, perpendicular to the tumour axis. Each section was carefully searched for lymph nodes by a dedicated pathologist with fifteen years expertise in colorectal cancer (APB), who was blinded for the MR results. Each node was placed in a marked individual tray and processed according to standard methods. For each node multiple histologic sections were analysed, except for small 1-2 mm nodes in which only one section was assessed. At microscopic examination each node was recorded as benign or malignant. Overall nodal status was then reported as pN0, pN1, or pN2. 


\section{Evaluation of diagnostic performance}

The diagnostic performance of gadofosveset-MRI and standard MRI was analysed a) on a 'lesion by lesion' basis, and b) on a 'patient by patient' basis. The purpose of the lesion by lesion evaluation was to obtain precise validation of imaging findings with the underlying histopathology. This is the first essential step to understand the true value of a new imaging tool. In addition the lesion by lesion results were translated to a patient by patient validation in order to obtain the - clinically more relevant - assessment of the diagnostic performance on a patient basis.

\section{'Lesion by lesion' analysis}

To obtain histologic validation of each individual lymph node, all nodes visualised on MRI were drawn on an anatomical map, which was used as a template for lesion by lesion comparison with histology. Optimal matching between the nodes harvested at pathology and MRI was obtained by side by side comparison of the nodes visible on MRI, the anatomical map and the axially sliced TME specimen. Special attention was given to nodal size and to the location of each node in relation to the tumour, rectal wall, mesorectal fascia, vessels and adjacent nodes. The matching process was performed by a radiological PhD fellow (DMJL).

\section{Statistical analyses}

Statistical analyses were performed using the Statistical Package for the Social Sciences (SPSS, version 16.0, Inc., Chicago, IL). Receiver operator characteristics (ROC) curve analyses were performed. Corresponding areas under the ROC-curve (AUC), sensitivities, specificities, positive predictive values (PPV) and negative predictive values (NPV) with $95 \%$ confidence intervals $(\mathrm{Cl})$ were calculated. For these analyses it had been decided at the start of the study to dichotomise the confidence level scores between 2 (possibly benign) and 3 (probably malignant). Differences in diagnostic performance between standard MRI and gadofosveset-MRI were analysed by comparing the ROC-curves according to the method described by DeLong et al. ${ }^{27}$ In the lesion by lesion analyses, individual nodes cannot not be evaluated as independent observations because nodes are clustered within patients. Therefore, the lesion by lesion analyses were corrected by means of a bootstrap method (number of repetitions 200) with patient number as a resampling cluster. ${ }^{28}$ Diagnostic parameters were separately calculated for group I and group II. $P$-values $<0.05$ were considered statistically significant. Weighted kappa values with quadratic weighting $(0.00-0.20$ poor, 0.21-0.40 fair, 0.41-0.60 moderate, 0.61-0.80 good and 0.81-1.00 excellent agreement) were calculated for evaluation of interobserver agreement. ${ }^{29}$ 


\section{Results}

\section{Patient and treatment characteristics}

All patients tolerated gadofosveset well without adverse events. All MR images were of good quality. For the 42 patients from group II, median time between the restaging $\mathrm{MRI}$ and resection was 17 days (range 1-45).

\section{Patient by patient histologic validation}

At histopathology, a median number of 11 nodes per patient was harvested (range 1-31). In the primary staging group I, 19 patients had a pN0, 5 a pN1 and 2 a pN2 status. In the restaging post-CRT group II, 23 patients had a ypNO, 13 a ypN1 and 6 a ypN2 status.

\section{Lesion by lesion histologic validation}

A lesion by lesion MR-histologic validation of 521 nodes was performed (median 8 nodes per patient, range 1-22), of which 76 nodes were malignant. Median shortaxis diameter of the 521 lymph nodes was $3 \mathrm{~mm}$ (range 2-25). Seventeen of the malignant nodes were retrieved from patients in group I and 59 from group II. All 521 nodes were visualised on gadofosveset-MRI whereas only 269 (52\%) of these 521 nodes were visualised on standard MRI (median 4 nodes per patient, range 0-16).

\section{Overall diagnostic performance and interobserver agreement}

The ROC-curves for the lesion by lesion and patient by patient prediction of nodal status are given in Figure 2. Corresponding accuracy figures and AUCs are provided in Table 1. After addition of gadofosveset-MRI, AUCs for the lesion by lesion prediction of nodal metastases increased from 0.84 to 0.96 for the senior reader 1 $(P<0.001)$ and from 0.85 to 0.93 for the junior reader $2(P=0.03)$. On a patient by patient basis, AUC improved from 0.73 to 0.84 for reader $1(P=0.02)$ and from 0.77 to 0.86 for reader $2(P=0.06)$. Interobserver agreement was good for both standard MRI $(\kappa 0.73)$ and gadofosveset-MRI $(\kappa 0.71)$. For the expert reader 1 , the diagnostic performance remained unchanged during the whole course of the study. The diagnostic performance of the junior reader 2 was initially lower and improved during the study period. Whereas in the first 10 patients he achieved an AUC of 0.79 on a lesion by lesion basis, this improved to $>0.90$ in the later phase of the study. 


\begin{tabular}{|c|c|c|c|c|c|c|c|c|c|c|c|c|c|c|}
\hline \multirow{9}{*}{ 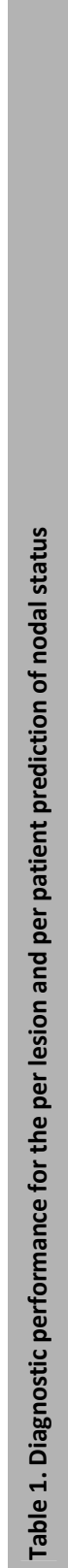 } & \multirow{4}{*}{ 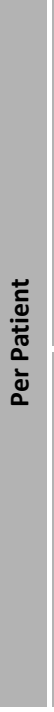 } & \multirow{2}{*}{ 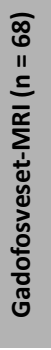 } & $\approx$ & 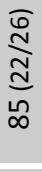 & $\begin{array}{l}m \\
\text { ñ } \\
1 \\
-1\end{array}$ & $\underset{\infty}{\stackrel{\mathcal{N}}{\stackrel{\mathcal{f}}{f}}}$ & $\begin{array}{l}\infty \\
\infty \\
1 \\
m \\
\end{array}$ & 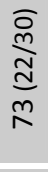 & $\begin{array}{l}\overrightarrow{1} \\
1 \\
\text { ஸे }\end{array}$ & $\frac{\substack{m \\
\stackrel{m}{f}}}{\infty}$ & $\begin{array}{l}n \\
\text { ñ } \\
1 \\
\infty\end{array}$ & $\begin{array}{l}\mathscr{0} \\
\infty \\
0\end{array}$ & $\begin{array}{l}n \\
0 \\
0 \\
1 \\
1 \\
0 \\
0 \\
0\end{array}$ & \multirow{9}{*}{ 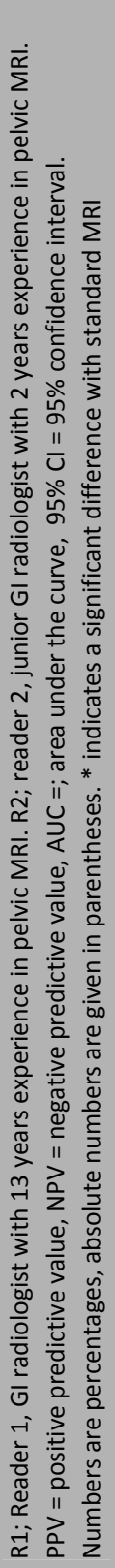 } \\
\hline & & & $\vec{\alpha}$ & 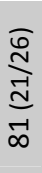 & $\begin{array}{l}\text { ᄋ } \\
1 \\
\hat{6}\end{array}$ & 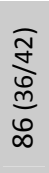 & $\begin{array}{l}\text { के } \\
1 \\
\hat{n}\end{array}$ & 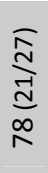 & $\begin{array}{l}\qquad \\
\infty \\
1 \\
\end{array}$ & $\frac{\stackrel{+}{+}}{\stackrel{O}{O}}$ & 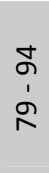 & $\begin{array}{l}\stackrel{*}{*} \\
\stackrel{\infty}{0} \\
\stackrel{0}{*}\end{array}$ & \begin{tabular}{c}
5 \\
0 \\
0 \\
1 \\
\multirow{0}{0}{} \\
0
\end{tabular} & \\
\hline & & $\begin{array}{l}\text { o̊ } \\
\text { "I } \\
\text { 드 }\end{array}$ & $\approx$ & 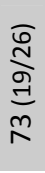 & 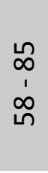 & $\frac{\underset{\mathcal{J}}{\stackrel{\infty}{J}}}{\hat{\sigma}}$ & $\begin{array}{l}\stackrel{N}{N} \\
\text { in }\end{array}$ & 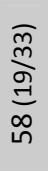 & $\begin{array}{l}\hat{b} \\
\dot{y}\end{array}$ & 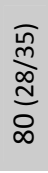 & $\begin{array}{l}\text { ఏ } \\
1 \\
\text { 'ீ }\end{array}$ & $\stackrel{\hat{\imath}}{0}$ & $\begin{array}{l}\infty \\
0 \\
0 \\
0 \\
1 \\
0 \\
0 \\
0 \\
0\end{array}$ & \\
\hline & & 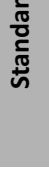 & $\vec{x}$ & 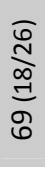 & $\begin{array}{l}\infty \\
\infty \\
1 \\
\text { ஸे }\end{array}$ & $\begin{array}{l}\underset{\mathfrak{d}}{\stackrel{D}{d}} \\
\underset{\hat{v}}{\hat{v}}\end{array}$ & \begin{tabular}{l}
\multirow{N}{1}{} \\
in
\end{tabular} & 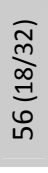 & $\begin{array}{l}\mathscr{b} \\
1 \\
\dot{f}\end{array}$ & $\begin{array}{l}\underset{0}{\infty} \\
\stackrel{m}{\infty} \\
\stackrel{\infty}{\sim}\end{array}$ & $\begin{array}{l}\hat{\infty} \\
1 \\
\hat{\sigma}\end{array}$ & $\stackrel{m}{\stackrel{m}{0}}$ & $\begin{array}{c}n \\
\infty \\
0 \\
0 \\
1 \\
-1 \\
0 \\
0\end{array}$ & \\
\hline & & 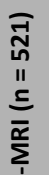 & $\widetilde{\simeq}$ & 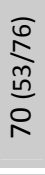 & $\begin{array}{l}\infty \\
1 \\
\dot{0} \\
\qquad\end{array}$ & 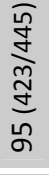 & $\begin{array}{l}\infty \\
\text { o } \\
1 \\
\text { مू }\end{array}$ & $\begin{array}{l}\underset{n}{N} \\
\stackrel{n}{n} \\
\stackrel{-1}{n}\end{array}$ & 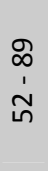 & 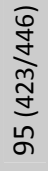 & $\begin{array}{l}\text { o } \\
1 \\
\text { नू }\end{array}$ & $\begin{array}{l}\stackrel{*}{*} \\
\text { ôt } \\
0\end{array}$ & $\begin{array}{l}8 \\
0 \\
-1 \\
1 \\
0 \\
0 \\
0 \\
0\end{array}$ & \\
\hline & 듬 & 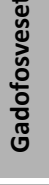 & $\vec{\alpha}$ & 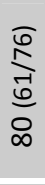 & $\begin{array}{l}8 \\
1 \\
1 \\
-1\end{array}$ & 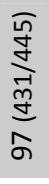 & $\begin{array}{l}\text { ने } \\
\text { nू }\end{array}$ & $\begin{array}{l}\underset{n}{n} \\
\underset{-}{-} \\
\vec{\infty}\end{array}$ & $\begin{array}{l}\text { ๙ } \\
1 \\
\hat{\sigma}\end{array}$ & 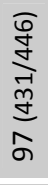 & $\begin{array}{l}\infty \\
\text { م } \\
\text { மn }\end{array}$ & $\begin{array}{l}\text { 苂 } \\
\text { ọ } \\
0\end{array}$ & $\begin{array}{l}\text { on } \\
0 \\
1 \\
\text { m. } \\
0 \\
0\end{array}$ & \\
\hline & ¿ & 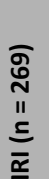 & $\widetilde{\simeq}$ & $\begin{array}{l}\infty \\
\stackrel{\infty}{0} \\
\text { o } \\
\text { gூ }\end{array}$ & $\begin{array}{l}\infty \\
\infty \\
1 \\
\text { 유 }\end{array}$ & 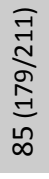 & $\begin{array}{l}\text { के } \\
\text { के }\end{array}$ & 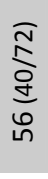 & \begin{tabular}{l}
\multirow{N}{N}{} \\
$\stackrel{1}{m}$ \\
$\hat{m}$
\end{tabular} & 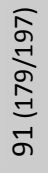 & $\begin{array}{l}\infty \\
\text { ద } \\
1 \\
\infty \\
\infty\end{array}$ & $\begin{array}{l}\stackrel{\llcorner}{\infty} \\
\infty \\
0\end{array}$ & $\begin{array}{l}\text { ñ } \\
0 \\
1 \\
1 \\
0 \\
0 \\
0\end{array}$ & \\
\hline & & 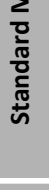 & $\vec{x}$ & $\stackrel{\infty}{\stackrel{\infty}{\lessgtr}}$ & $\begin{array}{l}\text { ○ } \\
1 \\
\tilde{6}\end{array}$ & 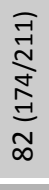 & $\begin{array}{l}\infty \\
\infty \\
1 \\
\wedge\end{array}$ & $\frac{\vec{d}}{\stackrel{D}{J}}$ & $\begin{array}{l}\mathbb{N} \\
1 \\
\hat{m}\end{array}$ & 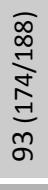 & $\begin{array}{l}\text { के } \\
1 \\
\infty \\
\infty\end{array}$ & 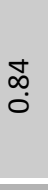 & $\begin{array}{l}0 \\
0 \\
1 \\
0 \\
0 \\
0 \\
0\end{array}$ & \\
\hline & & & & $\stackrel{\geq}{\geq}$ & $\begin{array}{l}\text { 우 } \\
\text { ํํ }\end{array}$ & 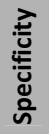 & $\begin{array}{l}\text { ป } \\
\text { ํํ }\end{array}$ & $\frac{a}{a}$ & 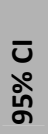 & $\frac{\bar{\alpha}}{2}$ & $\begin{array}{l}\text { च } \\
\text { ํํ }\end{array}$ & $\stackrel{2}{4}$ & $\begin{array}{l}\text { U } \\
\text { ํํ } \\
\text { ஸू }\end{array}$ & \\
\hline
\end{tabular}



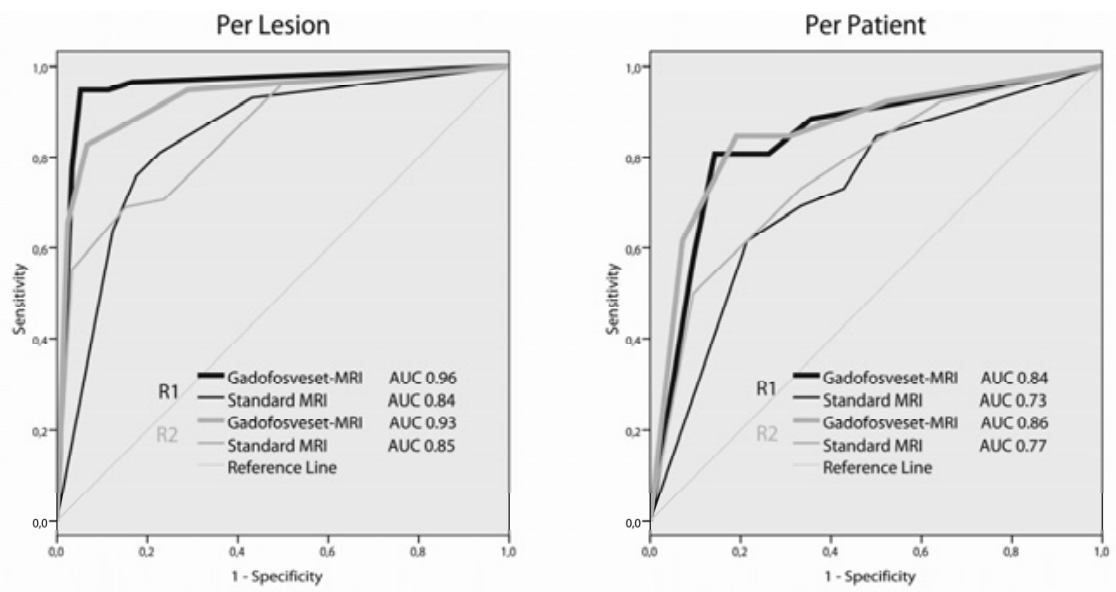

Figure 2. Receiver operator characteristics curves and areas under the curve (AUC) for detection of metastatic lymph nodes using only standard (T2-weighted) MRI and after addition of gadofosvesetenhanced images (gadofosveset-MRI). R1; reader 1, GI radiologist with 13 years experience in pelvic MRI. $\mathrm{R} 2$; reader 2, junior $\mathrm{GI}$ radiologist with 2 years experience in pelvic MRI.
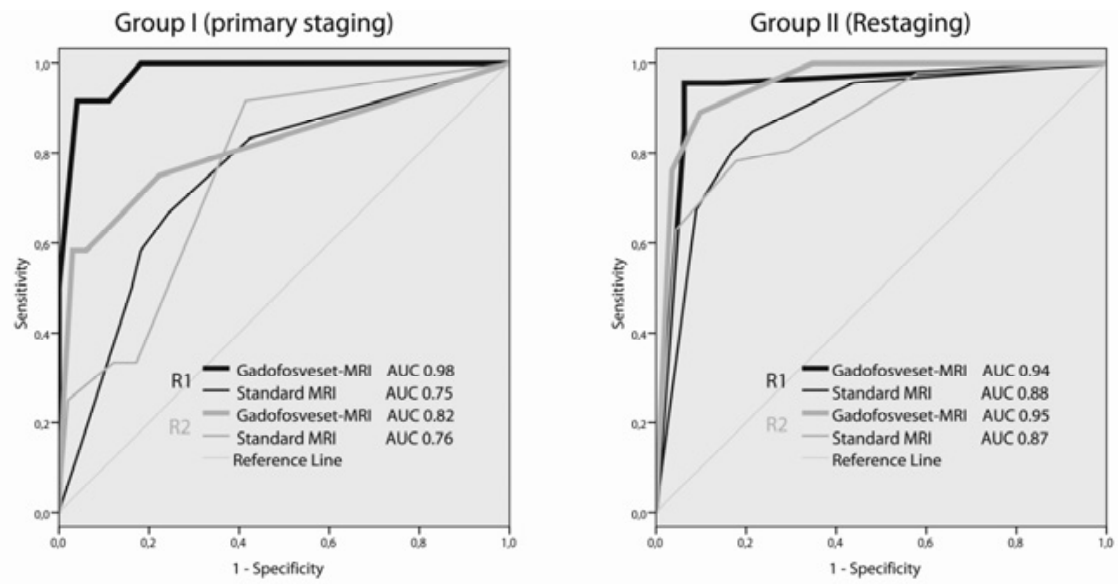

Figure 3. Receiver operator characteristics curves and areas under the curve (AUC) for the per lesion prediction of nodal metastases in the primary staging group I and restaging group II, respectively. R1; reader 1, GI radiologist with 13 years experience in pelvic MRI. R2; reader 2, junior GI radiologist with 2 years experience in pelvic MRI. 


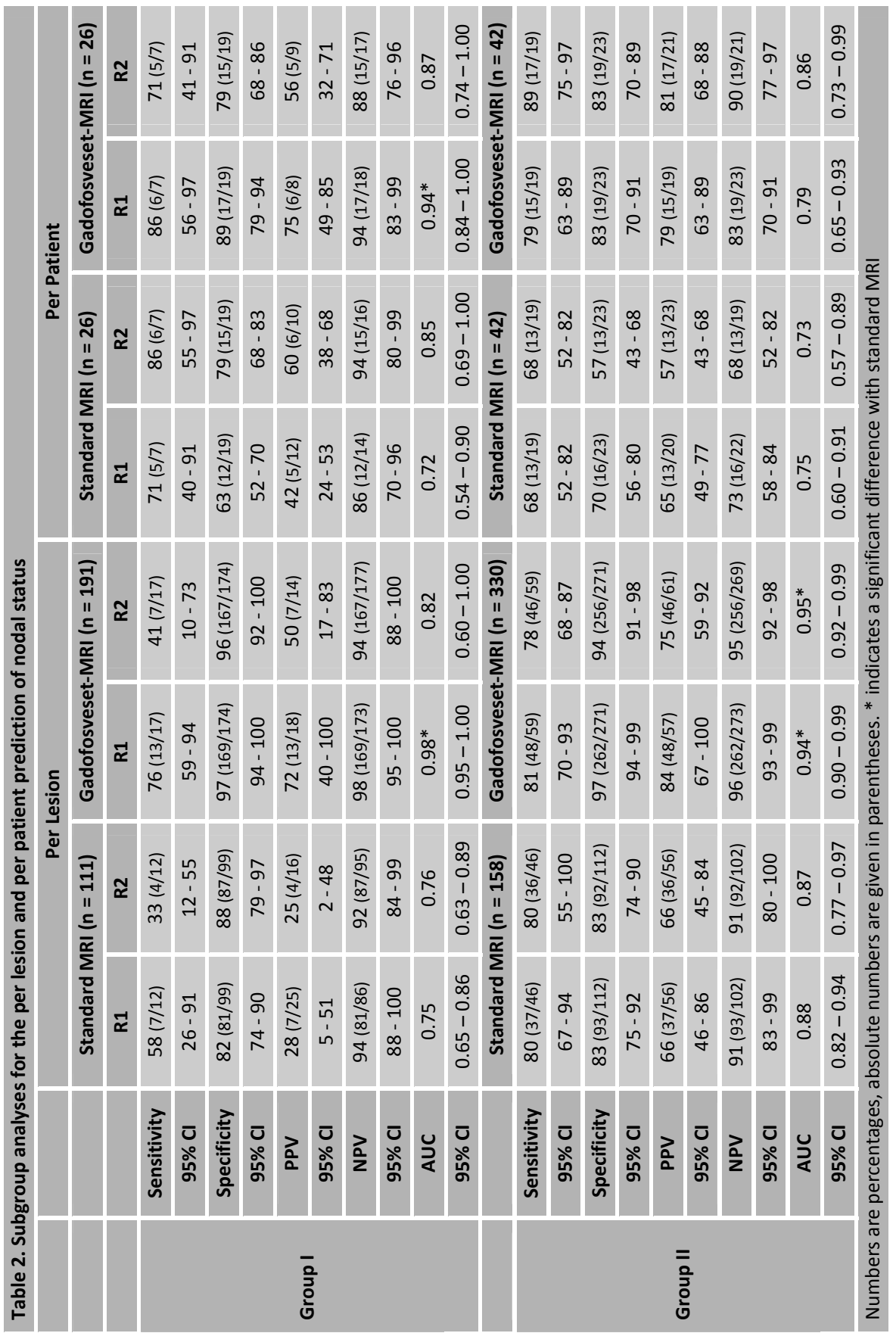




\section{Group I versus group II}

Differences in diagnostic performance between group I and II are displayed in Table 2. The ROC-curves for both subgroups are displayed in Figure 4. In group I, the per lesion AUC improved from 0.75 on standard MRI to 0.98 on gadofosveset-MRI for reader $1(P<0.001)$ and from 0.76 to 0.82 for reader $2(P=0.54)$. On a patient basis, AUC improved from 0.72 to 0.94 for reader $1(P=0.005)$ and from 0.85 to 0.87 for reader $2(P=0.6)$. In group II, the per lesion AUC improved from 0.88 on standard $\mathrm{MRI}$ to 0.94 on gadofosveset-MRI for reader $1(P=0.01)$ and from 0.87 to 0.95 for reader $2(P=0.04)$. On a patient basis AUC changed from 0.75 to 0.79 for reader 1 $(P=0.54)$ and from 0.73 to 0.86 for reader $2(P=0.06)$. Interobserver agreement was good in group II ( $\kappa 0.78$ for both standard MRI and gadofosveset-MRI) but only moderate in group I ( $\kappa 0.60$ and 0.42 , respectively).

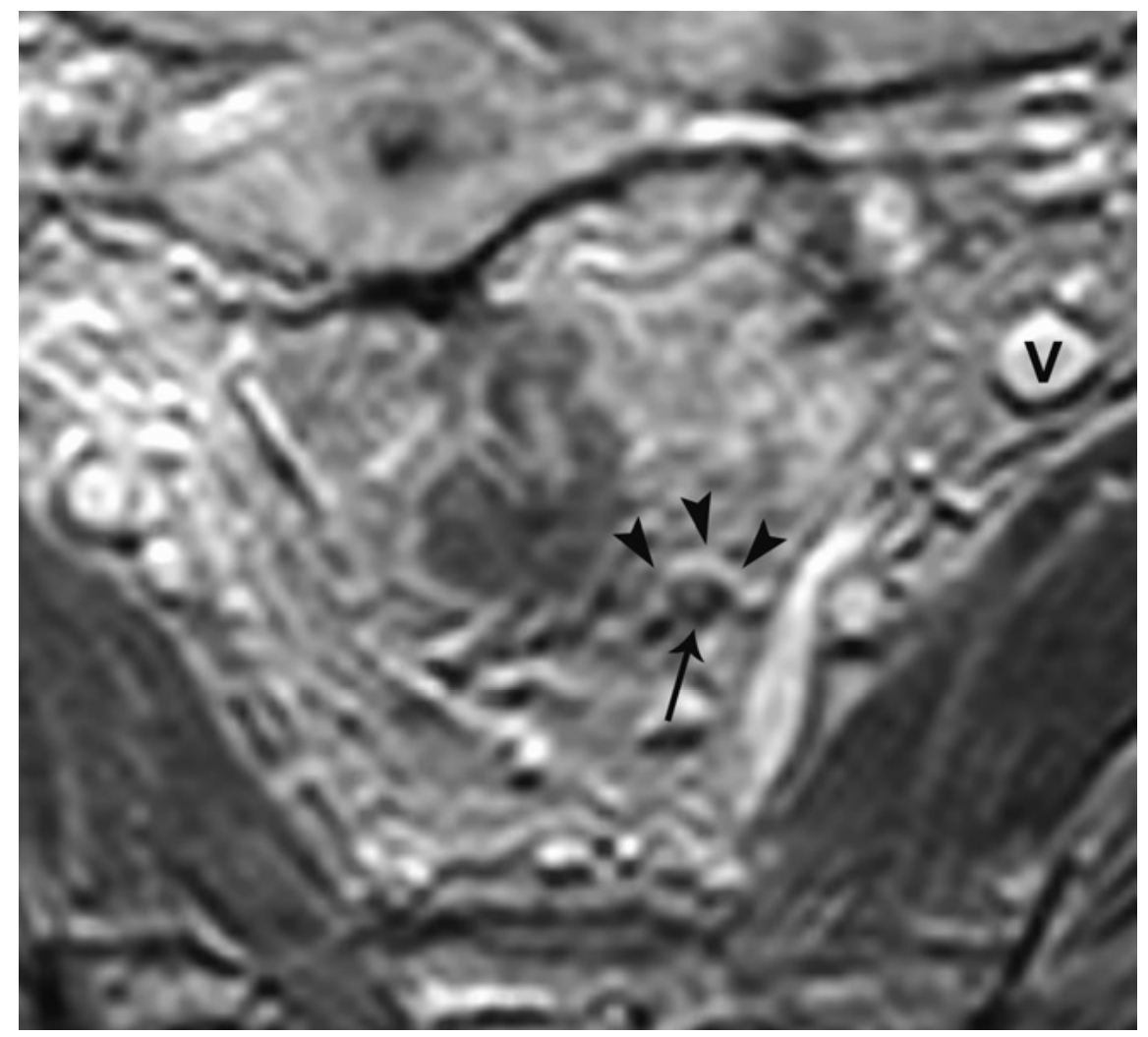

Figure 4. Example of a malignant node (arrow) that was misinterpreted on gadofosveset-MRI by the junior reader. Compared to the strong enhancement of the adjacent blood vessel (V) this malignant node shows no contrast uptake and remains dark. Due to a small vessel that runs anterior to the node (arrowheads), it appears as if this node shows a relief. This pitfall resulted in a false negative assessment by the junior reader. 


\section{Discussion}

The aim of this prospective study was to evaluate the accuracy of gadofosvesetenhanced MRI for nodal staging in rectal cancer with histology as the reference standard. Results were primarily analysed on a lesion by lesion basis, to obtain exact validation of this new contrast with the underlying histology. Gadofosveset-MRI improved the diagnostic performance for nodal staging compared with standard MRI. The benefit of gadofosveset-MRI was most pronounced for the primary staging group I (Figure 4).

The better performance of gadofosveset-MRI can be attributed to both a better detection and characterisation of lymph nodes. With the gadofosvesetenhanced MR images smaller pixels and thus better resolution could be obtained, which may have contributed to the fact that more nodes could be identified than on the lower resolution standard T2W MR images. It is known that with morphological criteria used at standard MRI, the assessment of involved nodes is not reliable, even if higher resolution standard MR images are obtained. ${ }^{7,8}$ This is because the discrimination of metastatic nodes with any morphology based imaging technique goes beyond the level of tumour deposits in small metastatic nodes.

It is in specific the addition of the functional information from the gadofosveset-enhanced $M R$ images to the morphological information from the standard $M R$ images that contributes to the superior performance of gadofosveset-MRI. Even though the exact mechanism is not yet defined, it is clear that normal or reactive lymph nodes show uptake of gadofosveset contrast, inducing a strong increase in signal comparable to that of surrounding blood vessels. $^{17,18}$ Due to this local increase in signal, a ring-shaped MR artefact encircling the lymph node is accentuated, creating the optical impression of a relief effect or 'relief sign' (Figure 1). In metastatic nodes, normal lymphoid tissue is replaced by tumour, preventing the uptake of gadofosveset. Therefore, in malignant nodes, dark areas indicate tumoural deposits. When the whole node is involved, the 'relief sign' will be absent. These criteria - signal enhancement and nodal relief - were highly beneficial for a more accurate distinction between benign and malignant nodes.

Gadofosveset-MRI improved the accuracy of standard MRI both in the primary staging group I and in the restaging group II. The gain in accuracy for the expert reader was, however, less pronounced in group II. This is in concordance with previous reports where nodal restaging with MRI after chemoradiation was already relatively accurate and less benefit was found after administration of lymph node specific MR contrast. ${ }^{30}$ A possible explanation for the good nodal staging results on $\mathrm{MRI}$ after $\mathrm{CRT}$ is that many irradiated nodes disappear and of the 
remaining small nodes over $80 \%$ are sterilised. ${ }^{31}$ Nodes that remain large after CRT are thus more likely to be malignant. Altogether this facilitates a more reliable assessment of the nodal status on restaging MRI based on morphological criteria only.

For the primary staging group I, the expert reader improved significantly with gadofosveset-MRI, both on a per lesion and per patient basis. However, for the junior reader there was no significant improvement in this group and the interobserver agreement with the senior reader was only moderate. This can partly be explained by the observation that in the initial phase of the study a relatively large number of patients were included that did not undergo CRT and thus were assigned to group I. In the beginning of the study, the lack of expertise and confidence of the less experienced reader caused him to misinterpret some small sized malignant nodes as benign (Figure 4), which in the lesion by lesion analyses resulted in an AUC of only 0.79 for the first 10 study patients. After histology revealed the true status of the nodes, he received feedback on his mistakes, which resulted in an obvious positive learning curve during the further period of the study and an improvement in AUC to > 0.90. This finding indicates that - in order for general radiologists to benefit from the addition of gadofosveset and achieve a sufficient level of expertise - efforts should be made to train them and familiarise them with the imaging criteria for gadofosveset-MRI (e.g. by means of expert reader and/or histologic feedback).

To our knowledge, this is the first clinical study evaluating the accuracy of gadofosveset-enhanced MRI for lymph node staging in rectal cancer. The high negative predictive value of more than $95 \%$ on a per lesion and more than $85 \%$ on a per patient basis for gadofosveset-MRI equals that of previous promising reports with the non-FDA approved contrast agent USPIO. ${ }^{13-15,30}$ The PPV is also in the same range as with USPIO; 70-80\%. The advantage of gadofosveset, as opposed to USPIO, is that it is already FDA approved, proven safe for human use and available on the market for clinical use. It will therefore be easier to implement in clinical practice, when the results of our study are confirmed in larger studies.

Our study is limited in that the total number of patients - particularly in the primary staging group $\mathrm{I}$ - is limited, which is reflected in the wide confidence intervals in the per patient analyses. Our primary intention, however, was to evaluate this novel MR contrast agent by means of a per node validation with histopathology as the reference standard, which (as previously mentioned) is the first essential step to take when introducing a new imaging tool. Our preliminary patient by patient results show encouraging results towards improved performance when using gadofosveset-enhanced MRI, although no firm conclusions can be drawn based on the current patient numbers. To establish the true clinical value of 
gadofosveset-MRI in determining lymph node status, larger validation studies with evaluation on a per patient basis are required.

\section{Clinical impact}

So far, clinicians have been reluctant to make decisions based on the nodal staging by imaging because of its insufficient accuracy. A reliable preoperative staging of nodes could however benefit patients with rectal cancer. Truly node negative patients have a low risk for local recurrence and most of these patients do not benefit from neoadjuvant therapy. ${ }^{3}$ Small node negative tumours can even be treated with a local excision, further reducing functional morbidity and costs. ${ }^{19}$ With increasingly effective preoperative chemoradiation, many initially involved nodes are sterilised, and some of these 'good responders' could be considered for local excision or - even more controversial - omission of surgery when in complete remission. ${ }^{20-22}$ For all of these treatment options an accurate assessment of nodal status is mandatory.

In conclusion, this study shows that MRI using a new gadolinium based contrast agent (gadofosveset) is promising for predicting lymph node metastases in rectal cancer. Addition of gadofosveset contrast to a standard rectal MRI could potentially improve the accuracy of MRI for staging rectal cancer nodes. Node negative patients can be identified with a high negative predictive value, limiting the potential risk for understaging and undertreatment. In addition, the positive predictive value is fairly good, resulting in acceptable overstaging and overtreatment. Gadofosveset should therefore be further studied as a potentially promising lymph node specific contrast agent. When our lesion by lesion findings are confirmed by larger studies with evaluation on a patient by patient basis, the use of gadofosveset-MRI could allow for better individual tailoring of treatment for rectal cancer patients. 


\section{References}

1 Sauer R, Becker H, Hohenberger W, et al. Preoperative versus postoperative chemoradiotherapy for rectal cancer. N Engl J Med 2004; 351:1731-40

2 Nagtegaal ID, Quirke P. What is the role of the circumferential margin in the modern treatment of rectal cancer? J Clin Oncol 2008; 26:303-12

3 Kapiteijn E, Marijnen CA, Nagtegaal ID, et al. Preoperative radiotherapy combined with total mesorectal excision for resectable rectal cancer. N Engl J Med 2001; 345:638-46

4 Gosens MJ, van Krieken JH, Marijnen CA, et al. Improvement of staging by combining tumor and treatment parameters: the value for prognostication in rectal cancer. Clin Gastroenterol Hepatol 2007; 5:997-1003

5 MERCURY Study Group: Diagnostic accuracy of preoperative magnetic resonance imaging in predicting curative resection of rectal cancer: prospective observational study. BMJ 2006;333:779

6 Beets-Tan RG, Beets GL, Vliegen RF, et al. Accuracy of magnetic resonance imaging in prediction of tumor-free resection margin in rectal cancer surgery. Lancet 2001;357:497-504

7 Lahaye MJ, Engelen SM, Nelemans PJ, et al. Imaging for predicting the risk factors--the circumferential resection margin and nodal disease--of local recurrence in rectal cancer: a metaanalysis. Semin Ultrasound CT MR 2005;26:259-68

8 Bipat S, Glas AS, Slors FJ, Zwinderman AH, Bossuyt PM, Stoker J. Rectal cancer: local staging and assessment of lymph node involvement with endoluminal US, CT, and MR imaging--a meta-analysis. Radiology 2004;232:773-83

9 Guillem JG, Díaz-González JA, Minsky B, et al. cT3NO rectal cancer: potential overtreatment with preoperative chemotherapy is warranted. J Clin Oncol 2008;26:368-73

10 Wang C, Zhou Z, Wang Z, et al. Patterns of neoplastic foci and lymph node micrometastasis within the mesorectum. Langenbecks Arch Surg 2005:390:312-18

11 Dworak O: Number and size of lymph nodes and node metastases in rectal carcinomas. Surg Endosc 1989;3:96-99

12 Brown G, Richards CJ, Bourne MW, et al. Morphologic predictors of lymph node status in rectal cancer with use of high-spatial-resolution MR imaging with histopathologic comparison. Radiology 2003;227:371-77

13 Lahaye MJ, Engelen SM, Kessels AG, et al. USPIO-enhanced MR imaging for nodal staging in patients with primary rectal cancer: predictive criteria. Radiology 2008;246:804-11

14 Koh D-M, Brown G, Temple L, Raja et al. Rectal Cancer: Mesorectal Lymph Nodes at MR Imaging with USPIO versus Histopathologic Findings--Initial Observations. Radiology 2004;231:91-99

15 Will O, Purkayastha S, Chan C, et al. Diagnostic precision of nanoparticle-enhanced MRI for lymphnode metastases: a meta-analysis. Lancet Oncol 2006;7:52-60

16 Gadofosveset: MS 325, MS 32520, Vasovist, ZK 236018. Drugs R D 2004;5:339-42

17 Herborn CU, Lauenstein TC, Vogt FM, Lauffer RB, Debatin JF, Ruehm SG. Interstitial MR lymphography with MS-325: characterization of normal and tumor-invaded lymph nodes in a rabbit model. Am J Roentgenol 2002;179:1567-1572

18 Lahaye MJ, Beets GL, Engelen SME, et al. Gadovosfeset Trisodium (Vasovist ) enhanced MR lymph node detection: initial observations. The Open Magnetic Resonance Journal 2009;2:1-5

19 Middleton PF, Sutherland LM, Maddern GJ. Transanal endoscopic microsurgery: a systematic review. Dis Colon Rectum 2005;48:270-84

20 Lezoche G, Baldarelli M, Guerrieri M, et al. A prospective randomized study with a 5-year minimum follow-up evaluation of transanal endoscopic microsurgery versus laparoscopic total mesorectal excision after neoadjuvant therapy. Surg Endosc 2008;22:352-58

21 Borschitz T, Wachtlin D, Möhler M, Schmidberger H, Junginger T. Neoadjuvant chemoradiation and local excision for T2-3 rectal cancer. Ann Surg Oncol 2008;15:712-20 
22 Habr-Gama A, Perez RO, Proscurshim I, et al. Patterns of failure and survival for nonoperative treatment of stage c0 distal rectal cancer following neoadjuvant chemoradiation therapy. J Gastrointest Surg 2006;10:1319-28

23 Shellock FG: The reference manual for magnetic resonance safety, implants and devices (ed 2010). Los Angeles, CA, Biomedical Research Publishing Company

24 Marijnen CAM, Nagtegaal ID, Klein Kranenbarg E, et al. No downstaging after short-term preoperative radiotherapy in rectal cancer patients. J Clin Oncol 2001;7:1976-84

$25 \mathrm{Kim} \mathrm{JH}$, Beets GL, Kim MJ, Kessels AG, Beets-Tan RG. High-resolution MR imaging for nodal staging in rectal cancer: are there any criteria in addition to the size? Eur J Radiol 2004;52:78-83

26 Heald RJ: A new approach to rectal cancer. Br J Hosp Med 1979;22:277-281, 1979

27 DeLong ER, DeLong DM, Clarke-Pearson DL. Comparing the areas under two or more correlated receiver operating characteristic curves: a nonparametric approach. Biometrics 1988;44:837-845

28 Efron B, Tibshirani RJ. An introduction to the bootstrap (ed 1). New York, Chapman \& Hall, 1993

29 Cohen J. Weighted Kappa: nominal scale agreement with provision for scaled disagreement or partial credit. Psychol Bull 1968;70:213-230

30 Lahaye MJ, Beets GL, Engelen SME, et al. Locally advanced rectal cancer: MR imaging for restaging after neoadjuvant radiation therapy with concomitant chemotherapy. Part II. What are the criteria to predict involved lymph nodes? Radiology 2009;252:71-80, 2009

31 Koh DM, Chau I, Tait D, et al. Evaluating mesorectal lymph nodes in rectal cancer before and after chemoradiation using thin-section T2-weighted magnetic resonance imaging. Int J Radiat Oncol Biol Phys 2008; 71:456-61 

FOLLOW-UP 



\section{LONG-TERM FOLLOW-UP FEATURES ON RECTAL MRI DURING A WAIT-AND-SEE APPROACH AFTER A CLINICAL COMPLETE RESPONSE IN PATIENTS WITH RECTAL \\ CANCER TREATED WITH CHEMORADIOTHERAPY}

\section{CHAPTER}

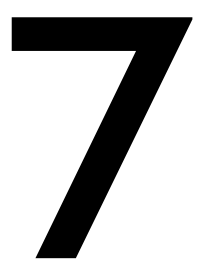

DMJ Lambregts M Maas FCH Bakers VC Cappendijk G Lammering GL Beets RGHBeets-Tan

Published in: Diseases of the Colon and Rectum 2011; 54(12):1521-28 


\section{Abstract}

\section{Aim}

The 'wait-and-see' policy instead of standard surgery for rectal cancer patients who undergo a complete tumour regression after chemoradiation treatment is highly controversial. It is not clear yet how patients should be monitored once they are managed non-operatively and whether follow-up by MRI has any potential role. This study aimed to describe the rectal wall MRI morphology during short-term and longterm follow-up in patients with a clinical complete tumour response undergoing a wait-and-see policy without surgical treatment.

\section{Materials \& Methods}

As part of an observational study in our center, a cohort of 19 carefully selected patients with a clinical complete response after chemoradiation was managed with a wait-and-see policy and followed regularly (every 3-6 mo) by clinical examination, endoscopy with biopsies, and a rectal MRI. The MR morphology of the tumour bed was studied on the consecutive MRI examinations. The primary outcome measured was the morphology of the tumour bed on the consecutive MRI examinations performed during short-term (<=6 mo) and long-term (>6 mo) follow-up.

\section{Results}

Patients with a complete tumour response after chemoradiation presented with either a normalised rectal wall (26\%) or fibrosis (74\%). In the latter group, 3 patterns of fibrosis were observed (full-thickness, minimal, or spicular fibrosis). The morphology patterns of a normalised rectal wall or fibrosis remained consistent during long-term follow-up in 18 of 19 patients. One patient developed a small, endoluminal recurrence, which was salvaged with transanal endoscopic microsurgery. In $26 \%$ of patients, an edematous wall thickening was observed in the first months after chemoradiation, which gradually decreased during long-term follow-up. Median follow-up was 22 months (range, 12-60).

\section{Conclusions}

Four MR patterns of a persistent complete response of rectal cancer after chemoradiation were identified. These MR features can serve as a reference for the follow-up in a wait-and-see policy. 


\section{Introduction / Clinical background}

The standard treatment for patients with locally advanced rectal cancer consists of a long course of neoadjuvant chemoradiation (CRT) followed by surgical resection. ${ }^{1}$ In recent years, there has been a highly controversial trend to opt for more conservative, organ saving treatments in patients who show a very good response to neoadjuvant treatment. ${ }^{2-4}$ Observational cohort studies have shown that a "wait-and-see" policy can be a good alternative to surgery without compromising the outcome in carefully selected patients with clinical evidence of a complete response of both the tumour and lymph nodes. ${ }^{2,5,6}$ However, several issues need to be addressed in this clinical context. First, it is still unclear which clinical selection method is the most accurate for the identification of clinical complete responders after CRT and whether and how imaging can be of additional benefit. For this purpose, ongoing studies are evaluating the potential role of tumour markers, endoscopy with or without biopsies, and morphological and functional imaging techniques. ${ }^{7-13}$ Second, there is no evidence yet as to how and how frequently patients should be monitored once they are managed nonoperatively. Third, even if imaging proves to be beneficial for the selection and monitoring of the complete responders, information on morphological changes that may occur during long-term follow-up is lacking, making it difficult to understand which of these changes could be indicative of tumour regrowth.

The aim of this study was to establish (changes in) the MR morphology of the rectum in patients with a complete tumour response during nonoperative management and follow-up with MRI. The study describes the evolution of the rectal wall morphology on MRI during short-term (<=6 mo) and long-term ( $>6 \mathrm{mo}$ ) followup in patients with a clinical complete response who chose a wait-and-see policy in our unit. To the best of our knowledge, this is the first report on this subject. We aim to share the MR characteristics of these patients at the moment of their selection (6-8 weeks after completion of CRT) and at regular MRIs performed during their clinical follow-up.

\section{Patients and Methods}

\section{Study design}

In our unit patients with locally advanced rectal cancer are routinely treated with a preoperative long course of neoadjuvant chemoradiation (50.4 Gy radiation + $2 \times 825 \mathrm{mg} / \mathrm{m}^{2} / \mathrm{d}$ capecitabine) when on primary staging MRI there is (a) a T3-4 tumour with a threatened or involved mesorectal fascia, (b) > 3 metastatic 
lymph nodes, and/or (c) tumour in the distal rectum $(\leq 5 \mathrm{~cm}$ from the anorectal junction). Since 2004, in addition to standard rectal cancer surgery, an alternative wait-and-see approach is offered to rectal cancer patients who show a clinical complete response (ycTONO) after CRT. The response after chemoradiation was assessed with a combination of MRI, endorectal ultrasound, digital examination and endoscopy with biopsies. Patients were identified as complete responders when there was (a) substantial tumour downsizing with no residual tumour or with fibrosis only on restaging MRI performed 6-8 weeks after CRT, (b) no suspicious nodes on post-chemoradiation $\mathrm{MRI}$, (c) no residual tumour at endoscopy with negative biopsies, and (d) no palpable tumour at digital rectal examination when palpable at primary presentation. The evaluation of response and the diagnosis of a clinical complete response was established 6 to 8 weeks after completion of CRT. Patients with a complete response who were offered a wait-and-see policy were well informed on the controversial and investigational nature of this nonoperative approach and the associated benefits and risks. All but one of the patients who were offered it chose the wait-and-see policy, mainly because of the opportunity to avoid major surgery and the potential risk of a permanent colostomy. Patients were monitored every 3 months during the first year and every 6 months during the following years with clinical examination, serial CEA measurements, CT for evaluation of distant metastases, and endoscopy and rectal MRI for evaluation of local recurrence.

Table 1. 'Standard' rectal MRI protocol: T2W FSE in 3 planes

\begin{tabular}{|c|c|}
\hline Repetition time / echo time (ms) & $8456 / 130$ \\
\hline Number of slices & 30 \\
\hline Slice thickness & 3 \\
\hline Slice gap & 2 \\
\hline Flip angle (degrees) & 90 \\
\hline In plane resolution ( $\mathrm{mm} \times \mathrm{mm}$ ) & $0.78 \times 1.14$ \\
\hline Echotrain length & 25 \\
\hline Number of signal averages (NSA) & 6 \\
\hline Acquisition time (min) & 6.03 \\
\hline \multicolumn{2}{|c|}{$\begin{array}{l}\text { Patients were imaged at } 1.5 \mathrm{~T} \text { (Intera; Philips Medical Systems, Best, The Netherlands), using a phased } \\
\text { array surface coil. Patients did not receive a bowel preparation or spasmolytics. At primary staging, the } \\
\text { axial images were angled perpendicular to the tumour axis as identified on the sagittal MRI. The } \\
\text { coronal images were angled parallel to the tumour axis. For the follow-up MRIs, the imaging planes } \\
\text { from the primary staging MRI were repeated in order to obtain identical imaging planes. }\end{array}$} \\
\hline
\end{tabular}




\section{MR imaging and image evaluation}

Patients underwent a standard pelvic MRI (1.5T) as routinely used for the clinical staging of rectal cancer patients. The protocol is described in Table 1. As part of ongoing research, patients underwent an additional diffusion-weighted $\mathrm{MR}$ sequence (STIR fatsupression, b-values 0,500,1000 s/mm², TR/TE 4829/70 msec, EPI factor 53, 4 NSA, $2.50 \times 3.11 \times 5.00 \mathrm{~mm}$ acquisition voxel size, 50 slices, 10.37 minutes acquisition time). For each patient, the morphology of the tumour was evaluated on the primary staging MRI. The aspect of the tumour bed after chemoradiation was examined on the first restaging MRI performed 6-8 weeks after completion of CRT and on each consecutive follow-up MRI (every 3 months during the first year of follow-up and every 6 months during the following years). All MR images were evaluated by an experienced pelvic MR reader, who recorded the size/volume, shape, aspect of the border and signal intensity of the tumour area on the T2-weighted MR images. Images were compared with the earlier follow-up images to establish changes over time. On high b-value diffusion-weighted MRI, the presence (suggestive of tumour) or absence (suggestive of complete response) of high signal intensity within the tumour bed was recorded (Figure 1). ${ }^{14,15}$
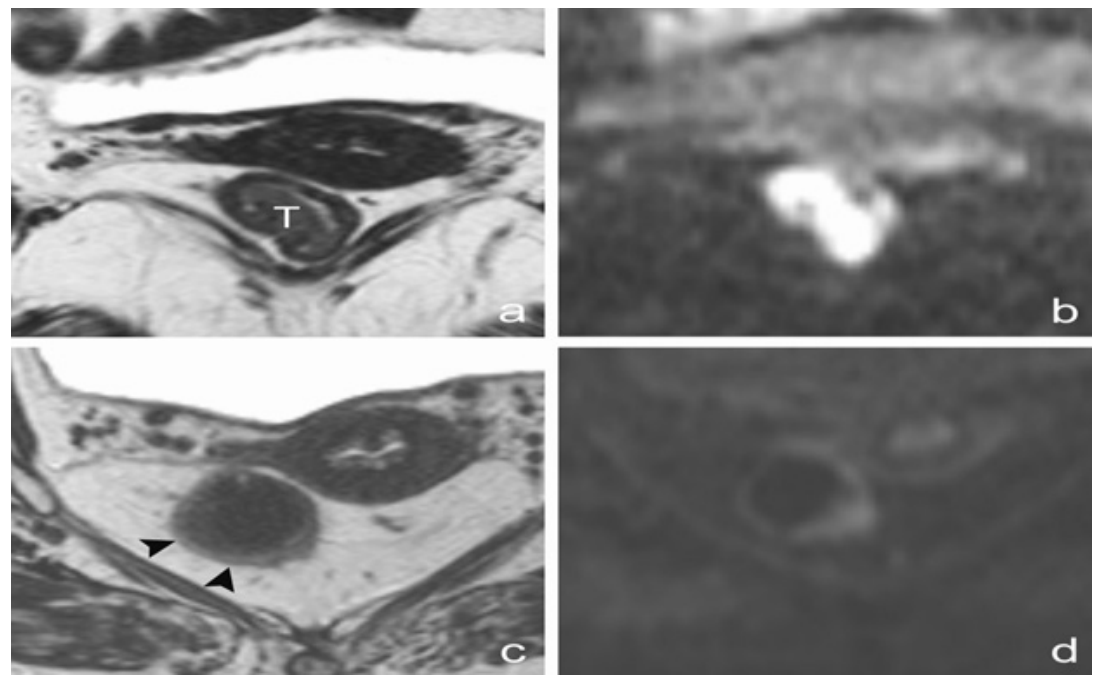

Figure 1. Axial T2-weighted (a,c) and high b-value (b1000) diffusion-weighted (b, d) images of a patient before and after chemoradiation treatment. Before chemoradiation, a tumour $(T)$ showing intermediate signal intensity is visible on T2-weighted MRI (a). On the corresponding diffusion image (b) the tumour can be identified as a clearly defined area of high signal intensity compared to the low signal intensity of the surrounding tissues. After chemoradiation, a normalised two-layered rectal wall (arrowheads) is visible on T2-weighted MRI (c) and no residual tumour can be detected. On the corresponding diffusion image (d) there is no remaining high signal intensity and the patient was identified as having undergone a complete response. 


\section{Results}

\section{The study group}

So far, nineteen patients have chosen the non-operative approach and are being monitored within a defined study protocol. $^{5}$ The initial tumour stage at primary presentation consisted of cT1-2 in five patients, cT3 in twelve patients and cT4 in two patients. The cN stage was N0 in five patients, N1 in four patients and N2 in ten patients. In thirteen patients the initial tumour was located $<5 \mathrm{~cm}$ from the anorectal junction, in five patients it was located $5-10 \mathrm{~cm}$ from the anorectal junction and in one patient $>10$ $\mathrm{cm}$ from the anorectal junction. At the time of writing, the follow-up period ranged between 12 and 60 months (median follow-up 22 months) and the number of follow-up MR examinations ranged accordingly between 3 and 11 (Table 2).
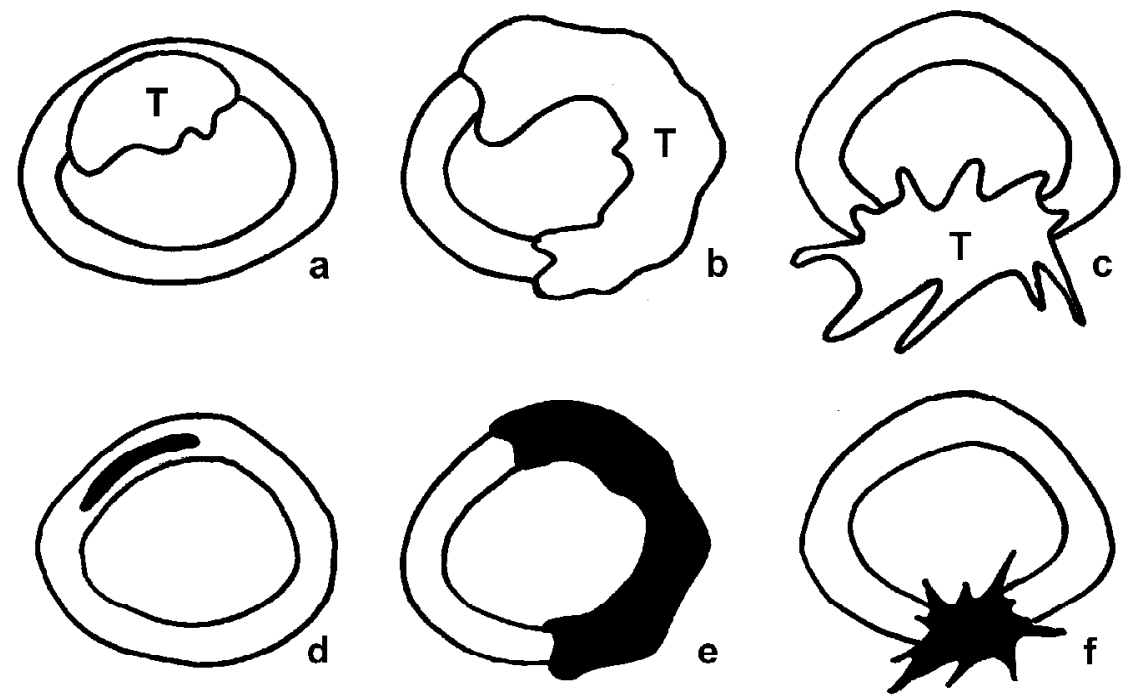

Figure 2. Patterns of fibrosis. Schematic view of three types of tumours (T) before treatment (a-c) and the corresponding patterns of fibrosis ( $d-f)$ that were frequently observed after chemoradiation treatment. In patients with relatively small, often polypoid tumours (a), minimal areas of residual fibrosis were mostly observed after CRT, which remained limited to the bowel wall (d: 'minimal fibrosis'). In patients with more bulky tumours, mostly extending over $50 \%$ of the rectal circumference (b), the tumours often underwent massive fibrotic changes, but showed a relatively small decrease in volume (e: 'full thickness fibrosis'). In patients with more irregular, spiculated tumour types (c), varying degrees of volume reduction were observed. The fibrotic residue remained ill-defined and spiculated, similar to the aspect of the primary tumour (f: 'irregular fibrosis'). 

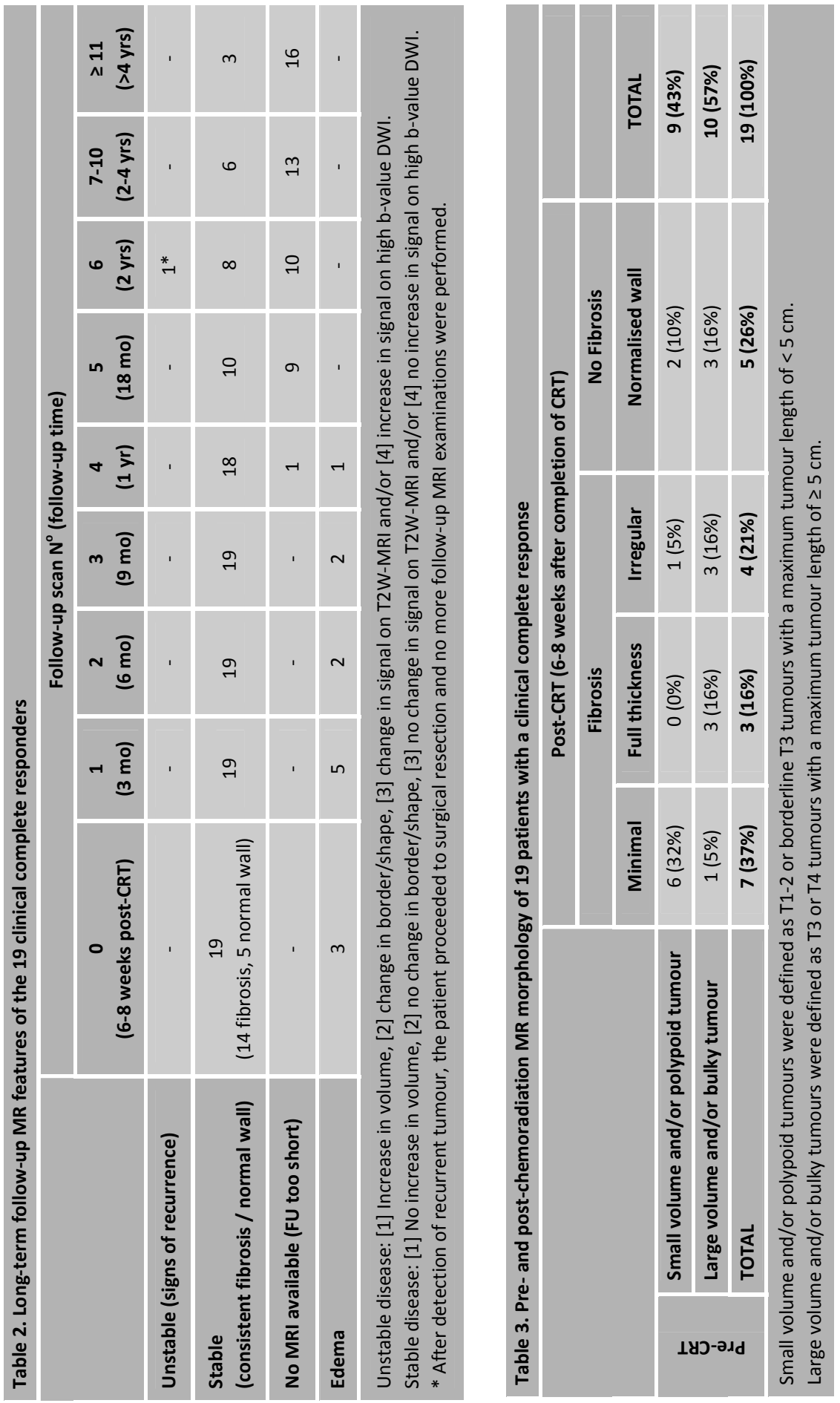


\section{MRI findings at short-term ( $\leq 6$ months) follow-up}

Generally, four distinct groups of MR features could be identified at short-term follow-up (table 3). In 14 of the 19 patients (74\%), at least some area of dark signal intensity was shown in the irradiated tumour bed on the first MRI performed 6 to 8 weeks after completion of CRT, which is suggestive for fibrosis. Three patterns of fibrosis could be distinguished, the aspects of which depended on the initial tumour morphology at primary MR staging (Figure 2). The fourth pattern, which was observed in 5 of the 19 patients (26\%), consisted of a completely normalised, twolayered rectal wall (Figure 1). At endoscopy, patients typically showed either a normalised rectal wall or a small white area of scar tissue at the location of the former tumour (Figure 3).
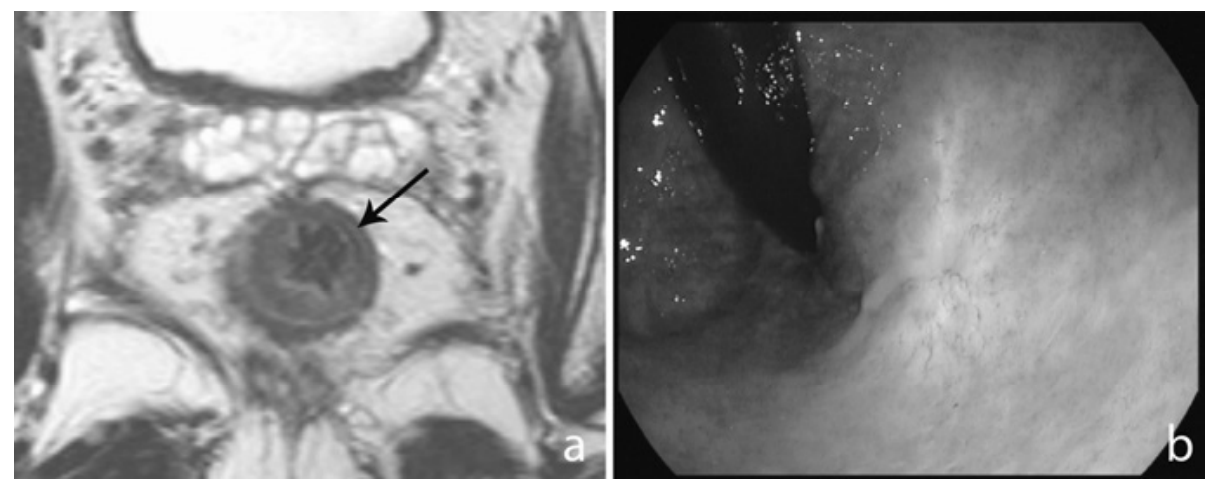

Figure 3. Typical white scar at endoscopy. Axial MR image (a) and corresponding endoscopic view (b) of a patient, managed with a wait-and-see policy, at 1 year after completion of chemoradiation treatment. On the MRI a small fibrotic rim is visible within the rectal wall (arrow). At the corresponding endoscopy, a typical area of white scar tissue is visualised at the location of the former tumour.

\section{Pattern 1: 'Minimal' fibrosis}

Relatively small volume and/or polypoid tumours (cT1-2 or borderline T3, tumour length $<5 \mathrm{~cm}$ ) predominantly showed a marked decrease in tumour volume. On the restaging scan performed 6-8 weeks after CRT these tumours typically regressed to a minimal area of residual fibrosis that remained restricted within the layers of the rectal wall, causing minimal local thickening of the rectal wall (Figure 4). This type of 'minimal' fibrosis was observed in 7 of 19 (37\%) of the patients.

\section{Pattern 2: 'Full-thickness' fibrosis}

In three patients (16\%) with relatively bulky tumour types (T3-4, tumour length $\geq 5 \mathrm{~cm}$ ), often growing over a large circumference of the rectal lumen, only a limited reduction in tumour size was observed. The shape of the irradiated tumour bed 
followed that of the initial tumour and remained relatively consistent during the follow up period. The initially grey (isointense) tumour on MRI became fully dark (hypointense), which suggests fibrotic transformation of the tumour. These cases were classified as a 'full-thickness' fibrotic transformation of the primary tumour (Figure 5).
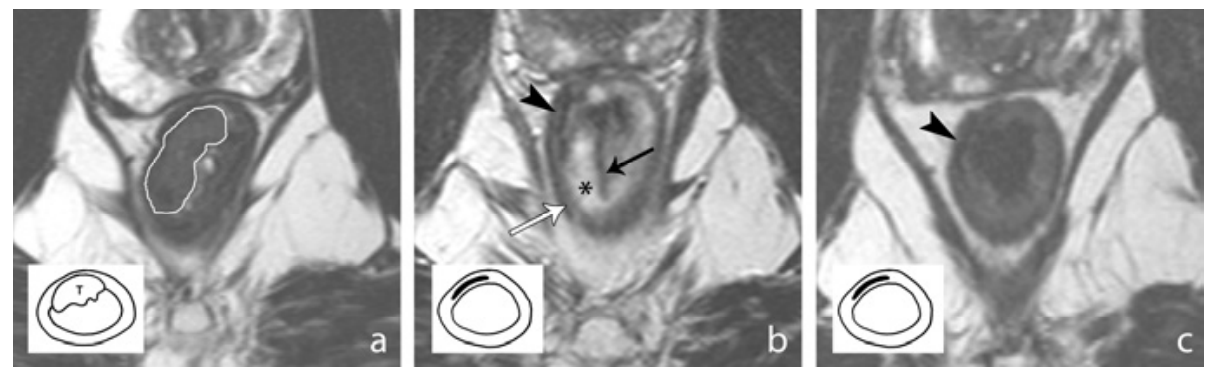

Figure 4. 'Minimal fibrosis' during short-term and long-term follow-up. Axial MR images of a rectal cancer patient before chemoradiation treatment (a), 6-8 weeks after completion of chemoradiation treatment (b) and at two years follow-up (c). A small polypoid tumour is visible in the distal rectum before treatment (a). After chemoradiation (b) the tumour has disappeared. The rectal wall now shows some edematous thickening, which can be recognised as a three layered pattern, consisting of the inner dark layer of mucosa (black arrow), a bright layer of submucosa $\left({ }^{*}\right)$ and the outer dark layer of the muscular rectal wall (white arrow). There is also a small residual dark area of fibrosis (black arrowhead). At two years follow-up (c), the edematous thickening has disappeared and the bowel wall has turned normal, except for at the site of the tumour bed (black arrowhead), which remained dark, indicative of fibrosis.
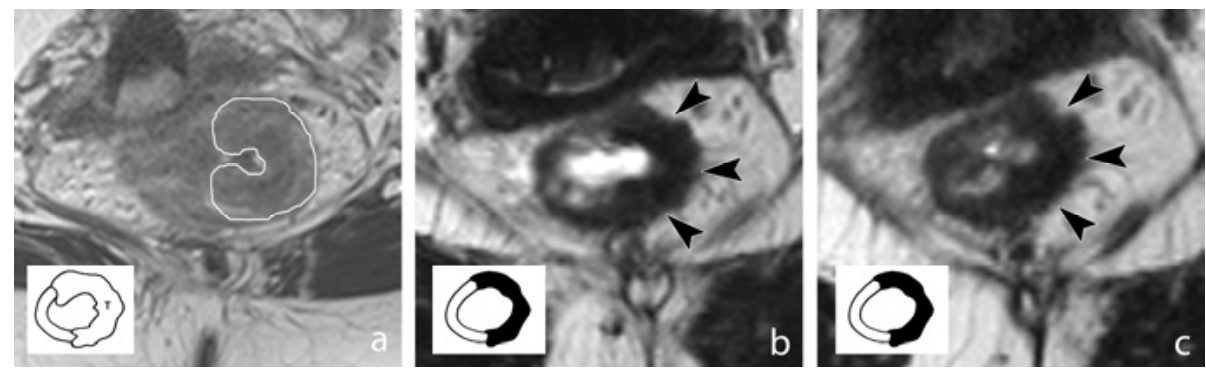

Figure 5. 'Full-thickness fibrosis' during short-term and long-term follow-up. Axial MR images of a rectal cancer patient before chemoradiation treatment (a), 6-8 weeks after completion of chemoradiation treatment (b) and at $1 \frac{1}{2}$ year of follow-up (c). Before treatment (a) there is a tumoural thickening extending over $\pm 50 \%$ of the rectal circumference. After chemoradiation (b), a small volume reduction has occurred and the shape of the wall thickening remained unchanged. However, the tumour has turned fully dark indicative of fibrosis (black arrowheads). At $1 \frac{1}{2}$ year follow-up (c) the fibrotic area remained unchanged and showed only a very subtle further decrease in volume. 


\section{Pattern 3: 'Irregular' fibrosis}

In four patients (21\%) with irregularly shaped, spiculated and ill-defined tumours (T3-4, tumour length $\geq 5 \mathrm{~cm}$ ), varying degrees of volume reduction were observed. In some of these patients there was remarkable reduction in tumour volume, whereas in others there was only small decrease in volume. In all four patients, however, the tumour bed turned dark because of fibrosis. The initial tumour shape with irregular and spiculated borders persisted (Figure 6).
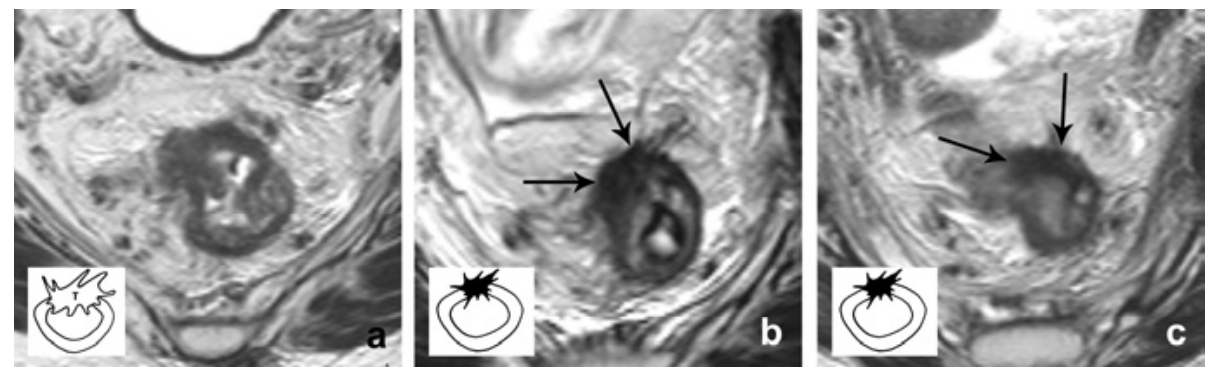

Figure 6. 'Irregular fibrosis' during short-term and long-term follow-up. Axial MR images of a rectal cancer patient before chemoradiation treatment (a) 6-8 weeks after completion of chemoradiation treatment (b) and at 1 year of follow-up (c). Before treatment (a) there is a tumour in the mid-rectum with irregular borders and spiculations into the mesorectal fat. After chemoradiation (b) there is hardly any volume reduction but an area of hypointense signal intensity, suggestive of fibrosis (arrows), has replaced the initially isointense tumour area. This fibrosis follows the shape of the primary tumour with irregularly shaped borders and spiculations. At 1 year of follow-up (c), the shape of the fibrotic thickening persisted and only showed minimal shrinkage.

\section{Pattern 4: Normalised rectal wall}

In $26 \%$ of the patients the rectal wall was completely normal at MRI performed 6-8 weeks after CRT and no residual tumour mass or fibrosis was detected (Figure 1). In contrast to the above-mentioned patterns of fibrotic changes, in which there was a clear correlation with the initial tumour size and features at MRI before chemoradiotherapy, no such correlation was found in rectal tumours that fully disappeared after CRT. Some of these patients had initially shown relatively large, bulky tumours, whereas others had shown small polypoid tumours (Table 3).

\section{MRI findings at long-term follow-up}

Interestingly, in the 14 patients in whom a residual area of fibrosis was detected after CRT, the aspect of the fibrosis remained largely unaffected during long-term follow-up. No changes were observed regarding the shape, delineation or hypointense signal intensity of the fibrotic areas during repeated follow-up MRIs and a further volume decrease, if any, was only very subtle. In the patients in whom 
the tumour vanished at MRI and the rectal wall normalised, this normal wall persisted on consecutive MRIs at longer follow up and no long term evolution into fibrosis was observed (Table 3). The longest follow-up at the time of writing was 60 months.

\section{Edema}

In the first months after completion of chemoradiation treatment, a small group (5/19; 26\%) of the patients showed an edematous rectal wall, visible as a characteristic three layered pattern of dark mucosa, bright submucosa and dark muscular wall. In all patients this edematous thickening gradually decreased during the remaining follow-up period (Figure 4; Table 2).
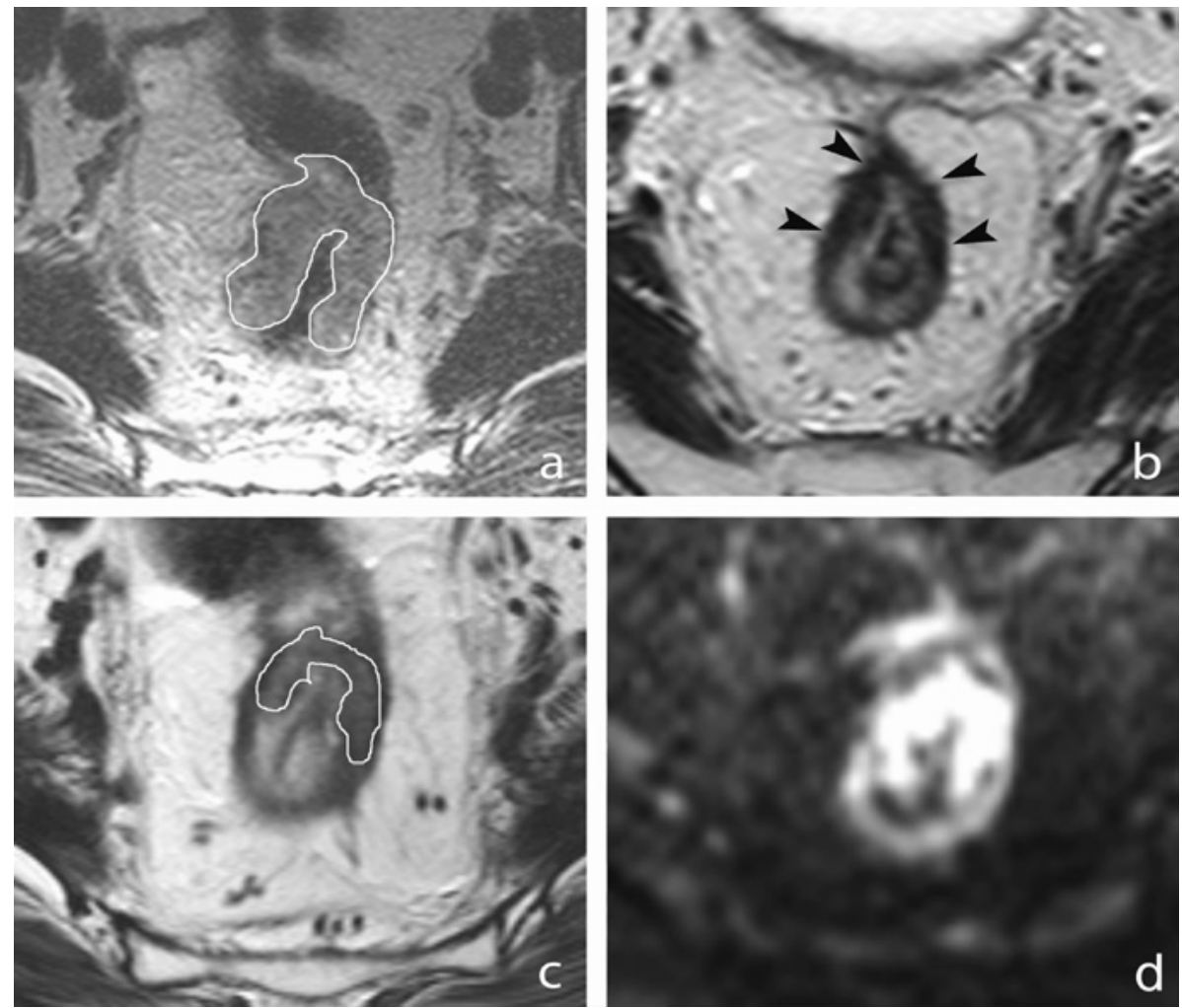

Figure 7. Local tumour recurrence during follow-up. Axial MR images of a patient who was nonoperatively managed but developed a local recurrence during follow up. The pre-CRT MRI (a) shows a grey tumour in the proximal rectum. After chemoradiation, the tumour shrunk and the rectal wall turned fibrotic (b). After two years of follow up, a grey mass was visible in the fibrotic tumour bed (c) suggestive of a recurrent tumour. On the corresponding diffusion-weighted MR image (d), the recurrent tumour could easily be recognised as an area of markedly high signal intensity. The presence of recurrent adenocarcinoma was proven by tumour biopsy. 


\section{Persistent complete response versus recurrent tumour}

One patient from the study group developed a small endoluminal recurrence after 22 months of follow-up (Table 2). Endorectal ultrasound indicated a T1 tumour and the patient underwent a salvage procedure with transanal endoscopic microsurgery. At present, he is disease-free, but with only a follow-up of 2 months after the procedure. For illustrative purposes, we report on two additional patients outside the study group in whom the tumour locally recurred after 17 and 24 months of follow-up. These two patients were not part of the study group because they presented with synchronous distant (liver) metastases at their initial diagnosis. They both received a long-course of neoadjuvant chemoradiation after which they were curatively treated for their liver metastases. On the restaging MRI performed 6-8 weeks after completion of CRT, a dark area of fibrosis was visible in the irradiated tumour bed and no residual tumour could be detected. Both patients chose not to undergo total mesorectal excision surgery. During their long-term follow-up, the volume of the previously fibrotic tumour bed increased and the signal intensity changed from black (indicating fibrosis) to grey (indicating tumour) on the T2-weighted MR images. In addition, areas of high signal intensity, indicative of recurrent tumour, developed on the diffusion-weighted images (Figure 7). In the patient with the small local recurrence in the wait-and-see study group, the T2weighted MR images did not yet reveal any significant changes in the morphology of the rectal wall. The diffusion-weighted MRI, however, showed a small area of high signal intensity, compatible with a tumour recurrence.

\section{Discussion}

In many countries, MRI is part of the standard workup for the staging of rectal cancer at primary diagnosis. ${ }^{1}$ In recent years, patients with locally advanced rectal cancer who receive preoperative chemo and/or radiation therapy have shown phenomenal response with downstaging and downsizing of the tumour. This opens the debate regarding whether a resection is still necessary in all patients. It is in this setting that a restaging MRI after CRT could be helpful. Until now, the main aim of a restaging MRI has been to provide the surgeon with a roadmap to determine his resection plane and to decide whether a less extensive resection can be performed in tumours that have regressed from the mesorectal fascia. ${ }^{16}$ Given the current trend toward minimally invasive treatments for the good responders after CRT, a more precise imaging assessment of response is mandatory to improve the selection of these patients and the response monitoring at follow-up. Although the selection of patients is generally based on clinical examination and endoscopy with bi- 
opsy, imaging could help in increasing the confidence for appropriate selection of the right patients. In our hospital, the imaging method used for restaging is MRI. In addition to a restaging MRI shortly after CRT, the current study protocol in which patients underwent a wait-and-see approach with frequent follow-up ${ }^{5}$ allowed us to also monitor the evolution of MR features during long-term follow-up. We believe that all multidisciplinary team members, including colorectal surgeons, need to understand the MR images and the patterns of response.

This study aimed at describing and illustrating the evolution of the tumour bed on consecutive MRIs in a selected group of patients with rectal cancer with a clinical complete tumour response after CRT who were managed with a wait-andsee policy. The MRI of the rectum in these patients at the moment of their selection (6-8 wk after completion of CRT) and the period thereafter showed interesting evolution patterns. The MR morphology of the complete tumour responders 6 to 8 weeks after CRT was typically that of either a dark fibrotic wall thickening or, in a smaller number of patients, a normalised rectal wall. Given the difficulties of MRI in the interpretation of fibrosis, the feature of a normalised wall on post-CRT MR images will likely be the criterion with the highest positive predictive value for identifying a complete response by imaging. In our study, there was no clear correlation between the initial tumour morphology before treatment and the chance of a completely normalised rectal wall after CRT. It may be that, in some tumours, the biological tumour profile rather than the morphological characteristics determines the way the tumour will react to CRT. In contrast, the morphological pattern of the fibrotic areas did follow that of the primary tumour before the onset of treatment. These patterns could be categorised as "full-thickness," "minimal," or "irregular" fibrosis and remained consistent over the years. When the tumour had completely disappeared and the post-CRT MRI showed a normalised rectal wall, this normal wall persisted over the years. When, during the first months after CRT, the wall showed an edematous thickening, this edema gradually disappeared during longer follow-up. All together, if the morphology (normalised wall or fibrosis) as identified on the first restaging MRI remained consistent during long-term follow-up, this could be considered a sign of a persistent complete response. Although our study was not designed to systematically compare patients with a complete response with patients with recurrent tumour, we did find that in patients with recurrent disease, areas of intermediate signal intensity began to develop within the fibrotic scar bed on the T2-weighted images. This is in concordance with previous reports that showed that the development of areas of intermediate signal intensity during follow-up was predictive for the presence of a local tumour recurrence. ${ }^{17,18}$ The small recurrence in the wait-and-see patient was noted on diffusion-weighted MRI as a newly developed area of high signal intensity, whereas the T2-weighted images did 
not yet reveal any significant changes in morphology. It has previously been suggested that diffusion-weighted MRI may be superior to conventional MRI in the detection of (small) malignant tumours, in particular, within areas of postradiation fibrosis. ${ }^{14,15,19}$ Diffusion-weighted MRI could thus be a valuable adjunct to standard imaging in the detection of recurrent tumour during the nonoperative follow-up of patients with rectal cancer, although the true value of diffusion-weighted imaging in this particular clinical setting will need to be addressed by further studies.

Our study design has some limitations. First, a "definite" histological diagnosis of a complete response could not be obtained because none of the patients underwent surgical resection. In the clinical setting of a wait-and-see approach, the usual standard of histology is inherently not available, and an extensive clinical follow-up as applied in our study may serve as a surrogate reference standard. Ideally, the follow-up period should have been at least 2 years for all patients, because most tumour recurrences are likely to occur within the first 2 years after treatment. ${ }^{20}$ Our results should therefore be considered preliminary, and larger patient groups with longer follow-up are required to draw more definite conclusions. Second, the aim of our study was to study the evolution of the MR morphology of patients with a known complete response and not to study the potential of MRI in the initial identification and selection of complete responders, which has been the subject of previous studies, including a study from our center. ${ }^{14,15}$

In conclusion, four MR patterns of a persistent complete response of rectal cancer after chemoradiation are identified. These MR features can serve as a reference for the follow-up of patients treated with a 'wait and see policy'. 


\section{References}

1. Glimelius B, Oliveira J. Rectal cancer: ESMO clinical recommendations for diagnosis, treatment and follow-up. Ann Oncol. 2009; 20 (Suppl 4):54-56.

2. Habr-Gama A, Perez RO, Proscurshim I, et al. Patterns of failure and survival for nonoperative treatment of stage c0 distal rectal cancer following neoadjuvant chemoradiation therapy. J Gastrointest Surg 2006; 10:1319-1328; discussion 1328-1319.

3. Lezoche $\mathrm{G}$, Baldarelli M, Guerrieri M, et al. A prospective randomized study with a 5-year minimum follow-up evaluation of transanal endoscopic microsurgery versus laparoscopic total mesorectal excision after neoadjuvant therapy. Surg Endosc 2008; 22:352-358.

4. Borschitz $T$, Wachtlin D, Mohler M, Schmidberger $H$, Junginger T. Neoadjuvant chemoradiation and local excision for T2-3 rectal cancer. Ann Surg Oncol 2008; 15:712-720.

5. Maas M, Lambregts DMJ, Van Dam RM, et al. MR-based 'wait-and-see' policy in clinical complete responders to chemoradiation in rectal cancer: A promising alternative. Ann Oncol 2010; 21 (Suppl 1) :i54 [abstract].

6. Yu SK, Brown G, Heald RJ, et al. Deferral of rectal cancer surgery following a continued response to preoperative chemoradiotherapy (Watch and Wait) study: a phase II multicenter study in the United Kingdom. J Clin Oncol 2011; 29 (Suppl 4):abstr 489 [abstract].

7. Carlomagno C, Pepe S, D'Armiento FP, et al. Predictive factors of complete response to neoadjuvant chemoradiotherapy in patients with rectal cancer. Oncology; 78:369-375.

8. Kim SH, Lee JY, Lee JM, Han JK, Choi BI. Apparent diffusion coefficient for evaluating tumor response to neoadjuvant chemoradiation therapy for locally advanced rectal cancer. Eur Radiol 2011; $21: 987$ 995

9. Kim DJ, Kim JH, Lim JS, et al. Restaging of Rectal Cancer with MR Imaging after Concurrent Chemotherapy and Radiation Therapy. Radiographics 2010; 30:503-516.

10. Barbaro $B$, Vitale $R$, Leccisotti $L$, et al. Restaging locally advanced rectal cancer with MR imaging after chemoradiation therapy. Radiographics 2010; 30:699-716.

11. Habr-Gama A, Perez RO, Wynn G, Marks J, Kessler H, Gama-Rodrigues J. Complete clinical response after neoadjuvant chemoradiation therapy for distal rectal cancer: characterization of clinical and endoscopic findings for standardization. Dis Colon Rectum 2010; 53:1692-1698.

12. Janssen $M H$, Ollers MC, Riedl RG, et al. Accurate Prediction of Pathological Rectal Tumor Response after Two Weeks of Preoperative Radiochemotherapy Using (18)F-Fluorodeoxyglucose-Positron Emission Tomography-Computed Tomography Imaging. Int J Radiat Oncol Biol Phys 2010;77:392399.

13. van Stiphout RG, Lammering G, Buijsen J, et al. Development and external validation of a predictive model for pathological complete response of rectal cancer patients including sequential PET-CT imaging. Radiother Oncol 2011; 98:126-133.

14. Kim SH, Lee JM, Hong SH, Kim GH, Lee JY, Han JK, Choi BI. Locally advanced rectal cancer: added value of diffusion-weighted MR imaging in the evaluation of tumor response to neoadjuvant chemoand radiation therapy. Radiology 2009; 253:116-125

15 Lambregts DM, Vandecaveye V, Barbaro B, et al. Diffusion-weighted MRI for selection of complete responders after chemoradiation for locally advanced rectal cancer: a multicenter study. Ann Surg Oncol 2011; 18:2224-2231.

16. Vliegen RF, Beets GL, Lammering G, et al. Mesorectal fascia invasion after neoadjuvant chemotherapy and radiation therapy for locally advanced rectal cancer: accuracy of MR imaging for prediction. Radiology 2008; 246:454-462.

17. Blomqvist L, Holm T, Göranson $H$, Jacobsson $H$, Ohlsén $H$, Larsson SA. MR imaging, CT and CEA scintigraphy in the diagnosis of local recurrence of rectal carcinoma. Acta Radiol 1996; 37:779-784

18. Lambregts DM, Cappendijk VC, Maas M, Beets GL, Beets-Tan RGH. Value of MRI and diffusionweighted MRI for the diagnosis of locally recurrent cancer. Eur Radiol 2011; 21:1250-1258 
19. Nishie A, Stolpen AH, Obuchi M, Kuehn DM, Dagit A, Andresen K. Evaluation of locally recurrent pelvic malignancy: performance of T2- and diffusion-weighted MRI with image fusion. J Magn Reson Imaging 2008; 28:705-713

20. Habr-Gama A, Perez RO, Nadalin W, et al. Operative versus nonoperative treatment for stage 0 distal rectal cancer following chemoradiation therapy: long-term results. Ann Surg 2004; 240:711-717; discussion 717-718 


\section{VALUE OF MRI AND DIFFUSION-WEIGHTED MRI FOR THE DIAGNOSIS OF LOCALLY RECURRENT RECTAL CANCER}

\section{CHAPTER}

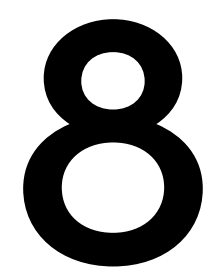

DMJ Lambregts VC Cappendijk M Maas GL Beets RGH Beets-Tan

Published in: European Radiology 2011; 21(6):1250-1258 


\section{Abstract}

\section{Aim}

To evaluate the accuracy of standard MRI, diffusion-weighted MRI (DWI) and fusion images for the diagnosis of locally recurrent rectal cancer in patients with a clinical suspicion of recurrence.

\section{Materials \& Methods}

Forty-two patients with a clinical suspicion of recurrence underwent 1.5-T MRI consisting of standard T2-weighted FSE (3 planes) and an axial DWI (b0,500,1000). Two readers $(R 1, R 2)$ independently scored the likelihood of recurrence; $(a)$ on standard MRI, (b) on standard MRI+DWI, and (c) on T2-weighted+DWI fusion images.

\section{Results}

19/42 patients had a local recurrence. R1 achieved an area under the ROC-curve (AUC) of 0.99 , sensitivity $100 \%$ and specificity $83 \%$ on standard MRI versus 0.98 , $100 \%$ and $91 \%$ after addition of DWI $(P=0.78)$. For R2 these figures were $0.87,84 \%$ and $74 \%$ on standard $\mathrm{MRI}$ and $0.91,89 \%$ and $83 \%$ with DWI $(P=0.09)$. Fusion images did not significantly improve the performance. Interobserver agreement was $\kappa 0.69$ for standard MRI, $\kappa 0.82$ for standard MRI+DWI and $\kappa 0.84$ for the fusion images.

\section{Conclusions}

MRI is accurate for the diagnosis of locally recurrent rectal cancer in patients with a clinical suspicion of recurrence. Addition of DWI does not significantly improve its performance. However, with DWI specificity and interobserver agreement increase. Fusion images do not improve accuracy. 


\section{Introduction}

Around $35 \%$ of colorectal cancer patients who undergo curative surgical treatment will develop recurrent disease, the majority of which occurring during the first three years after surgery. ${ }^{1}$ Relapse mainly occurs in the liver (20\%) and lungs (5-10\%), while approximately $5-15 \%$ of patients will develop a locoregional recurrence. ${ }^{2}$ These local recurrences are associated with significant morbidity and impaired quality of life. Whereas locally recurrent rectal cancer has long been regarded as a rarely curable disease, patients are now increasingly being considered for curative salvage surgery, often combined with (neo)adjuvant chemo and/or radiation treatment. The chances of cure after resection are highest when all resection margins are microscopically free of tumour. ${ }^{3}$ To increase the chance of cure it is therefore crucial to diagnose local recurrences when still in an early stage. At present, it is not clear whether imaging is beneficial during the surveillance of patients after rectal cancer surgery and trials to establish the role of imaging are ongoing. ${ }^{4-7}$ If, however, during surveillance a patient is suspected of a local recurrence based on clinical symptoms and/or rising carcinoembryonic antigen (CEA) levels, CT is the first investigation of choice to confirm or rule out the presence of a local or distant relapse. ${ }^{4,7}$ A pelvic mass that enlarges on consecutive postoperative CT studies is highly suspicious for a local recurrence, although the diagnosis is not always easy to make. ${ }^{9,10}$ Often the CT findings are equivocal and in those cases ${ }^{18} \mathrm{~F}$-Fluorodeoxygenase Positron Emission Tomography (FDG-PET) or combined PET/CT have proven beneficial in identifying local tumour regrowth. ${ }^{10,11}$ False positive FDG-PET findings may, however, occur in areas of post operative inflammatory scar tissue, in the case of displaced small bowel loops and because of the proximity of the urinary bladder. Moreover, FDG-PET lacks accuracy in the evaluation of a recurrence of the mucinous tumour type and is less useful to evaluate tumour resectability due to its insufficient spatial resolution. ${ }^{11,12} \mathrm{MRI}$ on the other hand has proven valuable for assessing the resectability of diagnosed local recurrence of rectal cancer ${ }^{13}$, but its role in the detection of recurrent tumour is not well established. The main difficulty with MRI is the identification of a small growing tumour in an area of fibrotic scar tissue. ${ }^{14-16}$ Diffusion-weighted MR imaging (DWI) is a promising technique for the detection of small tumour volumes and its benefit has also been shown in pelvic tumours. ${ }^{17-19}$ The role of DWI in the detection of locally recurrent rectal cancer has, however, not been investigated yet. As an addition to standard anatomical MRI, DWI could be useful to increase the diagnostic performance for detection of locoregional tumour recurrences. ${ }^{20}$ Furthermore, previous studies have suggested that image fusion of anatomical MRI and DWI can contribute to a better detection of both primary malignancies and recurrent disease, though again its role in rectal cancer is not clear. ${ }^{20,21}$ 
Table 1. Baseline characteristics of study patients

\begin{tabular}{|c|c|c|c|}
\hline \multirow[b]{2}{*}{ Variable } & \multicolumn{3}{|c|}{$\mathrm{N}^{\circ}$ Patients } \\
\hline & $\begin{array}{l}\text { Recurrence } \\
\quad(n=19)\end{array}$ & $\begin{array}{l}\text { No Recurrence } \\
\qquad(n=23)\end{array}$ & $\begin{array}{l}\text { Total } \\
(n=42)\end{array}$ \\
\hline \multicolumn{4}{|l|}{ Sex } \\
\hline Male & 13 & 13 & 26 \\
\hline Female & 6 & 10 & 16 \\
\hline Median age & 68 (range $35-87$ ) & 64 (range 22-81) & 66 (range 22-87) \\
\hline \multicolumn{4}{|l|}{ Treatment of primary tumour } \\
\hline Surgery & 6 & 3 & 9 \\
\hline $5 x 5$ Gy + surgery & 2 & 5 & 7 \\
\hline $\begin{array}{l}\text { Neoadjuvant and/or adjuvant } \\
\text { chemoradiation + surgery }\end{array}$ & 11 & 15 & 26 \\
\hline \multicolumn{4}{|l|}{ Type of primary surgery } \\
\hline Total mesorectal excision & 14 & 22 & 36 \\
\hline $\begin{array}{l}\text { Local excision (transanal } \\
\text { endoscopic microsurgery) }\end{array}$ & 5 & 1 & 6 \\
\hline \multicolumn{4}{|l|}{ Radicality of primary resection } \\
\hline RO & 14 & 19 & 33 \\
\hline $\mathrm{R} 1$ & 3 & 4 & 7 \\
\hline $\mathrm{R} 2$ & 2 & 0 & 2 \\
\hline \multicolumn{4}{|l|}{ Synchronous metastases } \\
\hline Liver & 5 & 4 & 9 \\
\hline Lung & 1 & 1 & 2 \\
\hline Other & 0 & 0 & 0 \\
\hline \multicolumn{4}{|l|}{ Metachronous metastases } \\
\hline Liver & 4 & 4 & 8 \\
\hline Lung & 5 & 5 & 10 \\
\hline Other & 4 & 2 & 6 \\
\hline Median CEA at time of MRI $(\mu \mathrm{g} / \mathrm{l})$ & 8 (range 1-2154) & 3 (range $0,5-55$ ) & 4 (range $0,5-2154$ ) \\
\hline Previous recurrence & 4 & 2 & 6 \\
\hline
\end{tabular}


The aim of this study therefore is to evaluate the accuracy of standard pelvic MRI for the diagnosis of locoregional tumour recurrence in patients with a clinical suspicion of a recurrence after curative rectal cancer surgery and to evaluate whether the addition of DWI and/or image fusion of standard MRI and DWI improves its performance.

\section{Materials and Methods}

\section{Patients}

This study retrospectively evaluated 42 consecutive patients who underwent MR imaging for the evaluation of the presence of a locally recurrent rectal cancer between June 2007 and April 2010. Because of the retrospective nature of the study, informed consent was waived. The baseline patient characteristics are given in Table 1. Twenty-six patients were male and 16 were female. Median age was 66 years (range 22-87). All patients had previously undergone curative-intent surgical treatment for primary rectal cancer. Patients were referred for MRI when during follow-up there was a clinical suspicion for a locoregional tumour recurrence based on symptoms (pain, faecal blood loss, changed bowel habits), clinical examination and/or increased serum CEA and/or the presence of metachronous distant tumour lesions. Eleven patients first underwent a CT examination, before being referred for MRI. Nineteen patients were diagnosed with a locally recurrent rectal tumour, while in 23 patients no evidence of recurrence was found. The locations of recurrence were as follows: anastomotic $(n=2)$, presacral $(n=3)$, lateral pelvic nodes $(n=3)$, pelvic floor $(n=3)$, local excision scar $(n=5)$, rectal lumen $(n=1)$, peri-prostatic ( $n=1)$, and gluteus muscle $(n=1)$. The median time interval between surgical treatment of the primary rectal tumour and follow-up MRI was 40 months (range 3-320). Median follow-up between the MRI for local recurrence detection and present was 20 months (range 4-42).

\section{MR imaging}

Patients did not receive bowel preparation or spasmolytics. MR imaging was performed at 1.5-T (Intera; Philips Medical Systems, Best, The Netherlands). For signal reception, a phased-array body coil was used. The standard rectal MRI protocol consisted of 2D T2-weighted (T2W) fast spin-echo (FSE) sequences in three planes; sagittal, axial and coronal (TR/TE $8456 / 130 \mathrm{msec}, 90^{\circ}$ flip angle, 25 echotrain length, $6 \mathrm{NSA}, 0.78 \times 1.14 \times 3.00 \mathrm{~mm}$ acquisition voxel size, 30 slices, 6.03 minutes acquisition time). Since 2006, a DWI sequence had been implemented as part of the 
standard rectal MRI protocol. The DWI sequence was an axial diffusion-weighted sequence with background body signal suppression $\left(D^{2} I_{B S}{ }^{22}\right.$, b-values 0,500,1000 $\mathrm{s} / \mathrm{mm}^{2}$, TR/TE 4829/70 msec, EPI factor 53, $4 \mathrm{NSA}, 2.50 \times 3.11 \times 5.00 \mathrm{~mm}$ acquisition voxel size, 50 slices, 10.37 minutes acquisition time). The axial T2-weighted and DWI sequences were obtained in identical planes. These were routinely angled perpendicular to the rectal lumen. When no remaining rectal lumen was present (after abdominoperineal or extended resection) or in case of a pelvic mass located outside the rectal lumen, the axial sequences were angled perpendicular to the interface between the local area of suspicion and the closest structure or organ. ADC maps including all three $b$-values were automatically generated in greyscale at the operating system.

\section{Image fusion of T2-weighted MRI and DWI}

MR images were exported in DICOM format and transferred to a Macintosh computer (iMac, Apple, Cupertino, CA, USA). Fusion images of the axial T2-weighted and b1000 diffusion-weighted images were generated using OsiriX Medical Imaging Software, an open-source post-processing software tool. ${ }^{23}$ The field of view and slice level of the diffusion images were automatically adjusted to exactly match the T2-weighted MRI. The DWI images were then converted to a red-to-yellow colour scale and overlaid on the T2-weighted images.

\section{Image evaluation}

The MR images were independently analysed by two readers from an expert referral center: a pelvic MR expert with 13 years expertise in reading MRI of rectal cancer (RGHBT) and a junior GI radiologist with 3 years specific expertise in pelvic MRI (VCC). The readers were aware of the treatment, histological stage and tumour type of the primary rectal cancer, but were blinded to each other's scoring results and to whether or not previous CT imaging for the recurrence was present. They were also blinded to the results from endoscopy, other imaging, surgery and histopathology. This study design was chosen to reproduce the clinical standard of practice, where radiologists are exposed to a patient's history of the primary tumour. Image evaluation consisted of three separate reading protocols conducted within the same reading session: (a) the readers first evaluated the T2-weighted FSE images only (further referred to as 'standard MRI'), (b) immediately thereafter the scoring was repeated after the b1000 diffusion images were added to the standard MRI (further referred to as 'standard MRI + DWI'). The low b-value diffusion images and the ADC map were also at the readers' disposal. Finally (c) the fusion images of T2-weighted MRI + DWI were assessed. Patients were evaluated in random order. 
The readers scored the likelihood of a local tumour recurrence using a 5-point confidence level scale $(0=$ definitely absent, 1 = probably absent, 2 = possibly absent/present, 3 = probably present, 4 = definitely present). On T2-weighted MRI, focal areas showing a mass effect, a signal intensity isointense to muscle and/or a nodular or infiltrative border were considered highly suspicious for tumour recurrence. On the high b-value (b1000) DWI, focal areas of high signal intensity, corresponding with possible tumour locations on standard MRI were considered a criterion for tumour recurrence (Figure 1). On the fusion images focal areas of hyperintensity (yellow), corresponding with possible tumour on T2-weighted MRI were considered suspicious for recurrent tumour. When a recurrent tumour was suspected, the largest tumour diameter was measured on T2-weighted MRI.

\section{Standard of reference}

The presence of a local recurrence $(n=19)$ was histologically confirmed in 16 patients (surgical resection in 11 and positive biopsy of the suspected tumour area in 5 patients). In three patients the diagnosis of a local recurrence was made based on concordant positive FDG-PET/CT findings (i.e. positive FDG uptake at the exact location suspected for tumour on MRI) as well as follow-up MRI and/or FDG$\mathrm{PET} / \mathrm{CT}$ examinations. Furthermore, an inguinal lymph node metastasis was confirmed by biopsy in one patient. These three patients did not undergo surgical treatment of their recurrence, because of unresectable disease. The absence of a local recurrence $(n=23$ ) was confirmed by a combination of the following: no abnormal findings on corresponding FDG/PET-CT $(n=8)$, no abnormal findings on colonoscopy and/or negative biopsy results $(n=4)$ and/or no signs of tumour recurrence on consecutive imaging examinations (CT, MRI and/or FDG-PET/CT) during a follow-up period of at least one year $(n=22)$. Median follow-up time for the patients without recurrence was 23 months (range 14-41).

\section{Statistical analyses}

Statistical analyses were performed using the Statistical Package for the Social Sciences (SPSS, version 16.0, Inc., Chicago, IL). Receiver operator characteristic (ROC) curve analyses were performed to evaluate the diagnostic performance for (a) standard MRI only, (b) standard MRI + DWI and (c) fused T2-weighted + DWI images. Corresponding areas under the ROC-curve (AUC), sensitivities, specificities, positive predictive values (PPV) and negative predictive values (NPV) with $95 \%$ confidence intervals $(\mathrm{Cl})$ were calculated. For these analyses it had been decided at the start of the study to dichotomise the confidence level scores between 1 (probably absent) and 2 (possibly absent/present). Differences in diagnostic 
performance between the 3 reading strategies were analysed by comparing the ROC-curves according to the method described by DeLong et al. ${ }^{24}$ Weighted kappavalues with quadratic kappa weighting (0.00-0.20 poor, 0.21-0.40 fair, 0.41-0.60 moderate, $0.61-0.80$ good and 0.81-1.00 excellent interobserver agreement) were calculated to determine interobserver agreement between the two readers. ${ }^{25}$ $P$-values $<0.05$ were considered statistically significant.

\section{Results}

Diagnostic predictive values including $95 \%$ confidence intervals are provided in Table 2 for the three reading methods. The ROC-curves are displayed in Figure 2.

Table 2. Diagnostic performance for identification of a local tumour recurrence

\begin{tabular}{|c|c|c|c|c|c|c|}
\hline \multirow{2}{*}{ Parameter } & \multicolumn{3}{|c|}{ Reader 1} & \multicolumn{3}{|c|}{ Reader 2} \\
\hline & $\begin{array}{c}\text { Standard } \\
\text { MRI }\end{array}$ & $\begin{array}{l}\text { Standard } \\
\text { MRI + DWI }\end{array}$ & Fusion & $\begin{array}{c}\text { Standard } \\
\text { MRI }\end{array}$ & $\begin{array}{l}\text { Standard } \\
\text { MRI + DWI }\end{array}$ & Fusion \\
\hline Sensitivity & $100(19 / 19)$ & $100(19 / 19)$ & $100(19 / 19)$ & $84(16 / 19)$ & 89 (17/19) & $84(16 / 19)$ \\
\hline $95 \% \mathrm{Cl}$ & $88-100$ & $89-100$ & $89-100$ & $68-94$ & $75-97$ & $69-93$ \\
\hline Specificity & $83(19 / 23)$ & $91(21 / 23)$ & $91(21 / 23)$ & $74(17 / 23)$ & $83(19 / 23)$ & $83(19 / 23)$ \\
\hline $95 \% \mathrm{Cl}$ & $73-83$ & $82-91$ & $82-91$ & $61-82$ & $70-89$ & $70-90$ \\
\hline PPV & $83(19 / 23)$ & $90(19 / 21)$ & $90(19 / 21)$ & $71(15 / 21)$ & $80(16 / 20)$ & $79(15 / 19)$ \\
\hline $95 \% \mathrm{Cl}$ & $73-83$ & $80-91$ & $80-91$ & $59-81$ & $68-88$ & $65-89$ \\
\hline NPV & 100 (19/19) & $100(21 / 21)$ & $100(21 / 21)$ & $85(17 / 20)$ & $90(19 / 21)$ & $86(19 / 22)$ \\
\hline $95 \% \mathrm{Cl}$ & $88-100$ & $90-100$ & $90-100$ & $70-94$ & $77-97$ & $73-94$ \\
\hline Accuracy & $90(38 / 42)$ & $95(40 / 42)$ & $95(40 / 42)$ & $79(33 / 42)$ & $86(36 / 42)$ & $83(35 / 42)$ \\
\hline $95 \% \mathrm{Cl}$ & $79-91$ & $85-95$ & $85-95$ & $65-87$ & $72-92$ & $70-92$ \\
\hline AUC & 0.99 & 0.98 & 0.98 & 0.87 & 0.91 & 0.91 \\
\hline $95 \% \mathrm{Cl}$ & $0.96-1.00$ & $0.94-1.00$ & $0.93-1.00$ & $0.75-0.98$ & $0.82-1.00$ & $0.82-1.00$ \\
\hline
\end{tabular}

PPV = positive predictive value, NPV = negative predictive value, AUC = area under the ROC curve, $95 \%$ $\mathrm{Cl}=95 \%$ confidence interval. Numbers are percentages, absolute numbers are given in parentheses. Reader 1 is a senior GI radiologist with 13 years specific pelvic MR expertise. Reader 2 is a junior GI radiologist with 3 years pelvic MR expertise. 


\section{Standard MRI}

When using only standard MRI, reader 1 achieved an AUC of 0.99 , sensitivity $100 \%$ and specificity $83 \%$. For reader 2 these figures were $0.87,84 \%$ and $74 \%$.

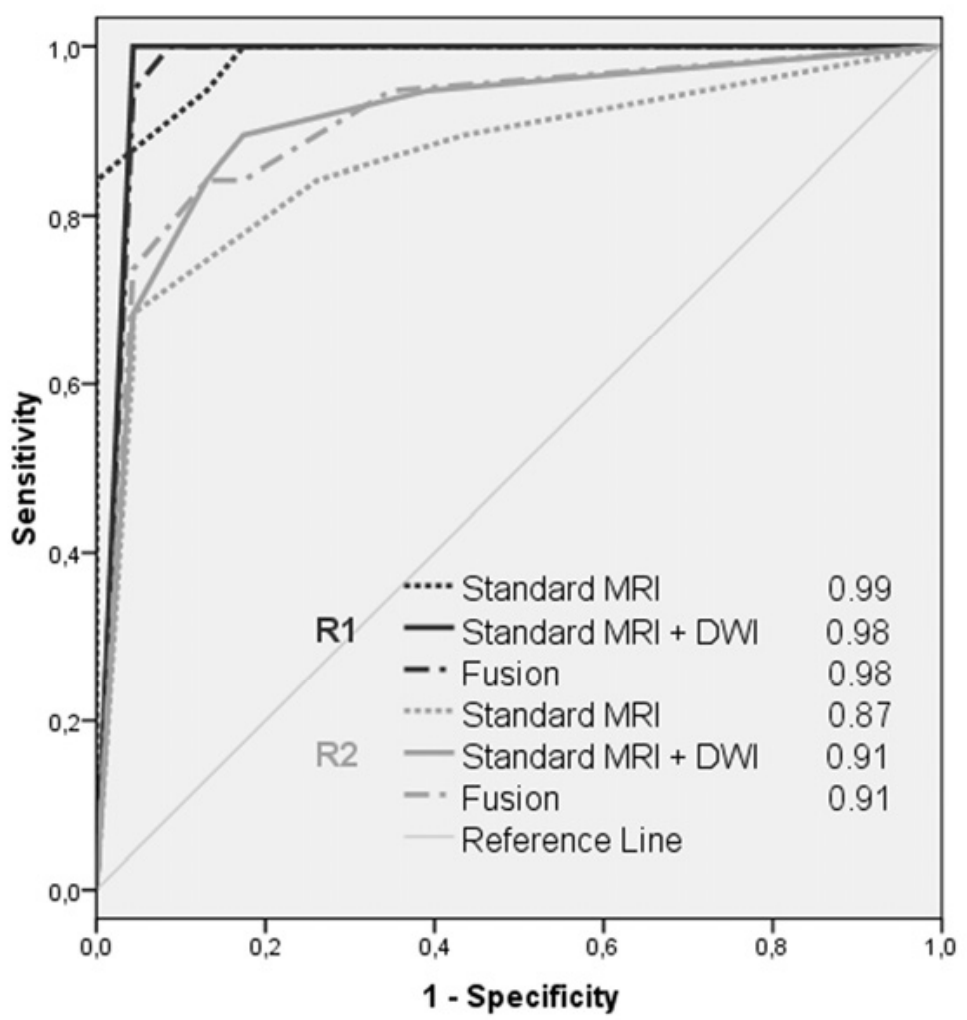

Figure 2. Receiver operator characteristics curves and areas under the ROC-curve (AUC) for reader 1 (R1) and reader 2 (R2) for detection of locally recurrent rectal cancer by using (a) standard MRI, (b) standard $\mathrm{MRI}+\mathrm{DWI}$, and (c) T2-weighted MRI + DWI fusion images, respectively. There were no significant differences in AUC between the three reading designs for either of the two readers.

\section{Added value of DWI and fusion images}

After addition of DWI, reader 1 achieved an AUC of 0.98 , sensitivity $100 \%$ and specificity $91 \%$. Compared to standard MRI (AUC 0.99), the difference in AUC was not significant $(P=0.78)$. For reader 2 , AUC improved from 0.87 on standard MRI to 0.91 after addition of DWI $(P=0.09)$. He achieved a sensitivity of $89 \%$ and specificity of $83 \%$. The added value of DWI is illustrated in Figure 3 , in which a false positive 
finding on T2-weighted MRI was corrected after addition of DWI. The addition of fusion images did not improve the diagnostic performance as compared to standard $\mathrm{MRI}+\mathrm{DWI}(P=0.41$ for reader 1 and $P=1.00$ for reader 2$)$.
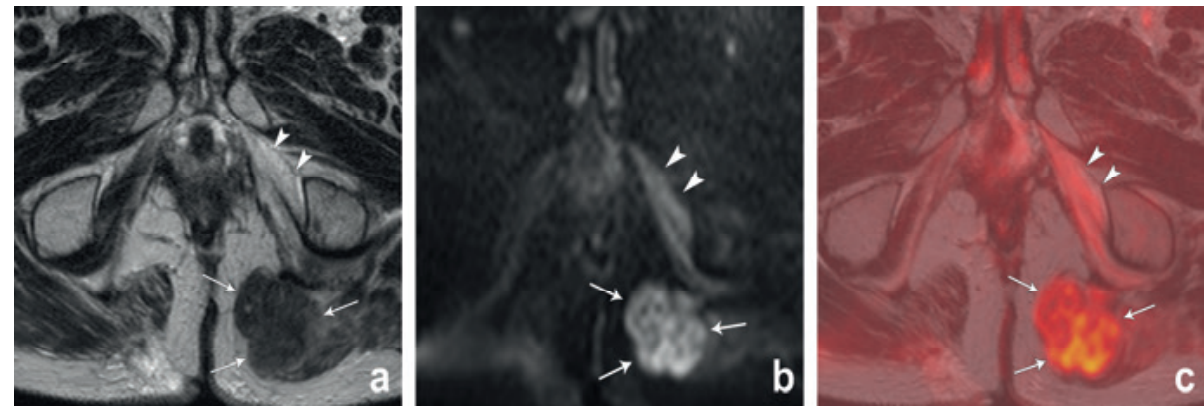

Figure 1. T2-weighted, b1000 diffusion-weighted and T2W + DWI fusion images of a male patient with a local tumour recurrence situated in the left gluteus muscle (arrows). On T2-weighted MRI (a), the signal of the tumour is isointense compared to the muscles. On the DWI (b) and fusion images (c) the tumour is visible as a lesion with high signal intensity, compared to the suppressed signal of the surrounding structures. Note the necrotic changes in the left internal obturator muscle (arrowheads), resulting in slightly increased signal intensity on the DWI and fusion images.
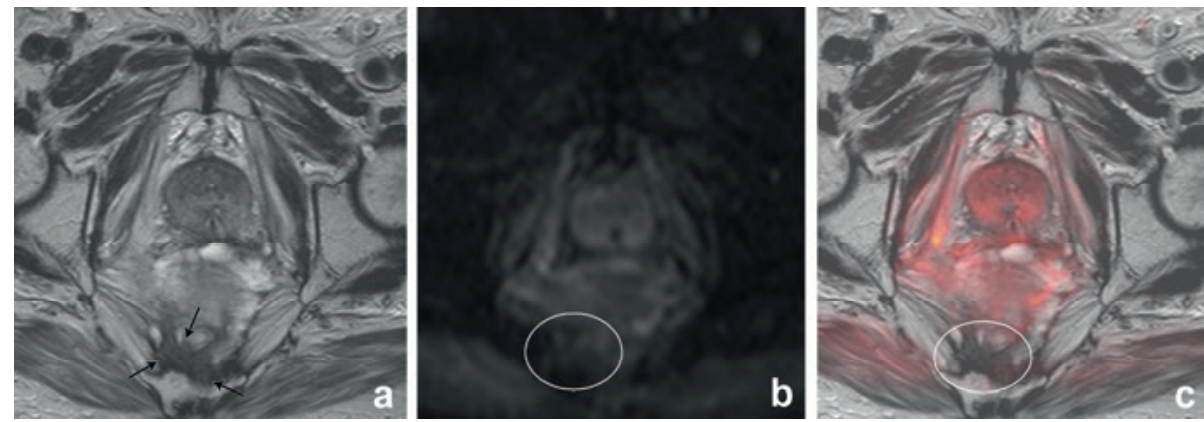

Figure 3. Axial T2-weighted, b1000 diffusion-weighted and T2W + DWI fusion images of a male patient who had previously undergone a low anterior resection. On T2-weighted MRI (a) there is an area of intermediate signal intensity (arrows) that was erroneously interpreted as a tumour recurrence by the first reader. On the DWI (b) and fusion images (c), there is no focal high signal intensity in this area (circle) and the reader adjusted his score. Follow-up imaging revealed no tumour recurrence.

\section{Interobserver agreement}

Interobserver agreement for standard MRI was good ( $\kappa$ 0.69). After the addition of DWI, agreement was excellent $(\kappa 0.82)$. Agreement for the T2W $+\mathrm{DWI}$ fusion images was excellent ( $\kappa$ 0.84). 


\section{Lesion size}

In the 19 patients with recurrence, median lesion size (largest tumour diameter) was $37 \mathrm{~mm}$ (range 13-81 mm). Seven patients had a 'small' recurrence $(<20 \mathrm{~mm})$. Reader 1 detected all 7 small recurrences, both with standard MRI and after addition of DWI. Reader 2 missed one small recurrence on standard MRI, but detected all 7 after addition of DWI (Figure 4).

Eleven patients had undergone CT imaging before MRI. In Table 3 and Figure 5 the discrepancies between the CT findings and MRI scorings are illustrated.
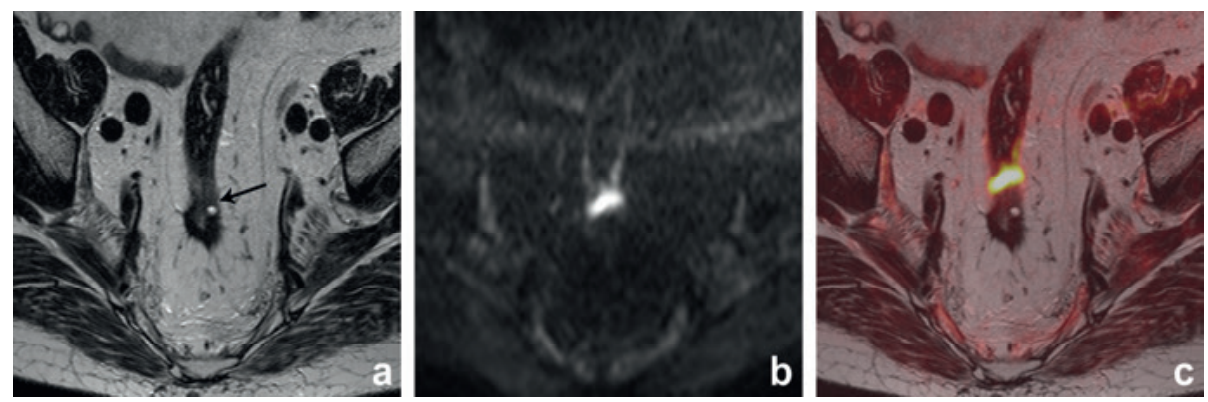

Figure 4. Axial T2-weighted, b1000 diffusion-weighted and T2W + DWI fusion images of a male patient who had previously undergone transanal endoscopic microsurgery. On T2-weighted MRI (a) there is a small area of intermediate signal intensity (arrow), that was overlooked by reader 2. On the DWI (b) and fusion images (c), there is a clear focal area of high signal intensity and the reader now identified the recurrence, which was later surgically removed and histologically confirmed.
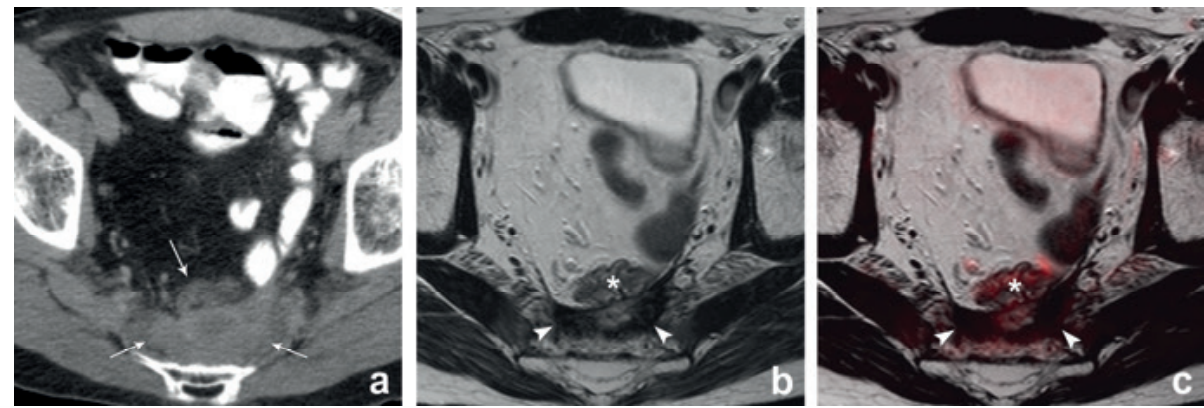

Figure 5. CT, T2-weighted and T2W + DWI fusion images of a male patient who had previously undergone an abdominoperineal resection for a distally located rectal tumour. On CT (a) there is a presacrally located soft tissue mass. On CT it is not possible to discriminate between post-operative scar tissue and recurrent tumour. On the corresponding MRI (b) there is an area of hypointense signal intensity indicating fibrosis (arrowheads). Located anterior to this fibrosis, there is a bowel loop $\left({ }^{*}\right)$ This bowel loop was not opacified by the oral contrast on CT, because it was located distally from the patient's ileostomy. On the fusion images (c) there are no areas of high signal intensity, suggestive of recurrent tumour. Follow-up imaging revealed no tumour recurrence. 


\begin{tabular}{|c|c|c|c|c|}
\hline \multirow{2}{*}{ Patient $\mathbf{N}^{\circ}$} & \multirow{2}{*}{ CT findings } & \multicolumn{2}{|c|}{ MRI (+ DWI) } & \multirow{2}{*}{$\begin{array}{c}\text { Outcome } \\
\text { (Ref. standard) }\end{array}$} \\
\hline & & R1 & $\mathbf{R 2}$ & \\
\hline 2 & - & - & - & - \\
\hline 5 & Inconclusive & + & + & + \\
\hline 12 & Inconclusive & - & - & - \\
\hline 14 & - & - & - & - \\
\hline 18 & Inconclusive & + & + & + \\
\hline 26 & Inconclusive & - & - & - \\
\hline 30 & Inconclusive & - & - & - \\
\hline 33 & + & + & + & + \\
\hline 35 & Inconclusive & + & + & + \\
\hline 36 & Inconclusive & + & + & + \\
\hline 40 & Inconclusive & - & - & - \\
\hline \multicolumn{5}{|c|}{$\begin{array}{l}\text { R1: reader } 1 \text {, a senior } \mathrm{GI} \text { radiologist with } 13 \text { years specific pelvic MR expertise. R2: reader } 2 \text {, a junior } \mathrm{GI} \\
\text { radiologist with } 3 \text { years pelvic MR expertise. } \\
\text { + indicates a local tumour recurrence } \\
\text { - indicates no recurrence }\end{array}$} \\
\hline
\end{tabular}

\section{Discussion}

The aim of this study was to assess the performance of MRI for the diagnosis of locally recurrent rectal cancer in patients with a clinical suspicion of a recurrence after rectal cancer surgery and to evaluate whether the addition of diffusionweighted imaging and/or image fusion of standard MRI and DWI improves its performance.

Our results indicate that standard MRI on its own has a high accuracy for the diagnosis of recurrent tumour in this specific group of patients. The addition of DWI does not significantly improve its performance. The high accuracy using only standard MRI could partly be due to the relatively high levels of expertise of the readers in this study. Furthermore, some patients had relatively large tumours, which were already correctly identified on morphological MR imaging. The benefit of DWI might be more pronounced for the detection of smaller tumours which are more difficult to detect within the fibrotic scar tissue. Results from a recent study by Syk et al. showed that mainly anastomotic recurrences - which tend to be smaller in 
size - are missed with imaging, again suggesting that the benefit from additional functional imaging may be higher in the detection of these small tumours. ${ }^{26}$ In our study we indeed observed that DWI was helpful in such a case: the less experienced radiologist detected one of the small recurrences on MRI + DWI which he had overlooked before (Figure 4). Larger patient studies are, however, required to further investigate and confirm the potential role of DWI in small tumours.

Of interest was the fact that adding DWI improved the interobserver agreement and reduced the number of false positives. DWI thus seems to increase the confidence of radiologists to rule out the presence of a recurrence (Figure 3 ). This a relevant finding, as it is known from previous studies that standard MRI generally tends to overestimate the presence of tumour within areas of fibrotic, postoperative scar tissue. ${ }^{14-16,27}$ De Lange et al. analysed the signal intensities of areas suspected for local tumour recurrence on T2-weighted MRI and reported equally low signal intensities for areas of desmoplastic reaction containing tumour and areas of desmoplasia only. ${ }^{27}$ It is especially in these cases that the combination of morphological information from MRI and the functional information from DWI can be beneficial in the diagnosis of recurrent tumour growth. Fibrotic tissue typically has a low cellular density and a large interstitial space, which results in low signal intensity on high b-value (b1000) diffusion images. Conversely, tumoural areas have a relatively high cellular density, which will result in high signal on DWI. ${ }^{28}$ Our findings are in concordance with a previous report from Nishie et al, who compared standard MRI to DWI and fusion images for the diagnosis of pelvic tumour recurrences in a group of 28 patients with a variety of primary pelvic malignancies. ${ }^{20}$ They found the fusion images of T2-weighted MRI and DWI to perform the best with an AUC of 0.95. The main gain from the addition of DWI was the improved specificity from $47 \%$ on standard to MRI to $72 \%$ with $\mathrm{DWI}$, indicating that false positivity could be reduced by more than $20 \%$.

As compared to the separate evaluation of the standard MRI and diffusion images, the addition of image fusion did not improve the performance of the readers in our study. Both readers, however, experienced the fusion images to be helpful for a better understanding of the anatomical correlation between standard MRI and DWI. MRI is presently mainly used as a pre-operative roadmap for surgeons to determine the resectability of recurrence in patients who are potential candidates for curative salvage surgery. ${ }^{14}$ In this regard, image fusion of standard MRI and DWI might be beneficial and should be addressed by further studies.

In our study, eleven patients had undergone CT imaging prior to MRI. In eight (73\%) cases CT showed equivocal findings and patients had to be referred for further imaging. The readers were not aware of the fact that a CT had been performed previous to the MRI, nor were any of the CT findings revealed. In this 
study setting, all eight patients were correctly diagnosed by both readers, both on standard MRI and on MRI plus DWI, suggesting that MRI has superior sensitivity and specificity compared to CT. The limitation of CT remains its inferior soft tissue contrast as compared to MRI. Previous studies that have compared CT and MRI for evaluation of local recurrences have reported similar superiority of MRI, both for the detection of recurrent tumour in this specific group of patients as well as for the assessment of tumour resectability. ${ }^{29,30}$ In the current study, MRI without contrast was used. Some authors have suggested the use of dynamic contrast enhanced (DCE) Gadolinium sequences for a better diagnosis of recurrent tumour. ${ }^{31,32}$ Results are, however, somewhat conflicting as others have shown little improvement for DCE compared to standard morphological MRI. ${ }^{33}$

Our study is limited because of its retrospective nature. Furthermore, we only observed a trend towards an improved performance for MRI + DWI, which could partly be due to the small sample size and the relatively high level of experience of both readers in our study. The results of our study are, however, promising and therefore warrant further prospective studies to confirm the value of $\mathrm{MRI}+\mathrm{DWI}$ in larger patient groups and in a general clinical setting.

In conclusion, this study shows that standard MRI on its own has a high accuracy for the diagnosis of locally recurrent rectal cancer. Addition of DWI does not significantly improve its performance. However, addition of DWI might help radiologists to more confidently rule out the presence of a recurrence. Moreover, the use of DWI can increase interobserver agreement. MRI is therefore promising as a tool to detect local recurrences after rectal cancer surgery in the group of patients with a high clinical suspicion for recurrence. Fusion images of MRI + DWI do not further improve the diagnostic accuracy. 


\section{References}

1. Jeffery $M$, Hickey $B E$, Hider PN. Follow-up strategies for patients treated for non-metastatic colorectal cancer. Cochrane Database Syst Rev 2007; 24(1):CD002200.

2. Kusters M, Marijnen CA, van de Velde $\mathrm{CJ}$, et al. Patterns of local recurrence in rectal cancer; a study of the Dutch TME trial. Eur J Surg Oncol 2010;36(5):470-476.

3. Caricato M, Borzomati D, Ausania F, Valeri S, Rosignoli A, Coppola R. Prognostic factors after surgery for locally recurrent rectal cancer: an overview. Eur J Surg Oncol 2006; 32(2):126-132.

4. Desch CE, Benson AB 3rd, Somerfield MR, et al.; American Society of Clinical Oncology. Colorectal cancer surveillance: 2005 update of an American Society of Clinical Oncology practice guideline. J Clin Oncol 2005;23(33):8512-8519.

5. Glimelius B, Påhlman L, Cervantes A; ESMO Guidelines Working Group. Rectal cancer: ESMO Clinical Practice Guidelines for diagnosis, treatment and follow-up. Ann Oncol 2010; 21(Suppl 5):v82-86.

6. Titu LV, Nicholson AA, Hartley JE, Breen DJ, Monson JR. Routine follow-up by magnetic resonance imaging does not improve detection of resectable local recurrences from colorectal cancer. Ann Surg 2006;243(3):348-352.

7. Schaefer $\mathrm{O}$, Langer M. Detection of recurrent rectal cancer with CT, MRI and PET/CT. Eur Radiol 2007;17(8):2044-54.

8. Valentini V, Aristei C, Glimelius B, et al. Multidisciplinary Rectal Cancer Management: 2nd European Rectal Cancer Consensus Conference (EURECA-CC2). Radiother Oncol 2009;92(2):148-163.

9. Takeuchi O, Saito N, Koda K, Sarashina H, Nakajima N. Clinical assessment of positron emission tomography for the diagnosis of local recurrence in colorectal cancer. Br J Surg 1999; 86(7):932-937.

10. Flamen $P$, Stroobants $S$, Van Cutsem E, et al. Additional value of whole-body positron emission tomography with fluorine-18-2-fluoro-2-deoxy-D-glucose in recurrent colorectal cancer. J Clin Oncol 1999;17(3):894-901.

11. Even-Sapir E, Parag $\mathrm{Y}$, Lerman $\mathrm{H}$, et al. Detection of recurrence in patients with rectal cancer: PET/CT after abdominoperineal or anterior resection. Radiology 2004;232(3):815-822.

12. Vliegen RF, Beets-Tan RG, Vanhauten B, et al. Can an FDG-PET/CT predict tumor clearance of the mesorectal fascia after preoperative chemoradiation of locally advanced rectal cancer? Strahlenther Onkol 2008;184(9):457-464.

13. Dresen RC, Kusters M, Daniels-Gooszen AW, et al. Absence of tumor invasion into pelvic structures in locally recurrent rectal cancer: prediction with preoperative $M R$ imaging. Radiology 2010;256(1):143-150.

14. Dresen RC, Beets GL, Rutten $\mathrm{HJ}$, et al. Locally advanced rectal cancer: MR imaging for restaging after neoadjuvant radiation therapy with concomitant chemotherapy. Part I. Are we able to predict tumor confined to the rectal wall? Radiology 2009;252(1):71-80.

15. Kuo $\mathrm{LJ}$, Chern MC, Tsou MH, et al. Interpretation of magnetic resonance imaging for locally advanced rectal carcinoma after preoperative chemoradiation therapy. Dis Colon Rectum 2005;48(1):23-28.

16. Barbaro B, Vitale R, Leccisotti L, et al. Restaging locally advanced rectal cancer with MR imaging after chemoradiation therapy. Radiographics 2010;30(3):699-716.

17. Koh DM, Collins DJ. Diffusion-weighted MRI in the body: applications and challenges in oncology. AJR Am J Roentgenol 2007;188(6):1622-1635.

18. Namimoto T, Awai K, Nakaura T, Yanaga Y, Hirai T, Yamashita Y. Role of diffusion-weighted imaging in the diagnosis of gynecological diseases. Eur Radiol 2009;19(3):745-760.

19. Rao SX, Zeng MS, Chen CZ, Li RC, Zhang SJ, Xu JM, Hou YY. The value of diffusion-weighted imaging in combination with T2-weighted imaging for rectal cancer detection. Eur J Radiol 2008;65(2):299-303.

20. Nishie A, Stolpen AH, Obuchi M, Kuehn DM, Dagit A, Andresen K. Evaluation of locally recurrent pelvic malignancy: performance of T2- and diffusion-weighted MRI with image fusion. J Magn Reson Imaging 2008;28(3):705-713. 
21. Tsushima Y, Takano A, Taketomi-Takahashi A, Endo K. Body diffusion-weighted MR imaging using high b-value for malignant tumor screening: usefulness and necessity of referring to T2-weighted images and creating fusion images. Acad Radiol 2007;14(6):643-650.

22. Takahara T, Imai Y, Yamashita T, Yasuda S, Nasu S, Van Cauteren M. Diffusion weighted whole body imaging with background body signal suppression (DWIBS): technical improvement using free breathing, STIR and high resolution 3D display. Radiat Med 2004;22(4):275-282.

23. Rosset A, Spadola L, Ratib O. OsiriX: an open-source software for navigating in multidimensional DICOM images. J Digit Imaging 2004;17(3):205-216.

24. DeLong ER, DeLong DM, Clarke-Pearson DL. Comparing the areas under two or more correlated receiver operating characteristic curves: a nonparametric approach. Biometrics 1988; 44(3):837-845.

25. Cohen J. Weighted kappa: nominal scale agreement with provision for scaled disagreement or partial credit. Psychol Bull 1986;70:213-220.

26. Syk E, Torkzad MR, Blomqvist L, Nilsson PJ, Glimelius B. Local recurrence in rectal cancer: anatomic localization and effect on radiation target. Int J Radiat Oncol Biol Phys 2008;72(3):658-664

27. de Lange EE, Fechner RE, Wanebo HJ. Suspected recurrent rectosigmoid carcinoma after abdominoperineal resection: MR imaging and histopathologic findings. Radiology 1989;170(2):323328.

28. Vandecaveye V, De Keyzer F, Nuyts S, et al. Detection of head and neck squamous cell carcinoma with diffusion weighted MRI after (chemo)radiotherapy: correlation between radiologic and histopathologic findings. Int J Radiat Oncol Biol Phys 2007;67(4):960-971.

29. Blomqvist L, Holm T, Goranson H, Jacobsson H, Ohlsen H, Larsson SA. MR imaging, CT and CEA scintigraphy in the diagnosis of local recurrence of rectal carcinoma. Acta Radiol 1996;37(5):779-784.

30. Beets-Tan RG, Beets GL, Borstlap AC, Oei TK, Teune TM, von Meyenfeldt MF, van Engelshoven JM. Preoperative assessment of local tumor extent in advanced rectal cancer: CT or high-resolution MRI? Abdom Imaging 2000;25(5):533-541.

31. Kinkel K, Tardivon AA, Soyer P, Spatz A, Lasser P, Rougier P, Vanel D. Dynamic contrast-enhanced subtraction versus T2-weighted spin-echo MR imaging in the follow-up of colorectal neoplasm: a prospective study of 41 patients. Radiology 1996;200(2):453-458

32. Torricelli P, Pecchi A, Luppi G, Romagnoli R. Gadolinium-enhanced MRI with dynamic evaluation in diagnosing the local recurrence of rectal cancer. Abdom Imaging 2003;28(1):19-27

33. Blomqvist L, Fransson P, Hindmarsh T (1998). The pelvis after surgery and radio-chemotherapy for rectal cancer studied with Gd-DTPA-enhanced fast dynamic MR imaging. Eur Radiol 1998;8(5):781787. 


\title{
DISCUSSION AND RECOMMENDATIONS
}

\section{SUMMARY / SAMENVATTING}

\author{
DANKBETUIGINGEN \\ CURRICULUM VITAE \\ LIST OF PUBLICATIONS
}

CHAPTER 


\section{Discussion and Recommendations}

\section{Discussion}

Response evaluation of locally advanced rectal cancers after chemoradiation treatment is only useful if it would impact clinical management. Until recently, this has not been a relevant issue, since all patients would receive surgery regardless of their response to preoperative treatment. However, the current trend in therapy is to go for minimally invasive, organ saving treatment approaches to reduce treatment morbidity. In rectal cancer patients that have responded well to preoperative treatment, transanal endoscopic microsurgery or even deferral from surgery could be promising alternatives to standard surgical resection. Although at the moment of writing these treatments are still controversial, multicenter observational trials are running to investigate their efficacy. This opens the debate whether imaging tools are available that can accurately assess the treatment response. In order to be able to stratify the good and complete responding patients into organ preserving treatment options, reliable selection of these patients is critical. So far, response after chemoradiotherapy has mainly been assessed by clinical examination (digital rectal examination and endoscopy). The role of imaging was traditionally limited because of difficulties in evaluating lymph nodes and identifying small residual disease. The aim of this thesis was to investigate whether functional modern MR techniques such as diffusion-weighted MRI (DWI) and lymph node specific contrast-enhanced MRI will improve the clinical selection of patients who may be eligible to undergo organ saving treatments as an alternative to standard surgery.

\section{Part I-Tumour response}

We have shown in chapter 2 that the addition of diffusion-weighted MRI to conventional T2-weighted MRI can improve the identification of rectal tumours that have completely responded to preoperative chemoradiotherapy. Apparently, the functional information of DWI better indicates the presence or absence of vital tumour remnants within the irradiated fibrotic tumour bed than the morphological information of conventional MRI. DWI analyses differences in the motion ('diffusion') of water protons, mainly in the extracellular tissue space. In tissues with a low cellular density there is ample extracellular space and water protons can move relatively freely. These moving water protons cause a decrease in signal intensity on diffusion-weighted MR images. Fibrosis consists of a loose fibre network of connective tissue. The limiting effect of these fibres reduces the water diffusion to some extent. This restrictive effect is, however, not as pronounced as in 
the remaining tumour areas, in which the proliferation of cancer cells still forms abundant barriers to water movement. As a result, malignant tumours remain high in signal intensity, which makes them stand out within the suppressed MR signal of the surrounding tissues and fibrosis.

Despite the significant improvement of MRI response assessment when using DWI, the sensitivity for discrimination of complete responders in our study in chapter 2 was only $52-64 \%$, indicating that in a substantial number of cases the presence of residual tumour was still overestimated. We believe that this effect can be partly attributed to the way the reading design was constructed: simultaneous exposure of DWI and T2-weighted sequences to the reader. This way of image evaluation was chosen as it represents the way diffusion images will generally be read in standard clinical practice, i.e. side by side with T2-weighted images combining functional with morphological data. Yet, by doing so it seems that radiologists are very much influenced by their interpretation of the morphological findings on T2-weighted images, which is a more familiar process for them. For the present generation radiologists, integrating functional with morphological information in their diagnostic decision making process is still a new concept. As always when new procedures or new imaging tools are introduced, one needs time to accept that its implementation would be beneficial. With regards to our reading design, in the cases where a massive fibrosis is visualised on T2-weighted MRI, radiologists are used to overstage residual disease knowing that in oncology it is best to err on the 'safe' side. Even when the diffusion images would suggest otherwise, they tend to be reluctant to follow this information. We could confirm that these interpretation errors reduced when DWI images were read independent and blinded to the T2-weighted MRI. Chapter 3 shows that if the volume of the areas of high signal intensity on DWI (indicating tumour) was objectively measured, without relating the DWI findings to the T2W images, the sensitivity for identification of complete responders increased to over $90 \%$. We recognise that volumetry is time consuming and hard to implement in a busy clinical practice. We, however, believe that these volumetric measurements are not necessarily required. In the end, manually performed volume measurements are still based on the visual perception of the presence or absence of high signal areas on DWI. As such, a visual interpretation of whether or not a high signal area (indicating residual tumour) is present on DWI may well be sufficient and it may not be obligatory to measure its volume. Computer Aided Diagnosis (CAD) systems, which allow for semi-automated analyses, might also be of supplementary value, although at present these systems are in an early stage of development and do not yet account for the interpretation pitfalls of DWI, such as T2 shine-through. Hence, radiologists' interpretation skills 
will still be required and radiologists will need to be trained to understand the pitfalls of DWI image interpretation.

Quantitative assessment of the tumour apparent diffusion coefficient was not found useful in the identification of complete responders. So far, only a few research groups have reported promising results for $A D C$ in the assessment of a complete tumour response and there is no strong evidence that $A D C$ is truly helpful in response evaluation. ${ }^{1-5}$ Furthermore, different authors have acknowledged that it remains very difficult to discriminate true complete responders from 'nearly complete responders' in whom only a few isolated tumour cells remain after CRT. ADC measurements are probably better suited for gross distinction between 'favourable' and 'unfavourable' tumour response groups, as was also demonstrated by previous work. ${ }^{6-8}$ Furthermore, ADC has shown promise to predict whether patients are likely to respond to treatment, early (after one or two weeks of chemoradiation treatment) during the treatment course. ${ }^{6,9}$ The use of ADC as a tumour biomarker could thus help to allow a first selection of those patients that are likely to show a good response to chemoradiation treatment. To establish the final diagnosis of a good or complete tumour response after completion of chemoradiation treatment, other or additional tools will, however, likely be required.

When considering the use of ADC for response evaluation, one should also bear in mind that $A D C$ values show large variations caused by differences in MR equipment and DWI sequence parameters. ${ }^{10}$ Furthermore, we found that the way we analyse the DWI data also influences $A D C$ values. The effect of different methods of ADC measurements was the focus of the study described in chapter 4 . This study proves that ADC values are significantly affected by the number and size of regions of interest used to obtain ADC measurements and that the best way to obtain tumour ADC values is by means of a complete volumetric approach. These issues should be taken into account and efforts should be made to standardise imaging and analysis methods.

\section{Part II - Lymph nodes}

If a local excision or wait-and-see strategy is being considered, it is critical to know whether all previously metastatic lymph nodes have been sterilised. Imaging has so far not been sufficiently accurate, because morphological imaging could only rely on nodal size as the main criterion to discriminate metastatic nodes. Size is a very poor predictor for rectal cancer nodes, since over $50 \%$ of the nodal metastases are known to occur in nodes smaller than $5 \mathrm{~mm}$, which is a cut-off size frequently applied in clinical practice. ${ }^{11}$ Interestingly, more favourable results have been 
reported for nodal restaging after chemoradiotherapy. ${ }^{12,13}$ After chemoradiation many of the mainly smaller sized irradiated nodes disappear and the mean nodal size decreases. Given the lower prevalence of node positive disease after CRT, the nodes that remain larger in size are far more likely to be metastatic. Hence, size criteria are better applicable in the restaging setting. Nevertheless, size-based nodal staging - also after chemoradiotherapy - remains insufficiently accurate to allow a safe clinical decision making process. Morphological MR criteria such as the nodal border contour and signal homogeneity have shown additional value. These criteria, however, are difficult to assess in the smaller sized $(2-3 \mathrm{~mm})$ nodes and therefore particularly less applicable in the restaging setting, wherein most of the nodes are small. ${ }^{14,15}$ The question is whether functional or metabolic imaging can improve the performance of morphological imaging. Metabolic 18F-fluorodeoxyglucose positron emission tomography (FDG-PET) imaging is not useful in this regard, since most of the mesorectal nodes are too small to detect with PET. Moreover, nodes in the proximity of the tumour will be obscured due to artefacts caused by the intense uptake of FDG in the tumour. ${ }^{16}$ Studies in pelvic nodes have shown disappointing performance for FDG-PET. ${ }^{17,18} 19$ In the study in chapter 5 we intended to investigate whether diffusion-weighted MRI can be of additional value for nodal staging. Although significant differences in ADC between benign and metastatic lymph nodes were observed, the addition of ADC to standard T2-weighted MRI did not contribute to an improved diagnostic performance. This was partly because the variations in $A D C$ measurements between the two observers exceeded the difference in $A D C$ between the benign and malignant nodes. Moreover, nodal characterization by $A D C$ is very challenging in the smaller sized nodes, in which it will be even more difficult to perform representative and reproducible measurements. Given the fact that in rectal cancer the majority of the nodes are smaller than $5 \mathrm{~mm}, A D C$ quantification does not seem the way to go. Even if proven otherwise, $A D C$ quantification in patients with rectal cancer - who can present with well up to 20 nodes, regardless their nodal stage - would be very time consuming and not practical for implementation in busy practice. Hence, we will have to expand our view and seek the solution in 'molecular' imaging techniques with contrast agents that more specifically target the lymph nodes.

The only method that so far has proven reliable is MRI enhanced with the lymph node specific contrast agent Ultrasmall Superparamagnetic Parts of Iron Oxide (USPIO). ${ }^{20,21,22}$ The uptake of USPIO in the nodes reflects the way tumour spreads in the lymphatic system. It accumulates in macrophages within healthy lymphoid tissue, while in tumour bearing nodes there is no accumulation owing to the absence of these macrophages. USPIO, however, is not available for clinical use, because the contrast has been withdrawn from its approval by the food and drug 
administration. In 2002, Herborn et al. investigated the use of a new gadoliniumbased contrast agent, gadofosveset trisodium. They injected gadofosveset interstitially in an animal tumour model and found that it resulted in a more or less selective enhancement of normal, non-tumoural lymphoid tissue. ${ }^{23}$ Subsequently, Lahaye et al. performed a small human pilot study in 10 rectal cancer patients demonstrating that intravenous administration of gadofosveset also results in uptake in healthy lymph nodes. ${ }^{24}$ The exact uptake mechanism of gadofosveset by the lymphoid system is not yet fully understood. It is, however, known that gadofosveset binds reversibly to human albumin. We hypothesise that when the albumin-gadofosveset compound within the vasculature of the nodes is fully saturated, the remaining unbound fraction of gadofosveset diffuses into the extravascular space where it binds to albumin. In its albumin-bound macromolecular form gadofosveset particles cannot reflux back to the blood lumen and accumulate within the nodal interstitium, which causes a T1-shortening and subsequent high signal intensity of the healthy lymph nodes on T1-weighted MRI. Tumoural nodes have leaky microvessels through which the albumin-gadofosveset compound will reflux back and forth from the interstitial into the blood compartment. As a result there is no accumulation of gadofosveset in the interstitial space and metastatic nodes remain dark on MRI.

In the study presented in chapter 6 we have confirmed that intravenous injection of gadofosveset results in a selective signal enhancement in nonmetastatic lymph nodes. The most important gain from the gadofosveset enhanced MRI was that the number of false negative assessments on post-CRT MRI could considerably be reduced compared to standard MRI. In the per patient analyses, the mean sensitivity for the two readers after CRT improved from $68 \%$ on standard MRI to $84 \%$ on the gadofosveset images and the NPV improved from $71 \%$ to $87 \%$. Although we acknowledge that our study was not powered to allow for definitive conclusions on a per patient basis, these preliminary results indicate that when using gadofosveset the ypNO patients can be more accurately selected. This could have a significant clinical impact as it implicates that patients may more safely be selected for organ saving treatments with only a minimal risk for understaging (and thus undertreatment). In addition, the use of gadofosveset greatly reduces the number of false positives compared to standard MR imaging. In the group of patients that underwent chemoradiotherapy, standard MRI overstaged $30-43 \%$ of the ypNO patients as being ypN+. With the addition of gadofosveset, the overstaging errors could be reduced to approximately $15 \%$. Our study outcome is encouraging in such a way that it seems worthwhile to further investigate whether lymph node specific MR contrasts can help us in solving the dilemma of nodal imaging. The criteria for gadofosveset-enhanced MRI established in our pilot study will need to 
be tested in a second and larger patient cohort to evaluate its value on a per patient level and by readers with different levels of expertise. Finally, the use of gadofosveset should be incorporated into therapeutic clinical trials to establish its true clinical value and potential impact on patient outcome.

\section{Part III - Follow-up}

During the surveillance of patients after surgical treatment, the role of MRI has so far been restricted to assess tumour resectability in patients with a proven local tumour recurrence. The results of the study in chapter 8 show that MRI may also be used for the primary detection of a local tumour re-growth in the selected group of patients who have a high suspicion of recurrence based on a rising CEA levels and/or clinical symptoms. In this particular subgroup of patients, the use of MRI as a single examination may be more time- and cost-effective than the conventional diagnostic approach in which patients first undergo CT and - if equivocal - undergo additional FDG-PET and/or MR imaging. Moreover, the addition of DWI to conventional MRI can help identify tumour re-growth within areas of post-treatment fibrosis, which is difficult to assess with standard imaging methods. Morphological changes in the fibrotic tissue that could indicate a local tumour re-growth can be confirmed by signal intensity changes observed on DWI, which information - when combined may improve our performance for early detection of small recurrences and thus increase the chance that the recurrent tumour may still be curatively treated. This was also the rationale behind the surveillance scheme of our wait-and-see patients included in the regional clinical trial. This trial, recently published in the journal of clinical oncology ${ }^{25}$ has so far included 21 patients with only one local recurrences at 2-3 yrs follow up, treated with salvage surgery and still alive without disease. We monitor these patients very closely with clinical examinations, laboratory testing and CT for distant metastases screening, together with an endoscopy and rectal MRI for local recurrence detection, performed every 3 months in the first year and every 6 months during the following years. The study in chapter 7 gave us insight into how the MR morphology of the irradiated tumour bed evolves over time in case of a sustained complete response and in case of a local recurrence. The results show that in patients with a sustained complete response, the morphology of the normalised or fibrotic rectal wall remains unchanged over the years. Conversely, when the signal intensity, shape or volume of the initial tumour area starts to change, this should alert us of a potential tumour re-growth. In our experience, in particular signal intensity changes on consecutive DWI scans were powerful signs to signal the development of a local tumour re-growth as these could be observed even before morphological changes occurred. To confidently continue a wait-and- 
see policy, it is important to be able to detect any potential recurrences as early as possible in order to offer these patients salvage surgery. The true clinical value of DWI in this specific setting will need to be established by further studies.

\section{Future perspectives}

Ongoing developments in the treatment of rectal cancer lead to new diagnostic challenges. It may in the future not only be important to evaluate the final treatment response after completion of chemoradiation treatment, but also to predict response early during the course of chemoradiotherapy. Some patients do not respond well to CRT and show only a limited tumour downsizing. These patients benefit little from the addition of neoadjuvant treatment, but are still exposed to the associated morbidity. If we would have a way to establish how a tumour will behave early after onset of treatment, this might enable us to identify who are the patients that are likely to benefit from the CRT and who will not, in order to intensify the preoperative treatment in the first group and alter the treatment in the latter. This could result in more personalised medicine driven by imaging findings. FDG-PET is one potential tool to monitor response. It has shown particular value in predicting tumour response early (1-2 weeks) after onset of treatment. ${ }^{4,26}$ Diffusion-weighted MRI is another tool that could be promising for early response assessment as it can monitor early changes in the cellular tumour behaviour after initiation of treatment. FDG-PET is routine now in image guided, intensity modulated radiotherapy planning (IMRT). DWI, however, still needs to prove its value. However, given the fact that DWI reflects tumour cellularity while PET reflects tumour metabolism, DWI has potential to complement PET in local and distant visualization of the tumour burden. ${ }^{27,28}$ Especially in patients with metastatic liver disease who are candidates for curative (multimodality) liver treatment, we can foresee an important role for DWI to support PET imaging in identifying the full extent of liver disease. This combined use of metabolic, molecular and functional imaging techniques needs to be further explored and significant improvement is still to be expected. The time that imaging provides information only on tumour morphology has passed and detection of small volume disease will be made possible through multimodality, multiparametric imaging technology. 


\section{Recommendations}

Modern MR imaging is a powerful tool for evaluating the response to CRT in patients with locally advanced rectal cancer. Its main strength is its ability to combine morphological with functional data, such as that of diffusion-weighted MRI or lymph node specific contrast enhanced MRI. The basic sequence of a restaging MRI after CRT remains a standard T2-weighted MRI. T2W images in multiple planes allow a first discrimination between the 'poor responding' and the 'well responding' patients. The poor responding patients in whom a clear residual tumour mass (yT34 ) is still present may immediately be referred for surgical treatment without the need for additional imaging evaluation. This leaves the 'well responding' group for further imaging. If further discrimination between the good responders (ypT1-2) and complete responders (ypT0) is desired because it would impact the treatment choice, standard T2W MRI is no longer sufficient. Here, diffusion-weighted MRI can have a role. The combination of MRI + DWI can provide a more valid diagnosis of the presence of residual tumour within the (fibrotic) tumour bed after chemoradiation treatment and help select the patients with a small tumour remnant that can be considered for a local excision. Furthermore, the risk for false negative results (i.e. understaging of patients with residual tumour) with DWI is lower than with T2W images only. In other words, if DWI tells us that a patient has not completely responded, we should not expose the patient to undertreatment by deferring him from surgery. The 'grey zone' remains the $15-50 \%$ of patients with a complete response in whom - even with DWI - the presence of tumour is overestimated. It is in these cases that specific attention should be given to the integrated information from clinical examinations, endoscopy, biopsy, MRI and DWI images. A normalised rectal wall and negative tumour biopsies at endoscopy together with the absence of residual high signal intensity on DWI can increase the confidence in establishing a final diagnosis of a complete response. For the assessment of lymph nodes, there is not yet a good solution. DWI is not useful for the characterization of lymph nodes. Lymph node specific MR contrast agents seem the way to go. The current evidence for gadofosveset contrast is only from one single center study and the availability of the contrast agent is still limited. Nevertheless, the use of gadofosveset contrast enhanced MRI for nodal staging in rectal cancer is so promising that it justifies further exploration in this area. 


\section{References}

1. Song I, Kim SH, Lee SJ, et al: Value of diffusion-weighted imaging in the detection of viable tumour after neoadjuvant chemoradiation therapy in patients with locally advanced rectal cancer: comparison with T2-weighted and PET/CT imaging. Br J Radiol 2012;85:577-86

2. Kim SH, Lee JM, Hong SH, et al: Locally advanced rectal cancer: added value of diffusion-weighted MR imaging in the evaluation of tumor response to neoadjuvant chemo- and radiation therapy. Radiology 2009;253:116-25

3. Kim SH, Lee JY, Lee JM, et al: Apparent diffusion coefficient for evaluating tumour response to neoadjuvant chemoradiation therapy for locally advanced rectal cancer. Eur Radiol 2011;21:987-95

4. Lambrecht M, Deroose C, Roels S, et al: The use of FDG-PET/CT and diffusion-weighted magnetic resonance imaging for response prediction before, during and after preoperative chemoradiotherapy for rectal cancer. Acta Oncol 2010;49:956-963

5. Lambregts DMJ, Vandecaveye V, Barbaro B, et al: Diffusion-weighted MRI for selection of complete responders after chemoradiation for locally advanced rectal cancer: a multicenter study. Ann Surg Oncol 2011;18:2224-31

6. Sun YS, Zhang XP, Tang L, et al: Locally advanced rectal carcinoma treated with preoperative chemotherapy and radiation therapy: preliminary analysis of diffusion-weighted MR imaging for early detection of tumor histopathologic downstaging. Radiology 2010;254:170-8

7. Dzik-Jurasz A, Domenig C, George M, et al: Diffusion MRI for prediction of response of rectal cancer to chemoradiation. Lancet 2002;360:307-8

8. Jung $\mathrm{SH}$, Heo SH, Kim JW, et al: Predicting response to neoadjuvant chemoradiation therapy in locally advanced rectal cancer: diffusion-weighted 3 Tesla MR imaging. J Magn Reson Imaging 2012;35:110-6

9. Lambrecht M, Vandecaveye V, De Keyzer F, et al: Value of Diffusion-Weighted Magnetic Resonance Imaging for Prediction and Early Assessment of Response to Neoadjuvant Radiochemotherapy in Rectal Cancer: Preliminary Results. Int J Radiat Oncol Biol Phys 2012;82:863-70

10. Padhani AR, Liu G, Koh DM, et al: Diffusion-weighted magnetic resonance imaging as a cancer biomarker: consensus and recommendations. Neoplasia 2009;11:102-25

11. Wang C, Zhou Z, Wang Z, et al: Patterns of neoplastic foci and lymph node micrometastasis within the mesorectum. Langenbecks Arch Surg 2005;390:312-8

12. Lahaye MJ, Beets GL, Engelen SM, et al: Locally advanced rectal cancer: MR imaging for restaging after neoadjuvant radiation therapy with concomitant chemotherapy. Part II. What are the criteria to predict involved lymph nodes? Radiology 2009;252:81-91

13. Suppiah A, Hunter IA, Cowley J, et al: Magnetic resonance imaging accuracy in assessing tumour down-staging following chemoradiation in rectal cancer. Colorectal Dis 2009;11:249-53

14. Brown G, Richards CJ, Bourne MW, et al: Morphologic Predictors of Lymph Node Status in Rectal Cancer with Use of High-Spatial-Resolution MR Imaging with Histopathologic Comparison. Radiology 2003;227:371-377

15. Kim JH, Beets GL, Kim JH, et al: High-resolution MR imaging for nodal staging in rectal cancer: are there any criteria in addition to the size? Eur J Radiol 2004;52:78-83

16. Koh DM, Brown G, Husband JE: Nodal staging in rectal cancer. Abdom Imaging 2006;31:652-9

17. Ono K, Ochiai R, Yoshida T, et al: Comparison of diffusion-weighted MRI and 2-[fluorine-18]-fluoro-2deoxy-D-glucose positron emission tomography (FDG-PET) for detecting primary colorectal cancer and regional lymph node metastases. J Magn Reson Imaging 2009;29:336-40

18. Kim DJ, Kim JH, Ryu YH, et al: Nodal staging of rectal cancer: high-resolution pelvic MRI versus (1)FFDGPET/CT. J Comput Assist Tomogr 2011;35:531-4

19. Abdel-Nabi H, Doerr RJ, Lamonica DM, et al: Staging of primary colorectal carcinomas with fluorine18 fluorodeoxyglucose whole-body PET: correlation with histopathologic and CT findings. Radiology 1998;206:755-60 
20. Will O, Purkayastha S, Chan C, et al: Diagnostic precision of nanoparticle-enhanced MRI for lymphnode metastases: a meta-analysis. Lancet Oncol 2006;7:52-60

21. Koh DM, Brown G, Collins DJ: Nanoparticles in rectal cancer imaging. Cancer Biomark 2009;5:89-98

22. Lahaye MJ, Engelen SM, Kessels AG, et al: USPIO-enhanced MR imaging for nodal staging in patients with primary rectal cancer: predictive criteria. Radiology 2008;246:804-11

23. Herborn CU, Lauenstein TC, Vogt FM, et al: Interstitial MR lymphography with MS-325: characterization of normal and tumor-invaded lymph nodes in a rabbit model. AJR Am J Roentgenol 2002;179:1567-72

24. Lahaye MJ, Beets GL, Engelen SME, et al: Gadofosveset Trisodium (Vasovist ${ }^{\circledR}$ ) enhanced MR lymph node detection: initial observations. The Open Magnetic Resonance Journal 2009;2:1-5

25. Maas M, Beets-Tan RG, Lambregts DM, et al: Wait-and-See Policy for Clinical Complete Responders After Chemoradiation for Rectal Cancer. J Clin Oncol 2011;29:4633-40

26. Janssen MH, Ollers MC, Riedl RG, et al: Accurate Prediction of Pathological Rectal Tumor Response after Two Weeks of Preoperative Radiochemotherapy Using (18)F-Fluorodeoxyglucose-Positron Emission Tomography-Computed Tomography Imaging. Int J Radiat Oncol Biol Phys 2010;77:392-99

27. Heusner TA, Kuemmel S, Koeninger A, et al: Diagnostic value of diffusion-weighted magnetic resonance imaging (DWI) compared to FDG PET/CT for whole-body breast cancer staging. Eur J Nucl Med Mol Imaging 2010;37:1077-86

28. Stecco A, Romano G, Negru M, et al: Whole-body diffusion-weighted magnetic resonance imaging in the staging of oncological patients: comparison with positron emission tomography computed tomography (PET-CT) in a pilot study. Radiol Med 2009;114:1-17 


\section{Summary}

The aim of this thesis was to evaluate whether and how diffusion-weighted MRI and gadofosveset-enhanced MRI can benefit the clinical selection of patients for tailoring of treatment into organ saving treatments. The thesis was subdivided into three parts: tumour response evaluation, nodal staging and follow-up.

\section{Part I - Tumour response}

Chapter 2 describes a multicenter study in which the diagnostic performance of diffusion-weighted $\mathrm{MRI}$ in addition to standard T2-weighted MRI for the identification of patients with a complete tumour response after chemoradiotherapy was analysed and compared with the performance of standard MRI only. For less experienced readers, the diagnostic performance improves significantly after addition of DWI. In particular the sensitivity for detection of complete responders improved by $16-52 \%$, indicating that underestimation of response can considerably be reduced compared to standard imaging.

The study in chapter 3 investigated the use of volumetric and ADC measurements on DWI for assessing tumour response and compared them to tumour volumetry performed on T2-weighted MRI. On postchemoradiation MRI, tumour volumetry based on areas showing high signal on high b-value diffusion images can accurately identify patients with a complete tumour response. It is significantly more accurate than conventional (T2W) tumour volumetry. Tumour volume reduction ratios calculated from pre- and posttreatment volumes derived from either standard MRI or DWI are equally accurate, although these latter approaches are far more time consuming than analysing the volume on post-treatment DWI only. ADC measurements and pretreatment volume measurements are not accurate to assess response.

In chapter 4 the effect of applying different types and sizes of 'Regions of Interest' for measuring tumour ADC was studied. The choice of ROIs significantly influences tumour ADC values and also affects the variability between different observers. ADC measurements obtained from the whole tumour volume are better reproducible than those obtained from a single tumour slice or a small tumour sample. Pretreatment measurements are better reproducible than those obtained after chemoradiation treatment. 


\section{Part II - Lymph nodes}

The study in chapter 5 analysed the use of DWI for the assessment of rectal cancer lymph nodes after chemoradiation treatment. Visual evaluation of DWI helps to improve the total number of nodes detected compared to standard T2-weighted MRI but is not useful for nodal characterization since both benign and malignant lymph nodes show equally high signal intensities. ADC values are significantly different between benign and metastatic lymph nodes, but the addition of ADC to standard T2-weighted imaging does not result in a significant improvement in diagnostic accuracy for nodal staging.

Chapter 6 describes the use of a new MR contrast agent, gadofosveset-trisodium, for the assessment of rectal cancer lymph nodes. Gadofosveset-enhanced MRI significantly improves the diagnostic performance compared to standard T2weighted MRI. Because only benign lymph nodes show uptake of gadofosveset contrast, these nodes show a marked increase in signal, whereas metastatic nodes remain dark. The nodal signal intensity combined with the 'nodal relief sign' result in high sensitivity and specificity for the differentiation between benign and metastatic nodes. The improvement compared to standard MRI is most pronounced for the primary staging of lymph nodes. For the restaging of nodes after chemoradiation therapy, the use of standard MRI only is already relatively accurate.

\section{Part III - Follow-up}

In chapter 7 patients with a complete tumour response undergoing a wait-and-see approach after chemoradiation treatment were followed using a combination of standard MRI and DWI. The morphology of the rectal wall was studied, both shortly after treatment and during long-term follow-up. Complete responders either show a completely normalised rectal wall or postradiation fibrosis. In case of fibrosis, the shape and volume of the fibrosis is related to the aspect of the tumour before treatment. In case of a sustained complete response, the pattern of the normalised wall or fibrosis remains consistent during long-term follow-up.

Chapter 8 describes a study in which the use of standard MRI, DWI and image fusion of standard MRI and DWI were compared for the diagnosis of locally recurrent rectal cancer during follow-up after primary surgical treatment. It showed that standard MRI can accurately detect a local tumour recurrence. Addition of DWI or fusion images does not significantly improve its performance, but may be useful to decrease the number of false positive assessments and improve the agreement between different observers. 


\section{Samenvatting}

Het doel van dit proefschrift was te onderzoeken of en hoe diffusie-gewogen MRI en gadofosveset contrast-versterkte MRI kunnen bijdragen aan de klinische selectie van patiënten voor orgaans-sparende behandelingen. Dit proefschrift is opgedeeld in drie delen: tumor respons evaluatie, lymfeklierstadiëring en follow-up.

\section{Deel I-Tumor respons}

Hoofdstuk 2 beschrijft een multicentrische studie waarin de diagnostische waarde van diffusie-gewogen MRI als toevoeging aan standaard T2-gewogen MRI werd onderzocht voor het identificeren van patiënten met een complete tumor respons na chemoradiatie therapie. Voor lezers met minder ervaring bleek de diagnostische accuratesse significant te verbeteren na toevoeging van DWI. Met name de sensitiviteit voor het detecteren van de complete responders verbeterde met 16 $25 \%$, wat aangeeft dat de mate waarin de respons na behandeling wordt onderschat aanzienlijk kan worden gereduceerd in vergelijking met standaard beeldvorming.

In de studie in hoofdstuk 3 onderzochten we volumetrische en ADC metingen met behulp van DWI voor het analyseren van tumor respons en vergeleken dit met tumor volumetrie middels T2-gewogen MRI. Na chemoradiatie konden we met behulp van tumor volumes gebaseerd op hoog signaal gebieden op DWI accuraat de patiënten met een complete tumor respons identificeren. Deze techniek bleek significant beter dan conventionele tumor volumetrie. Tumor volume reductie ratio's berekend tussen de pre- en post-chemoradiatie beelden van zowel standaard MRI als DWI bleken even accuraat, hoewel deze methoden evident meer tijd kosten dan enkel het beoordelen van post-chemoradiatie DWI. ADC metingen, alsmede pre-chemoradiatie volumetrie waren niet bruikbaar voor respons evaluatie.

In hoofdstuk 4 onderzochten we het effect van verschillende 'Regions of Interest' typen en grootten op het meten van tumor ADC waarden. De ROI-keuze bleek significant van invloed op de tumor ADC waarden, alsmede op de interobserver variabiliteit. ADC metingen gemiddeld voor het gehele tumor volume waren beter reproduceerbaar dan metingen verkregen van een enkele tumor plak of een klein monster van de tumor. Metingen verricht voor chemoradiatie therapie bleken beter reproduceerbaar dan metingen verricht na behandeling.

\section{Deel II - Lymfeklieren}


In hoofdstuk 5 analyseerden we het gebruik van DWI voor het beoordelen van lymfeklieren na chemoradiatie behandeling. Visuele beoordeling van DWI verbetert het totaal aantal gedetecteerde lymfeklieren ten opzichte van standaard MRI, maar is niet van nut voor het karakteriseren van lymfeklieren, daar zowel benigne als metastatische klieren dezelfde signaalkarakteristieken laten zien. ADC waarden zijn wel significant verschillend tussen benigne en metastatische klieren, maar het toevoegen van $A D C$ aan standaard T2-gewogen MRI resulteert niet in een significante verbetering in de diagnostische accuratesse voor lymfeklierstadiëring.

Hoofdstuk 6 beschrijft het gebruik van een nieuw contrastmiddel, gadofosvesettrisodium, voor het beoordelen van lymfeklieren. Het gebruik van gadofosveset resulteert in een significant verbeterde diagnostische betrouwbaarheid in vergelijking met standaard MRI. Omdat gezonde klieren gadofosveset opnemen, laten deze klieren een sterke toename in signaal zien. Metastasen daarentegen blijven donker. Het signaal van de klieren in combinatie met het 'reliëf effect' zorgt voor een hoge sensitiviteit en specificiteit voor het differentiëren tussen benigne en metastatische klieren. Het effect ten opzichte van standaard MRI is met name evident voor de primaire stadiëring van lymfeklieren. Na chemoradiatie is het gebruik van enkel standaard MRI al relatief accuraat.

\section{Deel III - Follow-up}

In hoofdstuk 7 volgden we patiënten met een complete tumor respons tijdens een 'wait-and-see' beleid middels een combinatie van standaard MRI en DWI. De morfologie van de darmwand werd bestudeerd zowel kort als lang na behandeling. De complete responders toonden hierbij een volledig genormaliseerde darmwand of fibrose. In het geval van fibrose bleek de vorm en het volume van de fibrose gerelateerd aan het aspect van de primaire tumor voor aanvang van behandeling. Wanneer er sprake was van een blijvende complete respons bleef het patroon van de genormaliseerde wand of fibrose constant tijdens de verdere follow-up.

In hoofdstuk 8 vergeleken we standaard MRI, DWI en gefuseerde MRI + DWI beelden voor het diagnosticeren van een lokaal recidief rectumcarcinoom tijdens de klinische follow-up na primaire chirurgische behandeling. Met standaard MRI kan een lokaal recidief betrouwbaar worden vastgesteld. Toevoeging van DWI of fusie plaatjes zorgt niet voor een significant verbeterde diagnostische betrouwbaarheid, maar kan wel van nut zijn voor het verminderen van het aantal fout-positieve bevindingen en om de overeenstemming tussen verschillende radiologen te verbeteren. 


\section{Dankbetuigingen}

Zoals de zorg voor rectumcarcinoom patiënten sterk afhankelijk is van de kwaliteit van het 'multidisciplinaire team', zo is zeker ook bij het totstandkomen van een proefschrift een goed team onontbeerlijk. Het is onmogelijk om iedereen die direct of indirect - een voor mij waardevolle bijdrage heeft geleverd hier bij naam te noemen (zonder mijn dankwoord tot een vuistdikke bijlage te laten uitdijen), maar een persoonlijk woord van dank wil ik toch graag richten tot de volgende personen:

Prof. dr. Regina Beets-Tan. Beste Regina, meteen in het voor mij nog prille begin, toen ik nog als student stage liep op de afdeling radiologie, heb je me weten te 'besmetten' met het virus dat wetenschap heet. Jouw ongekende passie voor het onderzoek, je vermogen om overal nieuwe kansen en uitdagingen in te zien, je teamspirit en het feit dat boven alles de zorg voor 'de patiënt' altijd op de eerste plaats blijft staan, zijn voor mij een bijzondere inspiratie geweest. Ik heb de afgelopen jaren ontzettend veel van je geleerd, ben je zeer dankbaar voor de vele unieke kansen die je me hebt geboden en waardeer enorm het gevoel dat ik altijd bij je aan kan kloppen. Ik had me geen betere mentor kunnen wensen en kijk uit naar nog een lange en vruchtbare samenwerking.

Dr. Beets. Beste Geerard, als 'nuchtere' tweede helft van mijn promotieteam ben jij tijdens mijn promotietraject bijzonder belangrijk geweest. Jouw kritische en nuchtere blik heeft me vaak geholpen dingen in het juiste perspectief te plaatsen. Ik heb ontzettend bewondering voor de manier waarop je jouw visie van het toch wel omstreden 'wait-and-see' beleid samen met ons hebt weten te realiseren tot niet alleen een succesvolle studie, maar veel belangrijker nog: tot een manier om patiënten met endeldarmkanker een nieuw soort toekomst te bieden.

Prof. dr. Jos van Engelshoven: het is mij een eer dat $u$ de rol van voorzitter van mijn beoordelingscommissie op zich heeft willen nemen. Veel dank voor de uitermate vriendelijke manier waarop $u$ altijd interesse heeft getoond in de vorderingen van mijn proefschrift. De overige leden van de beoordelingscommissie, Prof. dr. Karin Kaustermans, dr. Harm Rutten, Prof. dr. Felix Mottagy en Prof. dr. Klaas Nicolay, alsmede de leden van mijn oppositiecommissie, wil ik eveneens zeer hartelijk bedanken voor de kritische beoordeling van mijn proefschrift.

$\operatorname{Dr}$ (die -s mag er alvast wel af vind ik) M. Maas. Monique / Mo / Monkie - en alle andere nicknames waar ik je de afgelopen jaren mee om de oren heb geslingerd - ik ben ontzettend trots op wat wij de afgelopen jaren samen hebben weten te 
bereiken en de vriendschap die hieruit als bij-effect heeft mogen voortvloeien. Ik denk dat de mate van 'jut-en-jul' die ons regelmatig wordt toebedicht een directe reflectie is van hoe wij hebben samengewerkt. Veel dank voor je loyale partnerschap, je research-enthousiasme, je kritische (statistische) blik, maar zeker ook je oeverloze gekwetter en onverscholen LINGO talent.

De overige leden van het 'rectum-team': Sanne, Max, Elleke, Ewelina, Luc en Milou. Sanne en Max, mijn onderzoekstijd is werkelijk met een kickstart begonnen, wat nooit mogelijk zou zijn geweest als jullie de weg niet al zo mooi geplaveid hadden, waarvoor ik jullie zeer erkentelijk ben. Sanne, ik ga jou vast nog tegenkomen in de kliniek als ongetwijfeld bevlogen chirurg. Max en Elleke, het is me een genoegen om nu ook als klinische collega's met jullie te mogen samenwerken! Ewelina, heel veel geluk samen met jullie eerste kindje, we zien je graag snel weer terug in Maastricht. Luc en Milou: de eer is nu aan jullie! Ik ben er van overtuigd dat we nog een plezierige verdere samenwerking tegemoet gaan.

Ook absoluut onmisbaar binnen ons team zijn de leden van de abdomengroep radiologie, waarbij mijn speciale dank uitgaat naar Drs. Frans Bakers en Dr. Vincent Cappendijk (ondertussen werkzaam in het Jeroen Bosch Ziekenhuis te Den Bosch). Talloze uren hebben jullie besteed aan het geduldig met ons naar kliertjes, diffusieplaatjes en andere rectum-eigenaardigheden te turen. Zonder jullie bijdrage had een groot deel van dit proefschrift niet tot stand kunnen komen.

Mijn overige klinische collega's van de afdeling radiologie: na het afronden van mijn onderzoeksperiode in de kelder ben ik een paar verdiepingen hoger absoluut in een warm bad terecht gekomen. Mijn speciale dank aan onze opleider, Prof. dr. Michiel de Haan, en ons afdelingshoofd, Prof. dr. Joachim Wildberger, mede ook dat zij beide zo ruimdenkend zijn geweest om mij ook tijdens mijn opleiding het realiseren van mijn onderzoeksambities te laten voortzetten.

A warm thanks goes out to Dr. Luis Curvo Semedo: Luis, I really enjoyed 'those days you spent in Maastricht', as much as I recently enjoyed the few I got to spent in Portugal for your thesis defence. It has been a joy to work with you and I am very proud of the papers we have written together.

Ik heb tijdens mijn promotie periode het voorrecht gehad met ontzettend veel inspirerende personen - zowel in Nederland als internationaal - te mogen samenwerken. Hierbij wil ik in het bijzonder bedanken Dr. Vincent Vandecaveye uit het UZ Leuven, mijn toekomstige (tijdelijke) opleider Dr. Roy Vliegen uit het Atrium 
medisch centrum en Prof. dr. Jaap Stoker uit het AMC Amsterdam. A special thanks to Dr. Brunella Barbaro and Prof. Vincenzo Valentini from Rome Catholic University, to Sofia Gourtsoyiannis (with whom I took the first step towards investigating diffusion MRI) and Dr. Nickolas Papanikolaou from Greece and Prof. dr. Celso Matos from Brussels.

Ik ben veel dank verschuldigd aan alle MRI laboranten en al het ondersteunend personeel dat zich de afgelopen jaren heeft ingezet voor ons onderzoek. Hierbij mijn speciale dank aan Sylvia Gijzen en Guus van der Groen, voor het onvermoeibaar inplannen (en omplannen) van MRI onderzoeken, Frans Kusters voor het inlezen en opvragen van talloze onderzoeken, alsmede Henk Schoenmakers en later Roland Kersemakers voor hun bezielende leiding aan het MRI team en het creëren van ruimte om ons onderzoek - en aldus ook de zorg voor de rectumcarcinoompatiënten - verder te laten groeien.

Jan Verwoerd (Philips Healthcare). Jan, jouw technische MRI ondersteuning is voor ons onderzoek van onschatbare waarde geweest. Ontelbare keren heb je ons uit de brand geholpen als we weer eens tegen een exotisch (of in alle eerlijkheid minder exotisch) MRI probleem aanliepen. Ik heb ontzettend veel van je geleerd en hoop dat we onze plezierige samenwerking nog lang mogen voortzetten. Mijn eerste vlieg-ervaring was er een om nooit te vergeten, waarvoor ook veel dank aan je lieve vrouw Elly.

Een blijk van dank aan alle collega-onderzoekers die onze periode in de kelder mede hebben opgefleurd. Eline en Walter voor hun leiding aan de onderzoeksgroep. En in het bijzonder dank aan Marc en Jos (zij weten alles van computers!), in de eerste plaats voor het geduld dat zij steeds hebben weten op te brengen als we hen weer kwamen stalken met een computer (of willekeurig welk ander electronica apparaat) probleem, maar zeker niet in de laatste plaats voor de gezelligheid.

Fons Kessels, heel veel dank voor je hulp bij onze statistische vraagstukken en je regelmatige gezellige bezoekjes aan de kelder. Ine Kengen, bedankt voor al je hulp bij het opmaken van van mijn proefschrift. Tim Leiner, jij liet me voor het eerst kennis maken met de wetenschap, waarvoor mijn bijzondere dank!

Bij het totstandkomen van dit proefschrift zijn vele partijen binnen en buiten het MUMC+ betrokken geweest. Ik ben hierbij allereerst dank verschuldigd aan de medewerkers van GROW School for Oncology and Developmental Biology. Verder hebben wij in het MUMC nauw samengewerkt, niet alleen met de afdelingen 
radiologie en chirurgie, maar zeker ook met de afdelingen pathologie, medische oncologie, maag-darm-leverziekten en radiotherapie (Maastro Clinic), waarvoor ik de betrokken personen graag hartelijk wil bedanken. Eveneens mijn dank voor de prettige samenwerking in de regio met de betrokkenen van het Atrium Medisch Centrum te Heerlen, het Orbis Medical Concern te Sittard en het Laurentius Ziekenhuis te Roermond.

Lieve familie, lieve vrienden. Mijn plezier in mijn werk is uiteraard ook een directe reflectie van mijn ontzettende plezier buiten het werk. Papa en mama, heel veel dank voor de goede basis die jullie mij hebben meegegeven. Allerliefste oma, ik draag dit boek op aan $u$, ik vind het ontzettend jammer dat het $u$ niet gaat lukken om bij de verdediging aanwezig te kunnen zijn. Naomi, Tirza, Sira en Thomas, ik ben heel trots op ons broeder-zusters verbond! Toon en Monique, heel veel dank voor de liefdevolle manier waarop jullie mij ook in jullie familie hebben verwelkomd. Ellen, paranimf, naast Roel ben jij al sinds jaar en dag mijn 'wederhelft', bedankt dat je ook vandaag weer naast me wilt staan. Barbe, ik reken ook op jou als soort van 'derde' paranimf! Hoewel ik niet iederaan zo vaak kan zien als ik graag zou willen en ook niet iedereen hier bij naam kan noemen, prijs ik mezelf zeer gelukkig met mijn fijne groep vrienden in Maastricht, alsmede in Breda en verder verspreid door het land. Bedankt voor de interesse die jullie hebben getoond in mijn promotie en vooral voor jullie warme vriendschap.

Lieve Roel, ik denk niet dat ik woorden nodig heb om uit te drukken wat jij de afgelopen jaren voor mij hebt betekend. En dan heb ik het nog niet eens over je onvolwaardig geprezen directe bijdrage aan mijn proefschrift: het is een bijzonder mooie tabel geworden;)

Keep from you!

Tot slot, maar zeker niet in de laatste plaats, wil ik graag de vele patiënten in Nederland bedanken die in deze moeilijke periode van hun leven de moed en de bereidheid hebben getoond om te participeren in ons onderzoek. 


\section{Curriculum vitae}

Doenja Marina Johanna Lambregts was born on November 4th, 1983 in Breda, The Netherlands. In 2002 she graduated Cum Laude from the Onze Lieve Vrouwelyceum in Breda, The Netherlands. In the same year she moved to Maastricht, where she started her medical training at the Faculty of Medicine at the University of Maastricht. She attended her elective and scientific internships at the radiology department of MUMC+ under supervision of Prof. dr. S.F. Robben en Dr. T. Leiner. During this period, she also made her first

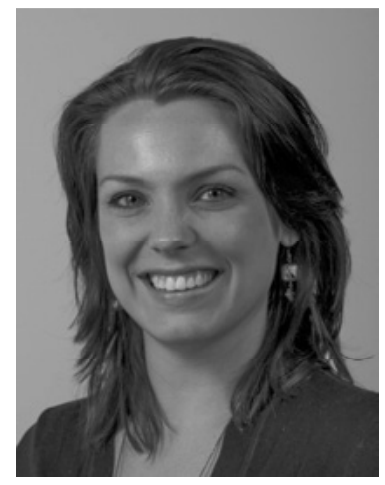
encounter with the research team led by Prof. dr. R.G.H. Beets-Tan and Dr. G.L. Beets. As a preparation for a future PhD position she decided to become involved (alongside her ongoing interships) in their research programme investigating the diagnosis and treatment of rectal cancer patients. From August 2008, immediately after obtaining her medical doctor degree, she officially started working as a PhD student under supervision of Prof. dr. Beets-Tan and Dr. Beets. The main goal of her PhD project was to study the use of a new contrast agent for the assessment of lymph node metastases in rectal cancer and to investigate a new technique, diffusion-weighted MRI, for treatment response evaluation after chemoradiotherapy. The results of these studies have resulted in this thesis. Her research was awarded the 'Dutch Radiology Society Meeting award' in 2010 (for which she was again nominated in 2011) and the 'Best Scientific Paper Presentation Award' at the European Congress of Radiology in both 2010 and 2011.

In April 2011, Doenja Lambregts started her radiology residency at the MUMC+ (Prof. dr. J. Wildberger, Prof. dr. M.W. de Haan). Furthermore, she remains involved in the rectal cancer research programme led by Prof. Dr. Beets-Tan and Dr. Beets and is currently co-supervising the present PhD students. 


\section{List of publications}

\section{This thesis}

1. Lambregts DMJ, Vandecaveye $\mathrm{V}$, Barbaro B, Bakers FCH, Lambrecht $M$, Maas $M$, Haustermans K, Valentini V, Beets GL, Beets-Tan RGH. Diffusion-weighted MRI for selection of complete responders after chemoradiation for locally advanced rectal cancer: a multicenter study. Ann Surg Oncol 2011;18(8):2224-31

2. Curvo-Semedo L, Lambregts DMJ, Maas M, Thywissen $T$, Mehsen RT, Lammering G, Beets GL, Caseiro-Alves F, Beets-Tan RGH. Rectal cancer: Assessment of a complete response to preoperative combined radiation therapy with chemotherapy: comparison between conventional MR volumetry and diffusion-weighted MR imaging. Radiology 2011;260(3):734-43

3. Lambregts DMJ, Beets GL, Maas M, Curvo-Semedo L, Thywissen T, Beets-Tan $\mathrm{RGH}$. Tumour ADC measurements for evaluation of treatment response in rectal cancer: influence of Interobserver variability and measurement methods. Eur Radiol 2011;21(12):2567-74

4. Lambregts DMJ, Maas M, Riedl RG, Bakers FCH, Verwoerd JL, Kessels AGH, Lammering G, Boetes C, Beets GL, Beets-Tan RGH. Value of ADC measurements for nodal staging after chemoradiation in locally advanced rectal cancer - a per lesion validation study. Eur Radiol 2011;21(2):265-273

5. Lambregts DMJ, Beets GL, Maas M, Kessels AGH, Bakers FCH, Cappendijk VC, Engelen SME, Lahaye MJ, de Bruïne AP, Lammering G, Leiner T, Verwoerd JL, Wildberger JE, Beets-Tan RGH. Accuracy of gadofosveset-enhanced MRI for nodal staging and restaging in rectal cancer. Ann Surg 2011;253(3):539-545

6. Lambregts DMJ, Maas M, Bakers FCH, Cappendijk VC, Lammering G, Beets GL, Beets-Tan RGH. Long-term follow-up features on rectal MRI during a wait-andsee approach after a clinical complete response in rectal cancer patients treated with chemoradiotherapy. Dis Colon Rectum 2011;54(12):1521-28

7. Lambregts DMJ, Cappendijk VC, Maas M, Beets GL, Beets-Tan RGH. Value of $M R I$ and diffusion-weighted MRI for the diagnosis of locally recurrent rectal cancer. Eur Radiol 2011;21(6):1250-58

\section{Other}

8. Lambregts DMJ, Maas M, Cappendijk VC, Prompers LM, Mottaghy FM, Beets GL, Beets-Tan RGH. Whole body diffusion-weighted magnetic resonance imaging: current evidence in oncology and potential role in colorectal cancer staging. Eur J Cancer 2011;47(14):2107-16 
9. Lambregts DMJ, Heijnen LA, Maas M, Rutten IJG, Martens MH, Backes WH, Riedl RG, Bakers GCH, Cappendijk VC, Beets GL, Beets-Tan RGH. Gadofosvesetenhanced MRI for the assessment of rectal cancer lymph nodes: predictive criteria. Abdom Imaging 2012 [Sep 18, ePub ahead of print]

10. Curvo-Semedo L, Lambregts DMJ, Maas M, Beets GL, Caseiro-Alves F, Beets-Tan RGH. Diffusion-weighted MRI in rectal cancer: apparent diffusion coefficient as a potential noninvasive marker of tumour aggressiveness. J Magn Reson Imaging 2012;35(6):1365-71

11. Maas M, Beets-Tan RGH, Lambregts DMJ, Lammering G, Nelemans PJ, Engelen SM, van Dam RM, Jansen RL, Sosef M, Leijtens JW, Hulsewé KW, Buijsen J, Beets GL. Wait-and-see policy for clinical complete responders after chemoradiation for rectal cancer. J Clin Oncol 2011;39(35):4633-40

12. Maas M, Rutten IJG, Nelemans P, Lambregts DMJ, Cappendijk VC, Beets GL, Beets-Tan RGH. What is the most accurate whole-body imaging modality for assessment of local and distant recurrent disease in colorectal cancer? A metaanalysis: imaging for recurrent colorectal cancer. Eur J Nucl Med Mol Imaging 2011;38(8):1560-71

13. Maas M, Lambregts DMJ, Lahaye MJ, Beets GL, Backes W, Vliegen RFA, Osingade Jong M, Wildberger JE, Beets-Tan RGH. Accuracy of 3.0 Tesla MRI compared with 1.5 Tesla for T staging of rectal cancer. Abdom Imaging 2012;37(3):475-81

14. Kluza E, Rozenboom ED, Maas M, Martens M, Lambregts DMJ, Slenter J, Beets GL, Beets-Tan RGH. T2 weighted signal intensity evolution may predict pathological complete response after treatment for rectal cancer. Eur Radiol 2013;23(1):253-61

15. Lahaye MJ, Beets GL, Engelen SME, Voth M, Leiner T, Lambregts DMJ, Beets-Tan RGH. Gadofosveset Trisodium (Vasovist ) enhanced MR lymph node detection: initial observations. The Open Magnetic Resonance Journal 2009;2:1-5

\section{Book chapters}

1. Lambregts DMJ, Maas M, Beets-Tan RGH. MRI of the Rectum, in Stoker J (ed): MRI of the Gastrointestinal Tract. Springer; 2010: p205-228

2. Lambregts DMJ, Beets-Tan RGH. How can we identify local relapse (Q\&As on imaging), in Valentini V (ed): Multidisciplinary Management of Rectal Cancer. Springer; 2012: p95-102

3. Maas M, Lambregts DMJ, Beets-Tan RGH. MR Imaging of the rectum, $3 T$ vs $1.5 T$, in Kamel I (ed): Body MR imaging at 3.0 Tesla. Cambridge University Press, 2011: p150-163 